\title{
SUBSONIC AND SUPERSONIC JETS AND SUPERSONIC SUPPRESSOR CHARACTERISTICS
}

BY $H, T$, NAGAMATSU AND R,E, SHEER, JR.

Prepared under Contract No. NASW-1784 by

GENERAL ELECTRIC RESEARCH AND DEVELOPMENT CENTER

Schenectady, New York

\section{Prepared For:}

National Aeronautics and Space Administration

Lewis Research Center

21000 Brookpark Road

Cleveland, Ohio

November 10,1972 


\section{FOREWORD}

This report was prepared under contract No. NASW-1784 under the initial management of Mr. I.R. Schwartz, Chief, Fluid Mechanics Branch, NASA Headquarters, Washington, D.C., the management was taken over by Dr. W.H. Roudebush, also at NASA Headquarters, in March 1971 and the current project manager is Mr. E. Krejsa, NASA - Lewis Research Center. The work was conducted at the Mechanical Engineering Laboratory, General Electric Research and Development Center in Schenectady, New York. 


\section{SUMMARY}

Convergent and parallel flow nozzles were used with room temperature air to investigate the flow and acoustic characteristics over a Mach number range of 0.6 to 1.5. For subsonic jet Mach numbers the core region extended over. 4 to 5 diameters, depending on the turbulence level, and the velocity decreased as $x^{-1}$ in the fully developed subsonic turbulent flow. For supersonic jet Mach numbers the sonic location on the axis increased as $M_{j}^{2}$, and the velocity decayed in the subsonic region as $x^{-1}$. Peak impact pressure fluctuations on the axis occurred at 9 diameters for subsonic jets, and the pressure fluctuations decreased as $x^{-1.74}$ for the turbulent region. For supersonic jets the peak pressure fluctuations occurred just ahead of the sonic point.

For subsonic jets the maximum sound pressure levels occurred at 19.1 from the axis and the sound pressure level decreased monotonically with increasing angle. But for supersonic jets the sound pressure was nearly constant over most of angular positions. The power spectra for subsonic jets were similar with the peak occurring at $4 \mathrm{KHz}$ and for a Mach 1.4 jet the peak occurred at 5 $\mathrm{KHz}$. Near field sound pressure level distributions were quite similar for subsonic jets, but for supersonic jets the distributions were quite different. Overall sound power levels were compared with the subsonic theory of Lighthill and supersonic theory of Nagamatsu and Horvay. The exponents $\alpha$ and $\beta$ in this theory were evaluated for parallel and convergent nozzles as functions of the jet Mach number.

Mean and fluctuating velocity contours were determined for a convergent nozzle with the impact and piezoelectric impact pressure gage probes for Mach numbers of 0.6 to 1.4. For subsonic Mach numbers the peak pressure fluctuations occurred in a toroidal region located 4 to 6 diameters from the nozzle. But for supersonic Mach numbers the peak impact pressure fluctuations occurred close to the sonic velocity contour. Contours for the constant rms static pressure fluctuations were determined at Mach numbers of 0.6 to 1.4 , and for subsonic Mach numbers were similar with the peak fluctuations occurring close to $r / r_{0}=1.0$ near the nozzle exit. For supersonic Mach numbers the contours of static pressure fluctuations were quite different than the contours for subsonic Mach numbers. Hot-wire and laser doppler velocimeter methods were used to calibrate the piezoelectric impact and static pressure probes.

A supersonic suppressor of 191 tubes and shrouds was investigated at Mach numbers of 1.4 and 0.7 . The primary jet Mach number was drastically decreased, and the rms impact and static pressure fluctuations on the axis were also reduced from the values existing 
for a single nozzle. For a. Mach 1.4 jet the noise level was reduced $15.3 \mathrm{db}$ with 191 tubes and $20.5 \mathrm{db}$ with the shrouds. And the corresponding reductions for a Mach 0.7 jet were $4.5 \mathrm{db}$ and $7.8 \mathrm{db}$ respectively. A single. long. shroud and six rods indicated large reductions in the jet velocity at the shroud exit with and without induced flow. For a Mach 1.4 jet the noise level was reduced $14.2 \mathrm{db}$ with induced flow and $4.5 \mathrm{db}$ without induced flow. 
SUMMARY

$\mathrm{v}$

TABLE OF CONTENTS

vii

LIST OF FIGURES

$\mathrm{xi}$

LIST OF SYMBOLS

$\mathrm{xV}$

1.0 INTRODUCTION

2.0 EXPERIMENTAL FACILITIES AND PROCEDURE

2.1 Test Facilities

2.2 Procedure

3.0 INSTRUMENTATION AND CALIBRATION 9

3.1 Instrumentation

3.2 Calibration Shock Tube

3.3 Laser Doppler Velocimeter 10

3.4 Piezoelectric Impact and Static Pressure. 11 Probes

4.0 ANALYSIS OF PIEZOELECTRIC IMPACT AND STATIC PRESSURE FLUCTUATIONS AND TURBULENT VELOCITY FLUCTUATIONS

4.1 Analysis of Piezoelectric Impact Pressure Fluctuations

4.2 Analysis of Piezoelectric Static Pressure Fluctuations

5.1 Axial Distributions of Mean Velocity 18

5.2 Axial Piezoelectric Impact Pressure 19 Fluctuations 
5.3 Axial Piezoelectric Static Pressure

Fluctuations

5.4 Contours of Mach Number, Piezoelectric

Impact and Static Pressure Fluctuations

6.1 Flow and Acoustic Characteristics of

Subsonic and Supersonic Jets

6.2 Jet Noise Theories

6.2.1 Subsonic Jet Noise Theory of Lighthill

6.2.2 Supersonic Jet Noise Theory of Nagamatsu and Horvay

6.3 Near-Field Acoustic Characteristics

6.3.1 Near-Field Pressure Fluctuations

6.3.2 Distribution of Acoustic Power

6. 4 Sound Pressure Level Distribution

6.5. Sound Power Spectra and Overall Sound Power Level

6.5.1 Sound Power Spectra

6.5.2 Overall Sound Power Level

6. 6 Sound Pressure Level Spectra as Function of

Angular Position and Mach Number

7.0 MULTIPLE TUBE AND. SINGLE AND MULTIPLE SHROUD

SUPPRESSORS FOR SUPERSONIC JETS

7.1 Single Shroud with Six Rod Suppressor 35

7.2 191 Tubes and 191 Shrouds Suppressor 36

7.2.1 Flow Characteristics 
Page Number.

7.2.2 Thrust Loss

7.3 191 Tubes and 191 Shrouds Suppressor

for Mach 0.7 Jet

7.3.1 Flow Characteristics

7.3.2 Acoustic Characteristics

8.0 CONCLUSIONS

REFERENCES

TABLE :

FIGURES

54 
Figure 1

Figure 2

Figure 3

Figure 4

Figure 5

Figure 6

Figure 7

Figure $8 a$

Figure $8 b$

Figure 9 a

Figure $9 b$

Figure 10a

Figure $10 \mathrm{~b}$

Figure $11 \mathrm{a}$
Two Inch and 6.15 Inch Diameter Flow and Acoustic Facility.

One Inch Convergent Nozzle and Flow Field Survey Equipment.

IDV Setup at General Electric Research and Development Center.

Probes for Jet Exhaust Surveys.

Eight Inch Calịbration Shock Tube.

Experimental Arrangement for Jet Turbulence Measurements with Laser Doppler Velocimeter.

Piezoelectric Quartz Pressure Gage Output as a Function of Pressure Jump Across Shock Waves.

Velocity Ratio on Axis and Axial Turbulence Level with Hot Wire, LDV, and Piezoelectric Impact Pressure Probe for $3 / 4$ Inch Nozzle, $M_{j}=0.3$.

Velocity Ratio on Axis and Axial Turbulence Level with Piezoelectric Impact Pressure Probe and LDV for $1 / 2$ Inch Nozzle, $M_{j}=1.2$.

Axial RMS Static Pressure Fluctuations Determined with Piezoelectric Static Pressure Probe and from Hot-Wire Turbulence Measurements for $3 / 4$ Inch Nozzle, $M_{j}=0.3$.

Axial RMS Static Pressure Fluctuations Determined with Piezoelectric Static Pressure Probe and LDV Turbulence Measurements for $3 / 4$ Inch Nozzle, $M_{j}=$
0.6 .

Velocity Ratio on Jet Axis for Two Inch Diameter Convergent Nozzle.

Flow Mach Number Ratio on Jet Axis for One Inch Diameter Convergent Nozzle.

Variation of Impact Pressure Fluctuations on Jet Axis with Distance from Jet Exit for Two Inch Diameter Convergent Nozzle. 
Figure $11 b$

Figure 12

Figure $13 a$

Figure 13b

Figure $13 c$

Figure 14

Figure 15a

Figure $15 b$

Figure 16

Figure 17

Figure 18

Figure 19a

Figure $19 \mathrm{~b}$

Figure $20 a$
Variation of Piezoelectric Pressure Fluctuations on Jet Axis with Distance from Jet Exit for one Inch Diameter Convergent Nozzle.

Shadowgraph Photographs of. Flow from a Convergent Nozzle at Mach Numbers of 1.0 and 1.4 .

Axial Static Pressure Fluctuations for Two Inch Convergent Nozzle.

Variation of Piezoelectric Static Pressure Fluctuations on Jet Axis with Distance from Jet Exit for One Inch Diameter Convergent Nozzle.

Variation of Piezoelectric Static Pressure Fluctuations on Jet Axis as a Function of Local Mach Number for One Inch Diameter Convergent Nozzle.

Constant Mach Number Contours in the Flow Field from a One Inch Convergent Nozzle, $\mathrm{M}_{j}=1.0$ and
1.4 .

Constant Piezoelectric Impact and Static Pressure Fluctuation Contours in the Flow Field from a one Inch Convergent Nozzle, $\mathrm{M}_{\mathrm{j}}=1.0$.

Constant Piezoelectric Impact and Static Pressure Fluctuation Contours in the Flow Field from a one Inch Convergent Nozzle, $\mathrm{M}_{j}=1.4$.

Comparison of Subsonic and Supersonic Jet Aerodynamic and Acoustic Characteristics.

Parallel Flow Supersonic Jet Expanded to Ambient Pressure.

Jet Core Length, and Supersonic Length as a Function of Jet Mach Numbers.

Acoustic Power Generated Per Unit Length of the Jet.

Acoustic Power Per Unit Length of Jet. Obtained with Microphone Traverse Parallel to Jet Axis at Two Nozzle Diameters from Nozzle Periphery.

Acoustic Power Generated Per Unit Length of the Jet for Jet Mach Number of 1.0 . 
Figure $20 \mathrm{~b}$

Figure $21 a$

Figure $21 b$

Figure 22

Figure 23

Figure 24

Figure 25

Figure 26

Figure 27a

Figure 27b

Figure 28

Figure 29a

Figure 29b

Figure $29 c$

Figure 30
Acoustic Power Generated Per Unit Length of the Jet for Jet Mach Number of 1.5.

Overall Sound Power Level in db Per Unit Slug Mass as a Function of Jet Mach Number.

Overall Sound Power Level in $d b$ Per: Unit Slug Mass as a Function of Jet Velocity.

Sound Pressure Level Variation Parallel to Jet Axis at 2 and 4 Diameters from Nozzle Exit.

Overall Sound Pressure Level as a Function of Angular Position from Jet Axis for Different Jet Mach Numbers.

Comparison of Sound Power Spectra for Plain Convergent Jet at Various Mach Numbers.

Overall Sound Power Level in Watts for Constant Mass Flow as Function of Jet Velocity.

Values of Exponents $\alpha$ and $\beta$ of of Nagamatsu-Horvay Supersonic Jet Noise Theory as a Function of Jet Mach Number for Parallel Flow and Convergent Nozzle with $\mathrm{T}_{\mathrm{t}_{\mathrm{j}}}=520^{\circ} \mathrm{R}$.

Sound Pressure Level vs. Frequency at 8 Angular Positions from Jet Axis for a 2 Inch Diameter Convergent Nozzle, $M_{j}=1.0$.

Sound Pressure Level vs. Frequency at 8 Angular Positions from Jet Axis for 2 Inch Diameter Convergent Nozzle, $\mathrm{M}_{j}=1.4$.

Sketch of Nozzle, Shroud, and Rods.

Axial Mach Number Distribution for 2 Inch Diameter Convergent Nozzle with and without Shroud and Rods.

Overall Sound Pressure Level as Function of Angular Position from Jet Axis for 2 Inch Diameter Convergent Nozzle with and without Shroud and Rods.

Sound Power Level Spectra for 2 Inch Diameter Convergent Nozzle with and without Shroud and Rods.

Sketch of Multitube Suppressor and Shrouds. xiii 
Figure 31 a

Figure $31 b$

Figure 3lc

Figure $31 d$

Figure $3 l e$

Figure $3 l f$

Figure $32 \mathrm{a}$

Figure $32 \mathrm{~b}$

Figure 32c

Figure $32 d$

Figure $32 e$
Mach Number Profile Across Exits of Multitubes and Multishrouds.

Axial Mach Number Variation for Multitubes with and without Shrouds.

Axial Impact Pressure Fluctuations for Multitubes with and without Shrouds.

Overall Sound Pressure Level as a Function of Angular Position from Jet.Axis for Multitubes with and without shrouds.

Sound Power. Level Spectra for Multitubes with and without Shrouds.

Variation of Overall Sound Power Level Reduction and Percentage Thrust Loss for 191 Tubes with 191 Shroud Length.

Axial Mach Number Distribution for Multitubes with and without Shrouds, $M_{j}=0.7$.

Axial Impact Pressure Fluctuations for Multitubes with and without Shrouds, $\mathrm{M}_{j}=0.7$.

Axial Static Pressure Fluctuations for Multitubes with and without Shrouds, $M_{j}=0.7$.

Overall Sound Pressure Level as a Function of Angular Position from Jet Axis for Multitubes with and without Shrouds, $M_{j}=0.7$.

Sound Power. Level Spectra for Multitubes with and without Shrouds, $M_{j}=0.7$. 


\section{LIST OF. SYMBOLS}

A

C

ca

$c_{j}$

$\mathrm{C}_{\mathrm{f}}$

$\mathrm{C}_{\mathrm{i}}$

D

I

$\ell_{\mathrm{C}}$

$\ell_{s}$

$\mathrm{I}_{\mathrm{s}}$

m

M

$\mathrm{M}_{j}$

$\mathrm{p}_{\mathrm{a}}$

po

po

$\overline{\mathrm{p}}$

$\left\langle\mathrm{p}^{\prime}\right\rangle$

$\tilde{\mathrm{p}}$

$\mathrm{p}_{\mathrm{f}}$

$\mathrm{p}_{\mathrm{T}}$

$\mathrm{P}_{\mathrm{T}}^{\prime}$

cross sectional area

velocity of sound

velocity of sound in ambient air

velocity of sound in jet

skin friction

incompressible turbulent skin friction

nozzle exit diameter

acoustic intensity

jet core length

supersonic length

nondimensionalized supersonic length, $\ell_{s} / D$

mass flow of jet

Mach number

jet Mach number

ambient pressure

total pressure

impact pressure after normal shock

mean static pressure

pressure fluctuation caused by turbulence

pressure fluctuation due to sound field

static pressure fluctuation

total pressure along streamline

total pressure fluctuation 


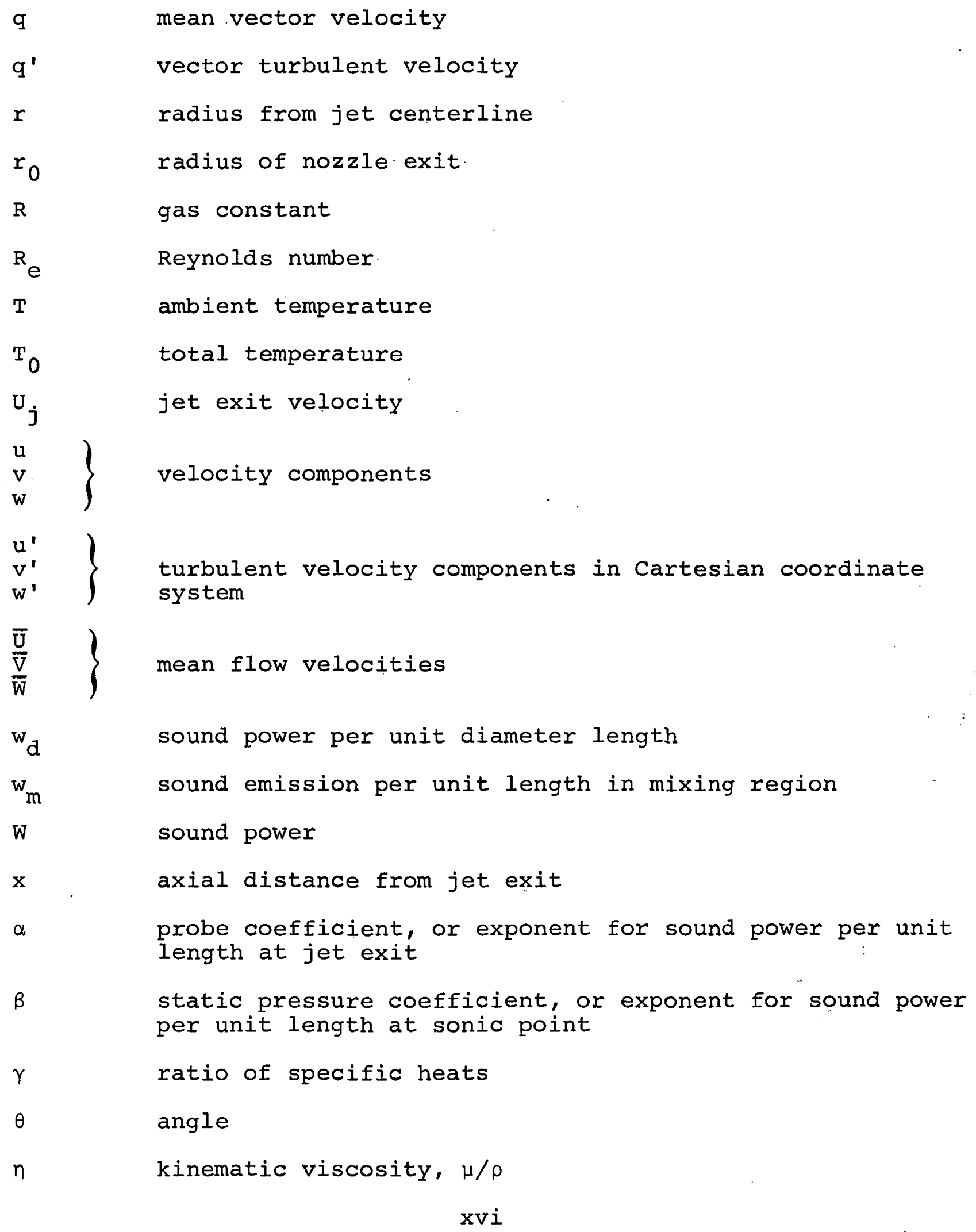




$\begin{array}{ll}\rho & \text { density } \\ \rho_{a} & \text { density of ambient air } \\ \rho_{j} & \text { density of jet } \\ \sigma & \text { pressure correlation scale } \\ \tau_{0} & \text { mean viscous shear stress }\end{array}$




\subsection{INTRODUCTION}

With the development of supersonic transports and military aircrafts, the supersonic exhaust velocities have made the problem of jet noise during take-offs quite critical without suppression devices at airports located in the metropolitan areas. Basic information regarding the noise generation mechanisms and suppression phenomena are required for the development of efficient suppressors for supersonic jets. Only limited experimental data was available for plain jets with supersonic exhaust Mach numbers, and also the available flow and acoustic information on the methods of reducing the noise from high velocity jets was limited. To obtain basic flow and acoustic knowledge for subsonic and supersonic jets a theoretical and experimental investigation was initiated several years ago and some of the results are presented in Refs. 1 - 10 for both plain supersonic jets and with suppressors. Various supersonic jet noise suppressor configurations were selected to obtain fundamental information regarding the necessary flow modifications to achieve large noise suppression.

Initial investigations were conducted to determine the flow and acoustic characteristics of subsonic and supersonic jets produced with a convergent nozzle. The flow velocity and the impact pressure fluctuations were determined over a jet Mach number range of 0.6 to 1.4 for a 2 in. diameter convergent nozzle in Ref. 4. Also, the near- and far-field sound pressure levels were determined for subsonic and supersonic Mach numbers, and from the far-field microphone measurements the sound power spectra were determined for various jet Mach numbers. It was observed from these results that the core region for subsonic jets extended over the initial five diameters from the nozzle exit, as observed also by other investigatorsil-15, and in the fully developed turbulent jet flow the velocity decreased inversely as the distance. For supersonic jet Mach numbers the sonic distance from the nozzle exit increased approximately as $\mathrm{M}_{j}^{2}$. Once the jet flow became subsonic the velocity decreased as $\mathrm{x}^{-1}$.

The sound pressure level for subsonic jet Mach numbers of 0.6 to 1.0 decreased monotonically from the jet axis for a constant radial distance from the nozzle exit as discussed in Ref. 4. But for supersonic jets from a convergent nozzle the sound pressure level was nearly constant over most of the angular positions. The power spectra of the subsonic jets were similar with the peak occurring at approximately $4 \mathrm{KHz}$, and for a Mach 1.4 jet the peak occurred at a frequency of $5 \mathrm{KHz}$. Near-field sound pressure level distributions were similar for subsonic jets, but for supersonic jets the sound pressure distribution was quite different because of acoustic radiation from the supersonic region. The acoustic radiation distribution from the supersonic jets increased from 
the nozzle exit to a peak value near the sonic location as observed also in Refs. 11 and 16. From the near-field measurements the overall acoustic power levels were determined and the values agreed with the far-field measurements for the supersonic jets.

The overall sound power levels determined from the far-field microphone measurements were compared with the subsonic theory of Lighthillil and supersonic theory of Nagamatsu and Horvay 3 in Refs. 4 - 6. For subsonic jets the overall acoustic power increased as $\mathrm{U}^{8}$ as predicted by Lighthill, but for a supersonic jet from a convergent nozzle the presence of the shock bottles increased the acoustic power as $\mathrm{U}^{12}$ over a Mach number range of 1.0 to 1.4 . Dosanjh and associates 18 also observed the additional acoustic radiation from the shock bottles at supersonic Mach numbers. The number of the shock bottles and the length of the supersonic region are functions of the jet Mach number as discussed in Refs. 4 , 5, and 19. With a properly designed convergent-divergent nozzle the flow at the nozzle exit is parallel to the axis with the static pressure equal to the ambient pressure so the shock bottles are eliminated as shown in Refs. 16, 20 and 21 .

The acoustic radiation from subsonic and supersonic jets is due to the turbulent velocity fluctuations as formulated by Lighthil117 and Ribner22. Besides the velocity fluctuations the turbulent shear stress in the jet will contribute to the static pressure fluctuations in fully developed subsonic turbulent flow as shown originally by Kraichnan23. For supersonic jets the supersonic mixing region produces Mach wave radiation as discussed in Refs. 24 - 26. To determine the distributions of mean and fluctuating velocities and the static pressure fluctuations for subsonic and supersonic jets, investigations were initiated to survey the jet plume with impact pressure and total temperature probes, and piezoelectric impact and static pressure probes, and the results of the surveys are presented in Refs. 4, 6 and 9.

For subsonic jet Mach numbers of $0.6,0.70,0.85$, and 1.0 , the contours of constant Mach numbers in the jet plume were quite similar for a convergent nozzle in Ref. 6 . On the axis of the nozzle the core length for these subsonic Mach numbers extended over the initial four diameters and downstream in the fully developed subsonic turbulent jet flow the axial velocity decayed as $\mathrm{x}^{-1}$, which was also observed in Refs. 4 and 20 for subsonic jets. But for supersonic jet Mach numbers of 1.2 and 1.4 the length of the supersonic region on the axis increased approximately as $M{ }_{j}$, and consequently, the contour for sonic Mach number extended over a larger portion of the jet plume at higher supersonic Mach numbers. It was also observed in these surveys of the jet plume that the subsonic mixing region surrounding the supersonic flow region increased rapidly with the distance from the nozzle exit. 
At these supersonic jet Mach numbers with shock bottles the axial velocity in the fully developed subsonic turbulent flow region decayed as $x^{-1}$ like the subsonic jets.

With the piezoelectric impact pressure probe the rms output of the probe is related to the product of the local mean density and velocity and the fluctuating velocity for subsonic flows. The piezoelectric impact pressure probe characteristic was determined by means of the hot-wire and the laser doppler velocimeter technique which measures the local mean and fluctuating velocities without disturbing the flow as discussed in Ref. 27. Detailed surveys of the jet plumes were conducted with the small piezoelectric impact probe over a jet Mach number range of 0.6 to 1.4 and the contours of constant rms piezoelectric probe output are presented in Ref. 6 . For subsonic Mach numbers of 0.6 to 1.0 the contours of the rms impact pressure fluctuations were quite similar with peak fluctuations occurring in a toroidal region located over an axial distance of approximately 3.5 to 6 diameters with the center of the region located approximately one nozzle radius from the axis. Other investigators in Refs. 28 - 31 have observed the turbulent velocity fluctuations with hot-wire in subsonic jets and have determined that the peak turbulent intensities occurred in the mixing region located at a radial location equal approximately to the nozzle radius. For supersonic jet Mach numbers of 1.2 and 1.4 with a convergent nozzle the peak piezoelectric impact pressure fluctuations for a given rms gage output occurred close to the sonic velocity contours. In the subsonic mixing region the contours of constant pressure fluctuations were similar to those observed for sonic and subsonic jets as shown in Ref. 6 .

The static pressure fluctuations due to the turbulent velocity fluctuations and shear in the jet plume have been investigated by various authors $9,32-34$ for determining the acoustic sources for subsonic and supersonic jets. By the use of the laser doppler velocimeter technique for determining the mean and fluctuating turbulent velocities, the piezoelectric static pressure characteristics were determined over a Mach number range of 0.3 to 1.0 in Ref. 27. On the axis of the fully developed subsonic turbulent jet the rms static pressure fluctuation due to turbulence is proportional to the product of the local mean density and the square of the rms turbulent velocity fluctuation according to Kraichnan's theory ${ }^{2}$. This relationship was used to determine the static pressure probe constant in Ref. 27. With this slender piezoelectric static pressure probe the rms static pressure fluctuation distributions in subsonic and supersonic jets were determined in Ref. 9 for a convergent nozzle. The contours of constant rms static pressure fluctuation were similar for jet Mach numbers of $0.6,0.7,0.85$, and 1.0 . With the peak static pressure fluctuations occurring at the jet mixing region with the ambient air, at 
a radial location slightly larger than the nozzle radius. Also, the piezoelectric static pressure probe output decreased continuously across the jet boundary for these subsonic Mach numbers indicating the presence of the acoustic waves outside the jet plume. The contours of the rms static pressures were entirely different than the contours for the piezoelectric impact pressure. probe output for these subsonic jet Mach numbers. Similar static pressure fluctuations for subsonic jets were observed in Ref. 31 at slightly lower Mach numbers.

The rms static pressure fluctuation contours for supersonic Mach numbers of 1.2 and 1.4 for a convergent nozzle were entirely different than those observed for subsonic Mach numbers of 0.6 to 1.0. Because of the presence of the shock bottles at these Mach numbers the peak rms static pressure fluctuations occurred in the supersonic mixing region, within the sonic Mach number contour as shown in Ref. 9. Also the variations of the static pressure fluctuations outside the supersonic jet plumes were very different than those existing for subsonic jets. For supersonic jet Mach numbers there is the presence of Mach waves produced by the turbulent eddies at the edge of the jet moving at supersonic Mach number relative to the ambient velocity of sound as shown by spark shadowgraph photographs in Refs. 6, 16, and 18. These Mach waves are not present for air jets operated at room temperature with subsonic exhaust Mach numbers as shown clearly in Ref. 6 with the shadowgraphs obtained with a short duration spark source. In the fully developed subsonic turbulent jet the contours of the static pressure fluctuations for jet Mach numbers of 1.2 and 1.4 were similar to those observed with sonic and subsonic exhaust velocities. Again the contours of the rms static pressure fluctuations were very different than the contours of the rms piezoelectric impact pressure fluctuations for these supersonic Mach numbers.

The suppression of the jet noise.from supersonic jets is very complicated and difficult:because a large reduction in the noise level is required with minimum thrust loss to meet the noise regulations. Various suppressor configurations have been investigated and the results are presented in Refs. $1,2,7-10,35,-37$. $A$ long shroud suppressor with small rods was investigated in Refs. 1 , 2 and 9 to determine the suppression of a Mach 1.4 jet and the corresponding decrease in the jet velocity by inducing an appreciable amount of ambient air. With the six small rods located near the nozzle exit and induced mass flow the overall power level of the Mach 1.4 jet was decreased by $14.2 \mathrm{db}$ and the Mach number at the shroud exit was approximately 0.8 with a nearly uniform velocity distribution across the exit.

To achieve large noise level reductions with a much shorter length than the single shroud, multiple tubes and multiple shrouds were 
investigated in Refs. 7 and 8. Multiple tubes were investigated by other authors in Refs. $35-37$. It was shown in the supersonic jet noise theory of Nagamatsu and Horvay 3,5 that the sonic location on the jet axis for supersonic jets for both cold and hot jets over a range of nozzle diameters was proportional to the square of the jet Mach number and the diameter of the nozzle. Thus, for a given pressure ratio across the nozzle for supersonic Mach numbers the length of the supersonic region is dependent upon the nozzle or tube diameter, and by the use of multiple tubes instead of a single nozzle the supersonic length was drastically decreased. The sonic location for a Mach number of 1.4 with the 1-9/16 inch diameter convergent nozzle was approximately 20 in., and for the corresponding 191 tubes of 0.115 inch diameter the sonic location was approximately $l$ in. as shown in Ref. 7 . Hence, the supersonic region with large acoustic radiation was drastically decreased with an overall sound power. level reduction of $15 \mathrm{db}$ for a Mach 1.4 jet and a thrust loss of 10.2 percent. By adding 191 shrouds to the 191 tubes the ambient air was induced into these shrouds with a corresponding decrease in the jet velocity and the shroud also shielded the large acoustic radiation from the supersonic region of the jet. With this method the overall sound power level of the Mach 1.4 jet was reduced by approximately $20 \mathrm{db}$ with a thrust loss of nearly 27 percent. This large thrust loss was caused primarily by the 191 shrouds of 6 inch length which had a large wetted surface which contributed approximately 12 percent to the thrust loss.

To investigate the supersonic jet noise suppression with a smaller number of tubes for practical considerations, 50 tube and 50 shroud suppressor configurations were studied in Ref. 8 with various tube lengths. Both flow and acoustic characteristics and thrust losses were determined for a supersonic jet Mach number of 1.4. The overall sound power level reductions with the 50 tubes of $1 / 4$ to 4 inch lengths were approximately $12 \mathrm{db}$ because the merged Mach numbers were about sonic for these tube lengths. With 50 holes the overall sound power level reduction from a Mach 1.4 jet was $15 \mathrm{db}$ with a thrust loss of nearly 17 percent because of the low base pressure. The thrust loss was decreased. to 6.8 percent for a tube length of. $1 / 4$ inch and for tube lengths of 1,2 , and 4 inches the thrust loss was 3.3 percent. The base pressure increased with the tube length because a greater area was available for ambient air to enter the tube bundle. By adding the 50 hexagonal shrouds of 6 inch length to the tubes of 1,2 , and 4 inches, the overall sound power level reduction of a Mach 1.4 jet was increased. For a tube length of 4 in. with multiple shrouds the overall sound power level was decreased by $20.3 \mathrm{db}$ with a thrust loss of nearly 27 percent. The thrust loss with the shrouds was large because of the large wetted area in the hexagonal shrouds as discussed in Refs. 8 and 10 . 
This report presents the results on the flow and acoustic characteristics of subsonic and supersonic jets from a convergent nozzle, and the thrust characteristics for various supersonic suppressor configurations. A brief description of the experimental facilities used to obtain flow and acoustic data as well as the facilities used to calibrate the instrumentation will be presented. Mean and fluctuating flow parameters in the subsonic and supersonic jets were determined with impact pressure and total temperature probes for mean quantities and the fluctuating velocities and pressures with the laser doppler velocimeter and piezoelectric impact and static pressure probes. The laser doppler velocimeter technique was used to calibrate the piezoelectric impact and static pressure probes. The summary of the results on the subsonic and supersonic jet plume surveys with these instrumentation will be presented as well as the corresponding nearand far-field acoustic results. A summary of the flow, acoustic, and thrust characteristics for single and multiple shrouds and multiple tubes will be presented for supersonic jet Mach number of 1.4. The acoustic results for the multiple tube configurations are analyzed by using the subsonic jet noise theory of Lighthill17 and supersonic jet noise theory of Nagamatsu and Horvay 3,5 . 


\subsection{EXPERIMENTAL FACILITIES AND PROCEDURE}

\subsection{Test Facilities}

Two compressor systems are available to supply the air to the nominal 2 and 6 in. diameter nozzles, Fig. 1 , for investigating flow and acoustic characteristics of subsonic and supersonic jets. One system consists of two $800 \mathrm{hp}$ reciprocating compressors which can supply $5 \mathrm{lbs} / \mathrm{sec}$. Of air at 500 psia and a gas fired heater is available to heat the air to $900^{\circ} \mathrm{F}$. These reciprocating compressors are used to supply air to the 2 in. diameter. nozzle at supersonic jet Mach numbers. The second system consists of two banks of Fuller compressors with each bank powered with a 350 hp motor. With these compressors it is possible to supply $11 \mathrm{lbs} / \mathrm{sec}$. of air at 30 psia for operating the 6 in. nozzle over a Mach number range of 0.10 to 0.9 .

Convergent nozzles with exit diameters of 2 and $1-9 / 16$ in. were used with the outdoor flow and acoustic facility shown in Fig. 1 . This facility is equipped with a trolley system so that axial flow surveys in the jet plume can be conducted as well as near-field axial microphone surveys, and the flow and acoustic results for subsonic and supersonic jets with and without suppressors are presented in Refs. I to 10.

Since it is difficult, at times, to conduct detailed surveys of the subsonic and supersonic jet plumes in the outdoor flow and acoustic facilities because of the effects of wind velocity on the jet plume, a 1 in. diameter convergent nozzle was utilized in the Fuller compressor room as shown in Fig. 2. The air to this nozzle was supplied by a four-stage reciprocating compressor powered by a $200 \mathrm{hp}$ motor. With this nozzle the distributions of the mean velocity, and piezoelectric impact and static pressure fluctuations were determined over a Mach number of 0.3 to 1.4 and the results are presented in Refs. 6 and 9. Also, it was possible to obtain shadowgraph and schlieren photographs of the subsonic and supersonic jets with a 0.4 microsecond spark source. With a convergent nozzle at a Mach number of 1.4 there are eight shock bottles in the supersonic region ${ }^{6}$.

For calibrating the piezoelectric impact and static pressure probes, and the sputtered platinum wedge probe, a laser doppler velocimeter method has been developed as discussed in Refs. 27 and 38 . Small $1 / 2$ and $3 / 4$ in. nozzles were used to develop the laser method for determining the mean and fluctuating velocities for subsonic and supersonic jets, and the laser and the nozzle are shown in Fig. 3. The very clean dry house air supply was used to produce jet Mach numbers of 0.3 to 1.4 , and a seeding method for putting small particles into the air stream was developed for the nozzle. 
At a Mach number of 0.3 a hot-wire was used to determine the turbulent velocity fluctuations, which agreed with the laser doppler velocimeter measurements.

\subsection{Procedure}

With the 2 in. diameter nozzle located outdoors, Fig. 1, two separate runs were made to obtain the flow and acoustic data. The reservoir pressure was selected to produce jet Mach numbers of 0.6 to 1.4 and the reservoir pressure was maintained constant during the run with a Fisher flow regulator. Total pressure and total temperature surveys along the axis of the jet were made from the nozzle exit to 40 nozzle diameters downstream, and these tests were made only when the ambient wind velocity was less than $10 \mathrm{mph}$.

For the 2 in. convergent nozzle the far-field acoustic measurements were made with the microphone placed at eight angular positions in a horizontal plane at a fixed $10 \mathrm{ft}$. radius from the jet exit as shown in Fig. 1. Before each test the microphone was calibrated with the $B \& K$ piston phone calibrator, and the microphone output at each angular location was recorded on a General Radio tape recorder. Also, the rms voltmeter and $B \& K$ sound pressure level meter readings were obtained at each angular position. Axial near-field surveys were made with two microphones located at various radial distances from the nozzle exit, and the microphone outputs were connected to the Ballantine rms voltmeter to obtain the sound pressure level at each location.

Surveys with piezoelectric impact and static pressure probes were conducted along the axis for the 2 in. convergent nozzle with the trolley system shown in Fig. 1 and radial and axial surveys with these probes were made for the 1 in. nozzle with the probe equipment shown in Fig. 2 over a Mach number range of 0.3 to 1.4 . Axial surveys with these probes were made for the $1 / 2$ and $3 / 4$ in. diameter nozzles with the laser doppler velocimeter facility shown in Fig. 3 over subsonic to supersonic Mach numbers. All of these tests with piezoelectric probes were conducted with room temperature jets. 


\subsection{INSTRUMENTATION AND CALIBRATION}

3.1 Instrumentation

One of the objectives of the fundamental investigation of the supersonic jet noise phenomenon was to determine the noise sources in the jet plume. Thus, instrumentation were developed to measure the mean and fluctuating flow parameters in the subsonic and supersonic jets. These results would indicate the location of maximum velocity and static pressure fluctuations in the jet plume. The probes to measure mean and fluctuating flow parameters are presented in Fig. 4.

The mean velocity and total temperature were determined in the jet plume from the measurements of the local impact pressure and total temperature. The opening of the total pressure probe, $F$, was $0.055 \mathrm{in.}$, and the opening of the total temperature probes were 0.055 and 0.125 in., probes $J$ and B in Fig. 4. For both of these probes the smaller diameter tips were used to survey the $1,1 / 2$ and $3 / 4$ in. nozzles. $A 1 / 8$ in. diameter impact and static pressure probe, $K$, with a hemispherical nose was used for surveys of the subsonic flow regions.

A $1 / 4$ in. diameter piezoelectric quartz Kistler gage was. used as the pressure transducer for both impact and static pressure probes. The response time of this gage was approximately $20 \mathrm{\mu sec}$. The face of the gage was exposed as a piezoelectric impact pressure probe, $\mathrm{E}$, as shown in Fig. 4, which was used for the axial survey for the 2 in. diameter nozzle. To reduce the opening diameter to $0.055 \mathrm{in}$. anextension was placed ahead of the piezoelectric gage with 0.055 in. diameter opening to the cavity in front of the pressure gage, probe $\mathrm{H}$ of $\mathrm{Fig}$. 4. This smaller diameter impact pressure, probe was used to survey the jet plume for $1,1 / 2$ and $3 / 4$ in. diameter nozzles.

Fluctuating static pressures in the jet plume were determined with sharp and blunt tipped static pressure probes with a $1 / 4$ in. diameter piezoelectric quartz Kistler pressure gage and a $1 / 4$ in. diameter B\&K microphone, $A$, as shown in Fig. 4. Only the sharp tipped probes were used in the supersonic region of the flow and both sharp and blunt probes were used in the subsonic flow regions. Two static pressure probes of $5 / 16$ in. diameter were constructed with sharp, $C$, and blunt, D, tips, and the static pressure was transmitted through small orifices connected to the small cavity in front of the pressure gage. A $1 / 8$ in. diameter static pressure probe, G, was constructed with a sharp tip as shown in Fig. 4. Small static pressure orifices were located around the probe and this was connected to a constant diameter opening to the face of the $1 / 4$ in. diameter quartz gage. The gage characteristics for the 
static pressure probe were determined by the use of the laser doppler velocimeter method which gave the local mean velocity and the turbulent velocity fluctuations.

$B \& K$ condenser microphones of $1 / 4$ and $1 / 2$ in. diameters were used to obtain near- and far-field acoustic pressure data with a cathode follower for a frequency response of $20 \mathrm{~Hz}$ to $40 \mathrm{KHz}$. The microphone was calibrated with a $B \& K$ pistonphone calibrator which produced an oscillating dynamic pressure of $124 \mathrm{db}$ re. $0.0002 \mu \mathrm{bar}$ at $250 \mathrm{~Hz}$ before each test. The output of the microphone was connected to a Ballantine true rms voltmeter, $B \& K$ sound level meter, and General Radio tape recorder which had a frequency response of $15 \mathrm{~Hz}$ to $20 \mathrm{KHz}$. The tape recordings were analyzed using a $\mathrm{B} \& \mathrm{~K}$ $1 / 3$ octave band analyzer coupled to a Hall squaring circuit and a digital integrating voltmeter, and a detailed discussion of the data analysis is presented in Ref. 1 .

\subsection{Calibration Shock Tube}

An 8 in. diameter shock tube, Fig. 5, with a driver length of 5-ft. and driven length of $36 \mathrm{ft}$. was constructed for calibrating the piezoelectric pressure and sputtered platinum heat gages used to obtain pressure and heat transfer data in the hypersonic shock tunnel at high Mach numbers, and a detailed description of this equipment is presented in Ref. 39. By placing the gages to be calibrated near the end of the driven tube and using a weak shock wave the pressure increase across the shock wave and the heat transfer from the flow can be calculated by the use of the shock wave relationship. The pressure and temperature ahead of the shock wave and the shock velocity are determined for each test. Piezoelectric impact and static pressure probes and the sputtered platinum heat gage probes are mounted at the end of the shock tube on the center Iine. Pressure increases across both the incident and reflected shock waves were used for the calibration of the pressure gages. The heat transfer rates after the incident shock waves were calculated from the results presented in Ref. 40.

\subsection{Laser Doppler Velocimeter}

It was observed in Ref. 4 for a convergent nozzle at a Mach number of 1.4 that the peak piezoelectric impact pressure occurred in the vicinity of the sonic velocity on the axis. Thus, an investigation was initiated to measure the local mean and fluctuating velocities in subsonic and supersonic jets with the laser doppler velocimeter without disturbing the flow. These local velocities were necessary to calibrate the piezoelectric impact and static pressure probes and the sputtered platinum wedge heat gage probe 27 . The initial laser doppler velocimeter shown in Figs. 3 and 6 was used to calibrate the laser results for the turbulence level with a hot wire at a Mach number of 0.3 as discussed in Ref. 
38 with a low power laser.

For higher jet velocities it was necessary to use a two watt continuous wave (CW) laser of wave length of $5145 \mathrm{~A}$. The 0.5 and 0.75 in. nozzle was mounted on the lathe carriage bed so that mean and fluctuating velocities at various locations in the jet plume could be measured as indicated in Fig. 6. It was necessary to inject 0.3 micron size alumina particles from a fluidized particle bed to determine the mean and fluctuating velocities in supersonic flows. A detailed description of the IDV technique and some of the mean velocity and turbulence level results for subsonic and supersonic jets are presented in Refs. 27 and 41 .

\subsection{Piezoelectric Impact and Static Pressure Probes:}

A Kistler quartz pressure transducer of $1 / 4$ in. diameter was used for measuring the impact pressure fluctuations with probe tip openings of $0.055 \mathrm{in.}$ and $0.25 \mathrm{in}$. diameter as shown in Fig. 4 . The piezoelectric pressure gage output as a function of the magnitude of the pressure jump across the shock wave was determined by the use of the calibration shock tube. By varying the pressure in the driven tube and the driver. of the shock tube, various pressure jumps across the incident and reflected shock waves were produced, and the gage output as a function of the pressure increase across the shock wave is presented in Fig. 7. After the incident shock wave there is an induced flow velocity while back of the reflected shock wave the flow is brought to rest. Within the experimental accuracy the pressure gage output was not affected by the flow velocity.

The static probes of $1 / 8$ and $5 / 16$ in. diameters with blunt and sharp tips shown in Fig. 4 also used a $1 / 4$ in. diameter quartz pressure transducer. This probe was also calibrated in the shock tube to determine the output as a function of the pressure jump across the shock as well as the response time. A static pressure probe with a $1 / 4$ in. diameter $B \& K$ microphone and a hemispherical nose was also used to measure the static pressure fluctuations in the subsonic flow regions. The output. of the microphone as a function of the pressure increase across weak shock waves was also determined in the calibration shock tube. 
4.0 ANALYSIS OF PIEZOELECTRIC IMPACT AND STATIC PRESSURE FLUCTUATIONS AND TURBULENT VELOCITY FLUCTUATIONS

\subsection{Analysis of Piezoelectric Impact Pressure Fluctuations}

For investigating the subsonic and supersonic jet noise generation phenomena, it is necessary to determine the mean and fluctuating velocities in the plume. Since the hot-wire is too fragile to determine the mean and fluctuating velocities at high velocities, the piezoelectric impact pressure probes $4,27,33,34$ and laser doppler velocimeter technique $27,38,41$ have been developed for obtaining the turbulent velocity fluctuations in high subsonic and supersonic jet exhaust velocities.

For a fully developed. uniform turbulent flow at low subsonic velocities, the turbulence can be considered roughly to be isotropic so that the turbulent velocity components can be expressed as

$$
\overline{\mathrm{u}^{\prime 2}}=\overline{\mathrm{v}^{\prime 2}}=\overline{\mathrm{w}^{\prime 2}}=\frac{1}{3} \overline{\mathrm{q}^{\prime 2}}
$$

In the Cartesian coordinate system with a uniform mean flow in the $\mathrm{x}$-direction the velocity components can be expressed as

$$
\begin{aligned}
& \mathrm{u}=\overline{\mathrm{U}}+\mathrm{u}^{\prime} \\
& \mathrm{v}=\mathrm{v}^{\prime} \\
& \mathrm{w}=\mathrm{w}^{\prime}
\end{aligned}
$$

since $\bar{V}$ and $\bar{W}$ vanish in the assumed uniform turbulent flow. In a uniform low speed homogeneous isotropic turbulent flow there will be mean static pressure, $\bar{p}$, and the static pressure is given by

$$
p=\bar{p}+\left\langle p^{\prime}\right\rangle+\tilde{p}
$$

where $\left\langle p^{\prime}\right\rangle$ is the static pressure fluctuation caused by turbulence given by Kraichnan 23 and $\tilde{\mathrm{p}}$ is the acoustic pressure fluctuation present in the flow. The pressure fluctuations caused by the turbulence will be assumed to propagate close to the velocity of the flow, while the pressure fluctuations due to the acoustic field will propagate at the velocity of: sound in low speed flows.

At the stagnation region of the piezoelectric impact pressure probe, the turbulent flow is brought to rest through large 
gradients of flow properties. In the stagnation region the spatial changes will exceed the temporal ones. Under these conditions the total pressure along a streamline in a low speed turbulent flow from Bernoulli's equation is given by

$$
\mathrm{p}_{\mathrm{T}}=\mathrm{p}+\frac{1}{2} \rho \mathrm{q}^{2}
$$

where $q$ is the vector velocity consisting of a mean vector velocity and a vector turbulent velocity. By substituting Eqs. (2) and (3) into. Eq. (4) and neglecting higher order terms of the turbulent velocity fluctuations, Eq. (4) can be split into mean and fluctuating components

$$
\begin{aligned}
& \overline{\mathrm{p}}_{\mathrm{T}}=\overline{\mathrm{p}}+\frac{1}{2} \bar{\rho} \overline{\mathrm{U}}^{2} \\
& \mathrm{p}_{\mathrm{T}}^{\prime}=\left\langle\mathrm{p}^{\prime}\right\rangle+\tilde{\mathrm{p}}+\bar{\rho} \overline{\mathrm{u}} \mathrm{u}^{\prime}
\end{aligned}
$$

The contribution to the impact pressure fluctuation by the static pressure fluctuations due to turbulence $\left\langle p^{\prime}\right\rangle$ for low speed turbulent flow should be small.

Nakamura 33 and Fuchs ${ }^{34}$ have obtained experimental data in low speed turbulent flow with microphone impact and static pressure probes and confirmed the low level of static pressure fluctuations due to turbulence. Thus, the piezoelectric impact pressure fluctuation with a total head probe configuration in a low speed turbulent flow with negligible acoustic field is given by

$$
\mathrm{P}_{\mathrm{T}}^{\prime}=\alpha \bar{\rho} \overline{\mathrm{U}} \mathrm{u}^{\prime}
$$

which is dependent on the probe coefficient $\alpha$, local axial velocity fluctuations, mean velocity, and mean density. The piezoelectric impact pressure probe in principal can be used in place of the hot wire to investigate the fluctuations in unsteady flows as discussed by Nagamatsu9,27, Siddon 32 , and Fuchs ${ }^{34}$. The available references on the actual correlation of the piezoelectric impact pressure probe and hot wire is still very limited ${ }^{9}$.

An investigation was undertaken to obtain information regarding the piezoelectric impact pressure fluctuations and the local turbulence level by the use of hot wire and laser doppler velocimeter 27 . The mean and fluctuating velocities were determined along the axis 
for the 3/4 in. diameter nozzle, Figs. 3 and 6 , with the Disa constant temperature hot-wire for a jet Mach number of 0.3 and the results are presented in Fig. $8 \mathrm{a}$. At this jet Mach number the mean and fluctuating velocities were determined with the hot-wire and laser doppler velocimeter method. The core region extended over the initial four diameters with the turbulence level on the axis increasing from a value of 1.9 percent at a location one-half diameter from the nozzle exit, to a maximum of 14 percent at a distance of 8 diameters before decreasing to 9.4 percent. at 16 diameters.

With the small piezoelectric impact pressure probe with an opening at the tip of 0.055 in., Fig. 4, the rms output of the probe was determined along the axis. Using the gage characteristics presented in Fig. 7, the rms output value was converted to rms impact pressure fluctuation in psi. This impact pressure fluctuation, the mean velocity from the hot-wire, and the mean density in the jet were used in Eq. (7) to determine the turbulent velocity fluctuation, and the results are presented in Fig. 8 a. Over the initial 8 diameters the turbulence levels determined by the piezoelectric impact pressure probe and the hot-wire are practically identical indicating that the turbulence level given by Eq. (7) is valid for subsonic flows. Downstream of the peak turbulence at 8 diameters the turbulence levels determined by the piezoelectric impact pressure probe were slightly less than the values obtained with the hot-wire, and this may be due to the low probe output, at low velocities.

Since the hot-wire in higher velocity flows does not withstand the higher dynamic pressure without failures, the laser doppler technique was used to determine the mean and turbulent velocities for jet Mach numbers of $0.6,1.0$ and 1.2 , and the results for a Mach 1.2 jet are presented in Fig. 8b. Also, in this figure the mean velocities determined along the axis from the pitot and total temperature probes are presented, and the mean velocities determined with the LDV technique agreed reasonably well with the values determined from the probe measurements. The small piezoelectric impact pressure probe was used to determine the impact pressure fluctuations along the axis of the jet.' The rms pressure fluctuations was used to determine the turbulent velocity fluctuations by the use of Eq. (7) and the results are presented in Fig. $8 \mathrm{~b}$. Over the initial 4 diameters from the nozzle exit the turbulence levels determined with the piezoelectric impact probe were lower than the results from the LDV, but at axial locations of 14,16 , and 18 diameters the turbulence levels determine from the LDV measurements agreed within the experimental accuracy with the turbulence levels obtained from the piezoelectric pressure probe data. 


\subsection{Analysis of Piezoelectric Static Pressure Fluctuations}

Since the acoustic radiation from the subsonic and supersonic jets is dependent upon the turbulence within the jet plume, the knowledge regarding the static pressure fluctuations due to turbulence and shear is useful for understanding the noise generation mechanism. An investigation was conducted to study the characteristics of static pressure probes with a piezoelectric element and a microphone as the fluctuating static pressure transducers. In the past several years investigators have used microphones and piezoelectric pressure gages to measure the fluctuating pressures for impact and static pressure probe configurations in subsonic $32-34$ and supersonic $2-9$ jet flows.

For a uniform low speed homogeneous isotropic turbulent flow with a mean velocity $\bar{U}$ and turbulent velocity components $u$ ', $v !$, and $w^{\prime}$, Kraichnan 23 derived the expression for the mean-square static pressure fluctuations associated with the isotropic subsonic turbulent flow as.

$$
\left\langle\mathrm{p}^{\prime 2}\right\rangle=\overline{\mathrm{p}}^{2} \mathrm{u}^{\prime 4} \mathrm{c}^{-4}
$$

where $c$ is the velocity of sound. And the ratio of the rms turbulent velocity in the flow direction to the local velocity of sound can be expressed as the local turbulence Mach number

$$
M^{\prime}=u^{\prime} / c \text {. }
$$

In subsonic turbulent flow with shear gradient the mean-square pressure fluctuation is given by

$$
\left\langle\mathrm{p}^{\prime 2}\right\rangle=\frac{4}{15} \tau_{0}^{2} \mathrm{u}^{\prime 2} \cdot \eta^{-2} \sigma^{-\frac{2}{2}}
$$

where $\tau_{0}$ is the mean viscous shear stress, $\eta=\mu \% \rho$ is the kinematic, viscosity, and $\sigma$ is related to the area scale of the pressure correlation, defined in Ref. 23.

Besides the turbulent static pressure fluctuations there will be a pressure fluctuation in low speed flows due to a sound field being superimposed from outside the jet flow or generated aerodynamically in the flow, denoted by $\tilde{p}$. Thus, in a low speed turbulent jet flow there will be a static pressure in the flow field given by 


$$
p=\bar{p}+\left\langle p^{\prime}\right\rangle+\tilde{p}
$$

and $\left\langle p^{\prime}\right\rangle$ is the static pressure caused by turbulence, Eq. (8) or Eq. (10), depending upon the mean shear in turbulent flow. The pressure fluctuations caused by turbulence will be assumed to propagate close to the velocity of the air flow, while the pressure fluctuations due to the acoustic field will propagate with the velocity of sound for low speed flow. Along the jet axis the static pressure determined with piezoelectric and microphone static pressure probes, Fig. 4, will be given by Eq. (8), and can be expressed as

$$
\left\langle\mathrm{p}^{\prime}\right\rangle=\beta \bar{\rho} \mathrm{u}^{\prime 2}
$$

where $\beta$ is the static pressure coefficient.

The rms magnitude of the axial static pressure fluctuations determined with the $1 / 8$ in. diameter probe with sharp tip, Fig. 4, are presented in Fig. 9a as a function of the distance from the $3 / 4$ in. diameter nozzle exit, Fig. 3, for a jet Mach number of 0.3 . Also, the core, mixing, and turbulent regions determined from the axial mean velocity distribution are labeled in this figure. The peak rms static pressure fluctuation occurred at the end of the core region and the pressure decreased continuously in the mixing and turbulent regions. Over the fully developed turbulent region the static pressure fluctuations along the jet axis decreased rather slowly.

Using the hot-wire measurements for the mean and fluctuating velocities and the density determined from the pitot pressure and total temperature data, the rms magnitude of the static pressure fluctuations were calculated by the use of $\mathrm{Eq}$. (12) and the results are presented in Fig. 9a. Over the initial 8 diameters the piezoelectric static pressure: probe results were higher than the pressures calculated from the hot-wire data. In the fully developed turbulent region on the axis Eq. (12) is valid, and in this region the measured rms static pressure fluctuation and the value calculated from the hot-wire results agreed within experimental accuracy. Similar calculations for the static pressure fluctuations were made with the fluctuating velocities determined with the laser doppler velocimeter method,. Figs. 3 and 6 , and the results are presented in Fig. 9a. The static pressure fluctuations were slightly higher than the values determined from the hot-wire data over the initial region of the jet plume. And at an axial location of 8 diameters the rms static pressure fluctuations determined from the hot-wire and the IDV measurements agreed with the magnitude of the static 
pressure fluctuation measured with the static pressure probe. This agreement indicates that the acoustic pressure waves at low Mach numbers are small compared with the static pressure fluctuations associated with the fully developed turbulent flow along the axis.

In Fig. 9b the axial rms static pressure fluctuations determined with the piezoelectric static pressure probe and calculated from the LDV turbulence level measurements are presented as a function of the distance from the $3 / 4$ in. nozzle at a Mach number of 0.6 . The static pressure fluctuations determined with the probe increased from the nozzle exit to a peak value located at the end of the core region. In the fully turbulent flow the magnitude of the static pressure fluctuations decreased. continuously with distance. The magnitude of static pressure fluctuations calculated from the LDV fluctuating velocities in Eq. (12) increased from the nozzle exit, similar to the increase observed with the probe. And at the end of the mixing region located at 8 diameters from the nozzle exit, the calculated static pressure fluctuation from the LDV measurement agreed with the value determined with the static pressure probe. Thus, the acoustic pressures present in the jet plume, were evidently small compared with the static pressure fluctuations associated with turbulence in a Mach 0.6 jet.

Similar comparison of the static pressure fluctuations determined with the piezoelectric static pressure probe and the LDV method was obtained for the $1 / 2$ in. diameter convergent nozzle at a Mach number of 1.2 and the results are presented in Fig. 27. At this Mach number the laser doppler velocimeter method became marginal because of the limitation of the electronic circuitry. An improved electronic circuit is being developed so as to determine the mean and fluctuating velocities at high supersonic Mach numbers. 


\subsection{SUBSONIC AND SUPERSONIC JET FLOW CHARACTERISTICS}

\subsection{Axial Distributions of Mean Velocity}

The axial variations of the flow velocity along the jet axis were determined for the 2 and 1 . in. diameter convergent nozzles, Figs. 1 and 2 , by means of the impact pressure and total temperature probes shown in Fig. 4. With the convergent nozzles the jet expands from the exit for pressure ratios greater than the critical value. For reservoir pressures higher than the critical value the flow expands to supersonic velocities with the presence of shock bottles for convergent nozzles as shown in Refs. 4, 6, 18 and 19. Under these conditions the flow Mach number near the nozzle exit was determined from the ratio of the impact pressure to the reservoir pressure, and farther downstream from the nozzle exit the local Mach number was determined from the ratio of the ambient pressure to the impact pressure. For subsonic flow regions the local Mach number; was determined from the ratio of ambient to impact pressure. These Mach numbers are given by the following equations:

$$
\begin{aligned}
& \frac{p_{o}^{\prime}}{p_{0}}=\left(\frac{(\gamma+1) M^{2}}{(\gamma-1) M^{2}+2}\right)^{\frac{\gamma}{\gamma-1}}\left(\frac{\gamma+1}{2 \gamma M^{2}-(\gamma-1)}\right)^{\frac{1}{\gamma-1}} \\
& \frac{p_{a}}{p_{0}^{\prime}}=\left(\frac{2 \gamma}{\gamma+1} M^{2}-\frac{\gamma-1}{\gamma+1}\right)^{\frac{1}{\gamma-1}}\left(\frac{\gamma+1}{2} M^{2}\right)^{-\frac{1}{\gamma-1}},
\end{aligned}
$$

and

$$
\frac{p_{a}}{p_{o}}=\left(1+\frac{\gamma-1}{2} m^{2}\right)^{\frac{\gamma}{2(\gamma-1)}}
$$

The local flow velocities in the jet were determined from the impact pressure and total temperature measurements. The relationship between the total temperature of the flow and the ambient temperature is given by

$$
\frac{T}{T_{0}}=\left(1+\frac{y-1}{2} M^{2}\right)^{-1}
$$

And thus knowing the local Mach number from Eqs. (13), (14), or (15) and the total temperature, the local temperature was calculated 
from this equation. By assuming a perfect gas, the local velocity of sound was calculated from

$$
\mathrm{c}^{2}=\gamma \mathrm{RT}
$$

and the local flow velocity by

$$
\mathrm{v}=\mathrm{MC}
$$

The axial flow velocities determined by this method for various jet Mach numbers for the 2 in. convergent nozzle 4 are presented in Fig. 10a. For subsonic jet Mach numbers of 0.6 to 1.0 the core region extended to approximately 5 diameters on the axis. For these subsonic Mach numbers the flow became fully turbulent at approximately 10 diameters from the nozzle exit. Downstream of this location in the fully developed turbulent flow region the velocity decreased as $x^{-1}$. At supersonic Mach numbers of 1.2 and 1.4 the sonic location on the axis moved downstream with the Mach number and for a Mach number of 1.4 the sonic location was at 14 diameters. In the fully developed subsonic turbulent flow region for these supersonic Mach numbers the velocity decreased as $x^{-1}$ similar to that observed for subsonic jets. With the 1 in. diameter convergent nozzle, Fig. 2, the core region as shown in Fig. $10 \mathrm{~b}$ extended over the initial 4 diameters fior the subsonic Mach numbers of 0.6 to 1.0 , and in the fully developed turbulent flow region the velocity decreased as $\mathrm{x}^{-1}$. The sonic location moved downstream at supersonic Mach numbers of 1.2 and 1.4. Again for these Mach numbers in the fully developed subsonic turbulent flow region the velocity decreased as $\mathrm{x}^{-1}$ similar to the results obtained for the 2 in. convergent nozzle. A more detailed discussion of the axial variation of mean velocity for the 2 and 1 in. convergent nozzles at subsonic and supersonic velocities are presented in Refs. 4 and 6.

\subsection{Axial Piezoelectric Impact Pressure Fluctuations}

The axial rms piezoelectric impact pressure fluctuations were determined for the convergent nozzles with 2 in. and 1 in. diameter in Refs. 4, 6, and 9 with the quartz piezoelectric impact pressure probes shown in Fig. 4. For the 2 in. nozzle the $1 / 4$ in. diameter probe was used to measure the impact pressure fluctuations over an axial distance of 40 diameters from the jet exit, and the results are presented in Fig. lla. It was shown by Eq. (7) that the rms output of the piezoelectric impact pressure probe was a function of the mean density and velocity and the turbulence 
velocity for a subsonic jet flow. Over the subsonic Mach number range of 0.6 to 1.0 , the variations of the impact pressure fluctuations with distance were quite similar with the peak fluctuations occurring at approximately 9 diameters from the jet exit. For subsonic Mach numbers in the, core region of approximately 5 diameters the impact pressure fluctuations are quite small compared to the peak value. The peak impact pressure fluctuations occur in the region where the primary jet is completely mixed with the ambient gas. After this region the jet decays as a fully established turbulent jet flow and the rms impact pressure fluctuation decays as $x^{-1.74}$ while the velocity decayed as $x^{-1}$ in this region as shown in Figs. 10a and $10 \mathrm{~b}$.

For supersonic jet Mach numbers of 1.2 and 1.4 the peak impact pressure fluctuations increased for the 2 in. nozzle, cf. Fig. Ila, and the location of the peak fluctuations was in the vicinity of the sonic velocity on the axis. The shadowgraph photographs of the jet flows from a convergent nozzle operated over a Mach number range of 0.6 to 1.4 are shown in Ref. 6 and Fig. 12. For supersonic Mach numbers the photographs show the presence of shock bottles, but evidently the, impact pressure fluctuations at the normal shock waves in the bottles are not large compared to the fluctuations present at the end of the supersonic flow region. After the location of the peak fluctuations for supersonic jet Mach numbers, the fluctuations decreased as $x^{-1.74}$, similar to that observed with subsonic jets. Thus, the maximum impact pressure fluctuations for supersonic jets occur in the vicinity of the sonic velocity on the axis while for subsonic jets the peak fluctuations occurred at the end of the "adjustment" or mixing region of the jet.

The rms piezoelectric impact pressure fluctuations for the 1 in. convergent nozzle, Fig. 2, were obtained with the piezoelectric impact probe with an inlet opening of 0.055 in. as shown in Fig. 4. For subsonic jet Mach numbers of 0.6 to 1.0 , the variations of the piezoelectric impact pressure fluctuations along the axis were similar with the peak occurring at approximately 8 diameters from the nozzle exit6. Downstream of the peak location the impact pressure fluctuations decreased as $\mathrm{x}^{-1.74}$; similar to that observed with the 2 in. convergent nozzle, Fig. 1la. At supersonic Mach numbers of 1.2 and 1.4 the peak impact pressure fluctuations occurred in the vicinity of the sonic location. And downstream of the peak location in the fully developed subsonic turbulent flow region the impact pressure fluctuations decreased also as $x^{-1.74}$. The results for the impact pressure fluctuations along the axis for the 2 in. and 1 in. convergent nozzle indicate that for a fully developed subsonic flow region the rms impact pressure fluctuations decreased as $x^{-1.74}$ and the peak fluctuations occurred in the vicinity of the sonic. location for the supersonic jets. 


\subsection{Axial Piezoelectric Static Pressure Fluctuations}

The static pressure fluctuations in the subsonic and supersonic flows were investigated with various diameters and tip shapes for the static pressure probes with piezoelectric quartz pressure gage and $B \& K$ microphone for sensing the static pressures as shown in Fig. 4. Axial static pressure surveys were conducted for both 2 in. and 1 in. nozzles with the sharp tipped $1 / 8$ in. diameter piezoelectric static pressure probe for subsonic and supersonic jet flows. For subsonic flows both the sharp tipped and blunt tipped static pressure probes were utilized.

To correlate the piezoelectric impact pressure probe results, Fig. $11 a$, for the 2 in. convergent nozzle, an investigation was conducted with the sharp tipped $1 / 8$ in. diameter piezoelectric static pressure probe to investigate the static pressure fluctuations for jet Mach numbers of $0.60,1.0$, and 1.4 , and the results are presented in Fig. 13a. For subsonic jet Mach numbers of 0.6 and 1.0 , the rms piezoelectric static pressure fluctuations over the initial 5 diameters from the nozzle exit were nearly constant. As shown by Eq. (12) the piezoelectric probe output is dependent on the static pressure fluctuations due to turbulent velocity fluctuations and the acoustic waves present in the flow. At a jet Mach number of 0.6 the peak rms static pressure fluctuation occurred at approximately 9 diameters from the nozzle exit, and this was the location for the peak impact pressure fluctuation, Fig. lla. For sonic jet Mach number the location of the peak fluctuations occurred at approximately 11 diameters from the exit. The rms static pressure fluctuations decreased continuously from the peak location for both subsonic Mach numbers in the fully developed subsonic turbulent flow region. At a jet Mach number of 1.4 the peak rms static pressure fluctuations occurred ahead of the sonic location on the axis, and the peak level was nearly an order of magnitude greater than for the sonic jet. Again, downstream of the sonic location the magnitude of the static pressure fluctuations decreased in the fully developed subsonic flow region like the subsonic exhaust velocities.

Similar axial piezoelectric static pressure probe surveys ${ }^{9}$ were conducted with the sharp tipped small probe for the 1 in. diameter convergent nozzle, Fig. 2, over a jet exhaust Mach number range of 0.6 to 1.4 and the results are presented in Fig. $13 \mathrm{~b}$. The rms static pressure fluctuations near the nozzle exit for jet Mach numbers of $0.6,0.7$, and 1.0 were quite close. In the fully developed subsonic turbulent flow region for these jet Mach numbers, which corresponds to a distance greater than approximately 10 diameters from the nozzle exit, the rms static pressure fluctuations decreased approximately as $(x / D)-1.95$. At supersonic jet Mach numbers of 1.2 and 1.4 , the peak static pressure fluctuations 
occurred ahead of the location for the sonic velocity on the jet axis. And the magnitudes of these peak pressure fluctuations were much greater than the peak value observed for the sonic jet. For both of these supersonic jet Mach numbers the rms piezoelectric static pressure fluctuations decreased from the peak value monotonically with distance in the subsonic turbulent flow region. The rate of decrease of the static pressure fluctuations with distance was similar to those observed for the subsonic jet exhaust velocities.

The axial piezoelectric static pressure probe data for the 1 in. nozzle was plotted in Fig. $13 \mathrm{c}$ as a function of the local flow Mach number by the use of the velocity variation with distance presented in Fig. llb. The rms static pressure fluctuations were plotted in this manner to estimate the magnitude of the acoustic pressure fluctuations present in the jet plume. It was shown in Eq. (12) that for a subsonic turbulent flow the static pressure fluctuation was dependent upon the static pressure fluctuations due to turbulence, Eqs. (8) or (10), and the acoustic waves. On the axis of the subsonic jet the radial velocity gradient is equal to zero so that Eq. (8) is applicable for determining the static pressure fluctuation caused by turbulence. Thus, on the axis for the same local flow velocity the static pressure fluctuations produced by turbulence should be the same. And the difference in the static pressure fluctuations must be due to the acoustic pressure fluctuations. By comparing the rms static pressure fluctuations for a local Mach number of 0.4 on the axis, there is a noticeable higher static pressure fluctuation for a jet Mach number of unity than for a jet Mach number of 0.6 as shown in Fig. 13c. At higher jet Mach numbers of 1.2 and 1.4 the local static pressure fluctuations on the axis were higher in the subsonic flow region. The acoustic waves produced by the shock bottles for the supersonic jet velocities and by the higher turbulent velocities seem to become significant and are propagated through the jet plume.

\subsection{Contours of Mach Number, Piezoelectric Impact and Static Pressure Fluctuations.}

To determine the distributions of the Mach number, piezoelectric impact and static pressure fluctuations in the jet plume for subsonic and supersonic jet Mach numbers, detailed surveys of the jet plumes were conducted with the small pitot tube, piezoelectric impact and static pressure probes, cf. Fig. 4, for the 1 in. diameter convergent nozzle, Fig. 2, and some of the results for these investigations 6,9 are presented in Figs. 14 and 15 . From the pitot tube surveys of the jet plume and assuming that the static pressure in the jet was equal to the ambient pressure the local flow Mach numbers were determined for jet Mach numbers of 0.6 to 1.4 in Ref. 6, and the contours of the constant Mach numbers of 1.0 and 1.4 are presented in Fig. 14.. For subsonic jet Mach 
numbers of 0.6 to 1.0 the constant Mach number contours were similar to that observed for the jet Mach number of unity ${ }^{6}$. The mixing of the ambient air with the sonic jet was rather rapid and the contour for a local flow Mach number of 0.2 moved out radially rather slowly with distance. For a jet Mach number of 1.4 the sonic velocity on the axis was located approximately 10 diameters from the exit of the convergent nozzle as shown in Fig. 14. The subsonic mixing region surrounding the supersonic jet exhaust increased very rapidily with distance as the ambient air was entrained by the supersonic velocity jet. Thus, at the end of the supersonic region on the axis the jet diameter was nearly 1.8 times the nozzle exit diameter. The acoustic radiation from the supersonic mixing region of the Mach 1.4 jet is surrounded by a rather thick subsonic mixing region.

The contours of constant rms piezoelectric impact and static pressure fluctuations in the flow field for a 1 in. diameter convergent. nozzle at Mach numbers of 1.0 and 1.4 are presented in Figs. 15a and 15b. The contours of constant rms piezoelectric impact pressure fluctuations were determined in Ref. 6 over a jet Mach number range of 0.6 to 1.4 . For subsonic jet Mach numbers of 0.6 to 1.0 the rms piezoelectric impact pressure contours were quite similar to the contours observed for a Mach 1.0 jet, presented in Fig. 15 . The peak impact pressure fluctuations were observed in a toroidal region located over axial locations of approximately 4 to 6 diameters from the nozzle exit and with the center located off the axis. It was shown by Eq. (7) that the output of the piezoelectric impact pressure was a function of the local mean density and velocity and the rms turbulent velocity.

With the sharp tipped $1 / 8$ in. diameter piezoelectric static pressure probe, Fig. 4, the flow field of the 1 in. diameter convergent nozzle was surveyed over jet Mach numbers of. 0.6 to 1.4 in Ref. 9 . The static pressure fluctuation contours for a jet Mach number of unity are presented in Fig. 15a. Again the static pressure fluctuations contours for jet Mach numbers of 0.6 to 1.0 were similar as observed with the piezoelectric impact pressure contours. The peak static pressure fluctuations occurred near the nozzle exit in the mixing region. For a given axial location the maximum rms static pressure fluctuations occurred at radial positions slightly larger than the nozzle radius, and the radial location of the peak pressure fluctuations moved farther out radially with distance. In Fig. 15a the outer boundary of the jet plume is indicated by the dashed line. It is interesting to note that the static pressure fluctuations are present outside the jet plume since the piezoelectric static pressure output is dependent upon the acoustic pressure and the static pressure fluctuations due to turbulence. Outside the jet plume the turbulent static pressure fluctuations do not exist because the turbulent velocity fluctuation is equal 
to zero, and consequently, Eq. (12) for the static pressure probe output indicates the existence of acoustic pressure outside the jet plume.

Similar piezoelectric impact and static pressure surveys were conducted with the 1 in. nozzle at a Mach number of 1.4 , and the results are presented in Fig. $15 \mathrm{~b}$ and Refs. 6 and 9. The contours for the rms piezoelectric impact pressure fluctuations are entirely different than the contours for the rms piezoelectric static pressure fluctuations. At a given axial location the peak impact pressure fluctuation occurred in the vicinity of the sonic velocity contour, and the maximum piezoelectric impact pressure fluctuations occurred at the end of the supersonic region, approximately 9 diameters from the nozzle exit. The maximum static pressure fluctuations occurred in the vicinity of the end of the supersonic region for a. Mach 1.4 jet as shown in Fig. 15b. The static pressure contours at this Mach number are entirely different than the contours observed for the sonic jet Mach number, Fig. 15a. For supersonic Mach number the rms piezoelectric static pressure fluctuations were quite large outside the jet plume and this rms pressure was due to the acoustic waves. Similar rms piezoelectric static pressure fluctuation contours were observed for a supersonic jet Mach number of 1.2 . 


\subsection{ACOUSTIC CHARACTERISTICS OF SUBSONIC AND SUPERSONIC JETS}

6.1 Flow and Acoustic. Characteristics of Subsonic and Supersonic

The available references on the flow and acoustic characteristics for subsonic and supersonic jets have indicated that both flow and acoustic phenomena do change from subsonic to supersonic jet velocities $3-6,9,10-21$, and the main features of subsonic and supersonic jets are characterized in Figs. 16 and 17. For subsonic jet velocities including sonic jet, the core region extends over the initial 4 to 5 diameters from the jet exit, and the length depends upon the initial turbulence level in the flow $4,6,10-12$. Downstream of the core region the adjustment region occurs with large turbulence before the jet becomes fully developed turbulent flow as shown in Fig. 16. In this fully developed turbulent flow the velocity decreases as $x^{-1}$ and the piezoelectric impact pressure fluctuations decays as $\mathrm{x}^{-1.74}$ as observed in Refs. 4, 6, and 9 .

For a properly expanded supersonic jet through a convergent-divergent nozzle, the velocity at the nozzle exit will be uniform and parallel to the axis with the static pressure equal to the ambient pressure, and this type of supersonic flow is indicated in Fig. 17. There will be a supersonic core region surrounded by supersonic and subsonic mixing regions, and downstream of the supersonic region the jet flow becomes fully developed turbulent subsonic flow with velocity decaying as $x^{-1}$ and piezoelectric impact pressure fluctuations decaying as $x^{-1.74}$ as observed in Refs. 4, 6, and 9. It was observed in Ref. 3 and seen in Fig. 18 that the core and supersonic lengths were primarily functions of the jet Mach number, and that the size of the nozzle and the temperature of the jet for supersonic velocities were second order effects as discussed in Refs. 3 and 5. Over the supersonic portion of the jet, Potter and Jones ${ }^{16}$ determined the acoustic radiation distribution to increase nearly linearly from the Mach 2.4 jet exit to the sonic location as shown in Fig. 19a, and Nagamatsu, sheer, and Gill4 observed similar results for a Mach 1.4 jet from the near field microphone measurements as shown in Fig. 19b. Downstream of the supersonic region in the fully developed subsonic turbulent flow the acoustic radiation per unit length of the jet decreased as $x^{-6}$, which was postulated by Lighthilil7 for a subsonic jet.

6.2 Jet Noise Theories

6.2.1 Subsonic Jet. Noise Theory of Lighthill

Lighthill 17 derived the equation for the overall sound power for subsonic jets based upon the dimensional analysis and experimental acoustic data as 


$$
W=10^{-4} \frac{1}{2} \rho_{a} \frac{U_{j}^{8} A}{c_{a}^{5}},
$$

in which the jet density $\rho_{j}$ was equal to the ambient density $\rho_{p}$. To account for the differences in the physical state of the jel with that of the ambient gas, Eq. (19) can be expressed as

$$
W=10^{-4} \frac{m}{2} \frac{\rho_{j}}{\rho_{a}}\left(\frac{c_{j}}{c_{a}}\right)^{5} \cdot c_{j}^{2} M_{j}^{7}
$$

where $m=\rho_{j} U_{j} A$ is the mass flow of the jet. By assuming the sound emitted from the mixing region, $0 \leq \mathrm{x} \leq 4 \mathrm{D}$, to be constant and the fully developed turbulent decay region as $4 \mathrm{D} \leq \mathrm{x}<\infty$, cf. Fig. 16, the overall acoustic power output can be expressed by

$$
W=\int_{0}^{4 D} w_{m} d x+\int_{4 D}^{\infty} w_{m}(4 D)^{6} x^{-6} d x
$$

where $w_{m}$ is the sound emission per unit length in the mixing region. As shown in Refs. 3 and 5 the acoustic power output per unit diameter length in the mixing and turbulent decay regions can be expressed as 22

$$
\begin{gathered}
w_{d_{m}}=\frac{10^{-4}}{9.6} \frac{\rho_{j}}{\rho_{a}} m\left(\frac{c_{j}}{c_{a}}{ }^{5} c_{j}^{2} M_{j}^{7},\right. \\
0 \leq x / D \leq 4
\end{gathered}
$$

and

$$
\begin{aligned}
w_{d}= & \frac{10^{-4}}{9.6\left(4^{-6}\right)} \frac{\rho_{j}}{\rho_{a}} m\left(\frac{C_{j}}{c_{a}}\right)^{5} c_{j}^{2} M_{j}^{7} \\
& \leq \leq x / D \leq \infty
\end{aligned}
$$

\section{2. 2Su Supersonicejet Noise Theory oif Nagamat sutand. Horvay}

The acoustic radiation from supersonic jets has been analyzed by various investigators $3,5,23-26,42,43$. An analysis of the acoustic power output for fully expanded supersonic jets was made by 
Nagamatsu and Horvay ${ }^{3}, 5$ by considering the experimental flow and acoustic characteristics. From the available data for jets with various diameters and temperatures it was found that the supersonic core length and the length of the supersonic region were a function of jet Mach number as shown in Fig. 18, and the supersonic length was derived in Ref. 3 as

$$
L_{s}=\frac{l^{l} s}{D}=5 M_{j}^{2}+0.8
$$

Also, in the supersonic region the acoustic emission per unit length was found to vary almost linearly with distance by Potter and Jones 16 at a Mach number of 2.49, Fig. 19a, and by Nagamatsu, Sheer and $\mathrm{Gill}^{4}$ for a. Mach 1.4 jet, Fig. 19b. The acoustic radiation at these supersonic Mach numbers occurred at the end of the supersonic region on the axis, and downstream of this peak acoustic radiation location in the subsonic turbulent flow the acoustic radiation per unit length decreases as $x^{-6}$.

Using these flow and acoustic features of supersonic jets, Nagamatsu and Horvay derived an equation for the overall acoustic power output for supersonic jets in Refs. 3 and 5 . The overall sound power output from a supersonic jet can be expressed as a sum of the acoustic contribution from the supersonic region $0 \leq x \leq l_{s}$ ! and the subsonic turbulent decay region, $l_{s} \leq x<\infty$, by

$$
w=\int_{0}^{\ell} w d x+\int_{l_{s}}^{\infty} w d x
$$

where $w$ is the acoustic power output of a jet slice of unit length and is a function of $x$ for a given jet condition. This may be written with $w_{d}=w_{j}$ as

$$
W=\int_{0}^{L_{s}} w_{d} d(x / D)+\int_{L_{s}}^{\infty} w_{d} d(x / D)
$$

It is shown in Ref. 3 that the acoustic power output per unit diameter length is given in the supersonic region, $0 \leq x / D \leq I_{s}$ by 


$$
w_{d}=\frac{10^{-4}}{9.6} m \frac{\rho_{j}}{\rho_{a}}\left(\frac{c_{j}}{c_{a}}\right)^{5}\left(c_{j}^{2} M_{j}^{7-\alpha}\right)\left(1+\frac{M_{j}^{\alpha-\beta}-1}{5 M_{j}^{2}+0.8}\right)(x / D)
$$

and in the subsonic region, $I_{S} \leq x / D<\infty$, by

$$
w_{d}=\frac{10^{-4}}{9.6} m \frac{\rho_{j}}{\rho_{a}}\left(\frac{C_{j}}{c_{a}}\right)^{5}\left(c_{j}^{2} M_{j}^{7-\beta}\right)\left(5 M_{j}^{2}+0.8\right)^{6}(x / D)^{-6}
$$

where $\alpha$ and $\beta$ are exponents which must be evaluated from experimental acoustic data as presented in Ref. 4. Substituting Eqs. (25) and (26) into Eq. (24a), the total acoustic power output from a supersonic jet is given by

$$
\begin{gathered}
w=\frac{10^{-4}}{9.6} m \frac{\rho_{j}}{\rho_{a}}\left(\frac{c_{j}}{c_{a}}\right)^{5}\left(c_{j}^{2} M_{j}^{2}\right)\left(5 M_{j}^{2}+0.8\right) \\
\left(\frac{M_{j}^{-\alpha}+M_{j}^{-\beta}}{2}+\frac{M_{j}^{-\beta}}{5}\right)
\end{gathered}
$$

These equations derived by Nagamatsu and Horvay were used to analyze the sound emission from supersonic jets.

Nagamatsu and Horvay in Ref. 3 solved these equations for the acoustic radiation per unit length and the overall sound power levels for supersonic jets by using the acoustic distribution observed by Potter and Jones 16 for a Mach 2.49 jet to determine the values of $\alpha$ and $\beta$ of 6.2 and 2.4 respectively. Using these values as a first approximation in Eqs. (25) and (26) the acoustic power generated per unit length of jet for various jet Mach numbers were calculated, and the results for jet Mach numbers of 1.0 and 1.5 are presented in Figs. $20 \mathrm{a}$ and $20 \mathrm{~b}$ respectively for various total temperatures for the jet exhaust. For sonic exhaust velocity, Fig. 20a, the acoustic emission per unit length for unit slug mass is constant over the initial 5.8 diameters before decreasing for the jet temperature range of $520^{\circ}$ to $3500^{\circ} \mathrm{R}$. The effects of the jet temperature is to increase the level of the acoustic radiation because of the increased jet velocity as shown by Eq. (25). For a jet Mach number of 1.5 the calculated acoustic 
radiation from Eqs. (25) and (26) indicated that the radiation increased from the nozzle exit to a peak value located at the sonic location on the axis, 12 diameters from the nozzle, as shown in Fig. $20 \mathrm{~b}$.

The overall sound power levels were calculated from Eq. (27) with values of $\alpha$ and $\beta$ of 6.2 and 2.4 respectively determined from the Mach 2.49 jet as a first approximation. In Fig. 21 a the overall sound power levels are presented as a function of the jet Mach number for jet temperatures of $520^{\circ}$ to $3500^{\circ} \mathrm{F}$ and unit slug mass. The overall acoustic power level increased with the total jet temperature for a given jet Mach number, and the curves for the overall sound power levels at constant jet temperatures were similar. The slopes of these overall sound power level curves decreased as the jet Mach number increased. In Fig. 2lb the overall sound power levels calculated from Eq. (27) are presented as a function of the jet exhaust velocity for jet temperatures of $520^{\circ}$ to $3500^{\circ} \mathrm{R}$ for unit slug mass. The slope of the overall sound power level was the greatest for a jet temperature of $520^{\circ} \mathrm{R}$ and the slope decreased with the increase in the jet temperature. And this decrease in the slope is caused mainly by the decrease in the jet density, $\rho_{j}$, with increasing jet temperature in Eq. (27). The overall sound power levels obtained with room temperature jets in Ref. 4, and at approximately $2000^{\circ} \mathrm{R}$, in Ref. 44 are presented in Fig. 2lb. The slope of the experimental data for the overall sound power level curves decreased with jet temperature, similar to the decrease given by Eq. (27).

\subsection{Near-Field Acoustic Characteristics}

\subsubsection{Near-Field Pressure: Fluctuations}

The near-field acoustic characteristics of subsonic and supersonic jets were investigated by placing two microphones, one on each side of the jet at various radial distanges and moving these microphones axially from the nozzle exit. These microphones were placed at radial positions of 2 to 8 diameters from the periphery of the convergent nozzle exit for jet Mach numbers of 0.6 to 1.4 , and the results for the microphone placed at radial distances of 2 and 4 diameters are presented in Fig. 22: These near-field measurements were made, similar to the experiments by Lassiter and Hubbard10, for the purposes of determining the near-field acoustic characteristics of subsonic and supersonic jets as well as to obtain information regarding the acoustic power transmitted through a cylindrical surface surrounding the subsonic and supersonic jets.

For subsonic jet Mach numbers of 0.6 to 1.0 the variations of the sound pressure level, for microphones placed radially at 2 and 4 diameters from the nozzle periphery, with axial distance were 
quite similar, Fig. 22, indicating the same type of acoustic radiation from subsonic jets, as well as for a sonic jet. The greatest increase in the sound pressure fluctuations with axial distance occurred with the microphone at the 2 diameter location. And the least variation of the sound pressure level with distance occurred with microphone eight diameters away from the nozzle for the subsonic Mach numbers. The axial sound pressure level variations for microphones placed at 2 and 4 diameters from the nozzle exit for supersonic jet Mach numbers of 1.2 and 1.4 were quite different than those observed for sonic and subsonic jets as indicated in Fig. 22. At the nozzle exit the sound pressure levels increased drastically compared to the value for the sonic jet. This increase in the sound pressure. level must be caused by the presence of Mach waves and supersonic radiation as indicated by the optical results, Fig. 12. The increase in the sound pressure levels with distance for the radial distances of 2 and 4 diameters at Mach numbers of 1.2 and 1.4 was much less than that observed for subsonic Mach numbers. These near-field acoustic results for the supersonic jet Mach numbers indicate that the mechanism of acoustic radiation from supersonic jets is different from that existing for the sonic and subsonic jets, thus, the subsonic jet noise theories of Lighthill17 and others 22 do not apply, and improved theory for the supersonic jet noise must contain the correct flow and acoustic features of supersonic jets as developed by Nagamatsu and Horvay ${ }^{3}$.

\subsubsection{Distribution of Acoustic Power Emission}

From the near-field sound pressure measurements with microphones, the acoustic intensity and the acoustic power transmitted through a cylindrical surface containing the microphone were calculated for subsonic and supersonic jets. The intensity of acoustic radiation at the microphone location is given by

$$
I=\frac{\bar{p}^{2}}{\rho_{a} c_{a}}
$$

where $\bar{p}$ is the rms sound pressure. By assuming that the sound emission from the circular jet is axially symmetric, the sound. power transmission per unit length through a cylindrical surface containing the microphone is

$$
\ddot{w}=\int_{0}^{2 \pi} \frac{\bar{p}^{2}}{\rho_{a} c_{a}} r d \theta
$$

and in terms of the unit jet diameter length 


$$
w_{d}=\frac{D}{\rho_{a} c_{a}} \int_{0}^{2 \pi} \bar{p}^{2} r d \theta
$$

Using these equations the acoustic power transmission through the cylindrical surfaces were calculated and the results for jet Mach nmbers of 1.2 and 1.4 are presented in Fig. 19b. Similar results for sonic and subsonic jet Mach numbers and various radial locations for the microphones are presented in Refs. 3 and 5 . The variations of the acoustic power transmission per unit length in the axial direction with the microphone at a radial location of 2 diameters are not continuous but can be approximated by a linear variation up to the sonic location. To obtain some information regarding the acoustic power radiation from a convergent nozzle, the acoustic power distributions presented in Fig. 19b were integrated to obtain the overall acoustic power level by

$$
W=\int_{0}^{\ell_{C}} w 2 \pi r d x+\int_{\ell_{C}}^{\infty} w 2 \pi r \ell_{c}^{6} x^{-6} d x
$$

in which the acoustic radiation per unit length was assumed to decay as $x^{-6}$ and $l_{C}$ is the core length for the subsonic jets and $l_{s}$ the supersonic length is used for the supersonic jets. Using this equation the overall acoustic powers were calculated for subsonic and supersonic jet Mach numbers and the results for Mach 1.2 and 1.4 jets are presented in Fig. 19b. Also, in this figure the overall sound power levels determined from far-field microphone measurements are presented. For the Mach 1.2 jet the overall sound power levels determined from near- and far-field measurements were 149 and $148 \mathrm{db}$ respectively, while the corresponding results for the Mach 1.4 jet were 158 and $156 \mathrm{db}$. The agreements between the near-field integrated overall sound power level and that from the far-field measurements are surprisingly close. For subsonic jet Mach numbers of 0.85 and 1.0 the agreement between the near- and far-field results was not as good in Ref. 4. The excellent agreement for the supersonic Mach numbers may be due to the existence of the Mach waves for supersonic jets as shown in Refs. 6, 16 , and 18. Thus, in the near-field location the microphone will be influenced primarily by the Mach waves and sound waves from the region of the jet upstream of the microphone location. And sound emission from the region of the jet downstream of the microphone will be highly attenuated or will not reach the microphone because of the supersonic jet velocity. 


\subsection{Sound Pressure Level Distribution}

The overall sound pressure levels were determined from the microphone measurements on a 10-ft. radius from the jet axis as shown in Fig. 1. Eight angular positions from the axis of $19.1^{\circ}$ to $146.4^{\circ}$ were used to determine the overall sound pressure levels ${ }^{4}$, and the results for jet Mach numbers of 0.60 to 1.4 are presented in Fig. 23. For subsonic jet velocities the variations of the overall sound pressure level with angular position were quite similar with the maximum pressure level at an angular position of $19.1^{\circ}$ and the pressure monotonically decreased with increasing angle. Similar variations of the overall sound pressure level with angular position were observed by other investigatorsll-15 with room temperature jets.

For supersonic jet Mach numbers of 1.2 and 1.4 the far-field sound pressure level. distributions are different from those observed for subsonic jets as shown in Fig. 23. The maximum overall sound pressure levels were located close to the jet axis for supersonic Mach numbers and decreased to $43.8^{\circ}$ location. Beyond this angular position the sound intensity remained nearly constant for the jet Mach number of 1.4. This type of sound pressure level variation is due to the occurrence of eight shock bottles, Fig. 12, at this Mach number with corresponding large acoustic radiation from each bottle as discussed in Ref. 18. When the supersonic jet is perfectly expanded through a contoured nozzle to the ambient air, there are no shock bottles as shown in Refs. 16, 19, and 20. In Ref. 2 it was observed that the overall sound power level decreased with angular position from the jet axis for a convergent-divergent nozzle designed for a Mach number of 1.5 .

\subsection{Sound Power Spectra and Overall Sound Power Level}

\subsubsection{Sound Power Spectra}

From the microphone measurements at eight angular positions on a radius of 10-ft. from the 2 in. diameter convergent nozzle exit, Fig. 1, the spectra of the acoustic power output per $1 / 3$ octave frequency band for jet Mach numbers of 0.60 to 1.40 were obtained 4 and are presented in Fig. 24. The power spectra was obtained over a frequency range of $40 \mathrm{~Hz}$ to $16 \mathrm{KHz}$ with the available tape recorder. Since the microphone was on a lo-ft. radius, the sound power levels below $100 \mathrm{~Hz}$ were obtained for wave lengths greater than $10 \mathrm{ft}$, which caused the scatter below $100 \mathrm{~Hz}$. The sound power spectra for jet Mach numbers of 0.60 to 1.0 were quite similar with peak power occurring at about the same frequency. At the highest Mach number of 1.4 , the maximum power occurred at a higher frequency than that for the subsonic jets. Acoustic data for the Mach 1.4 jet was obtained also with a tape recorder with 
response up to $80 \mathrm{KHz}$ and the power spectrum from this data agreed with that observed with the tape recorder over a frequency range of $40 \mathrm{~Hz}$ to $16 \mathrm{kHz}$. For frequencies greater than $16 \mathrm{kHz}$ the power continuously decreased indicating that nearly all of the acoustic power is contained in frequencies below $16 \mathrm{KHz}$.

\subsubsection{Overall Sound Power Level}

In Ref. 4 the overall sound power levels were determined for the 2 in. diameter convergent nozzle operated over a Mach number range of 0.60 . to 1.4 . Since the mass flow of the nozzle decreased for lower Mach numbers from that existing at a Mach number of 1.4, the overall sound power levels were corrected to the Mach 1.4 mass flow rate of $0.1036 \mathrm{slugs} / \mathrm{sec}$. This was the procedure used to obtain the variation of the overall acoustic power with jet velocity for constant mass flow rate in Ref. 6 and the results are presented in Fig. 25. Over the subsonic velocity range of 672 to $1038 \mathrm{ft} / \mathrm{sec}$., which correspond to jet Mach numbers of 0.6 to 1.0 , the overall acoustic power increases approximately as us. And for the higher velocity range of 1038 to $1346 \mathrm{ft} / \mathrm{sec}$., Machnnumber of t 0 to 1.4 , the overall acoustic power increased approximately as $u_{j} 2$. There is a discontinuity in the slope of the overall acoustic power variation with velocity for subsonic and supersonic jet velocities. This variation is different than that predicted by Lighthill17 and others 22,25 in which these authors predicted a decrease in the slope of the overall acoustic power with velocity.

The increase in the slope of the overall sound power with velocity for the supersonic Mach numbers is caused primarily by the presence of the shock bottles with a convergent nozzle, Fig. 12 . Existing jet noise theories of Lighthilil7 and Ribner ${ }^{2}$ were derived on the acoustic radiation from turbulence but the noise generation from shock waves was not considered in these theories. But the supersonic jet noise theory of Nagamatsu and Horvay ${ }^{3}$ was based upon the flow and acoustic characteristics of supersonic jets. The effects of Mach number and shock waves were included in the theory by the introduction of the parameters $\alpha$ and $\beta$, and these values for convergent and parallel flow nozzles were determined for room temperaturesjets as functions of the jet Mach number in Ref. 5 and are presented in Fig. 26.

6.6 Sound Pressure Level Spectra as Function of Angular Position and Mach Number

The $1 / 3$ octave band sound pressure level spectra were determined for the 2 in. convergent nozzle from the microphone measurements at eight angular positions, and the results are presented in Figs. $27 \mathrm{a}$ and $27 \mathrm{~b}$ for jet Mach numbers of 1.0 and 1.40 respectively. Again in these figures the sound pressure levels for frequencies 
less than $100 \mathrm{~Hz}$ are not reliable because the microphone data were obtained on a 10-ft. radius. For sonic jet Mach number the sound pressure levels at the $19.1^{\circ}$ position increased to a peak value at approximately $1.1 \mathrm{KHz}$ before decreasing, and over the frequency range of $70 \mathrm{~Hz}$ to $1.55 \mathrm{KHz}$ the sound pressure levels were the highest compared to other angular positions, Fig. 27a. At other angular positions the sound pressure levels over the frequency, range of $80 \mathrm{~Hz}$ to $3 \mathrm{KHz}$ decreased with increasing angles from the jet axis, with the lowest sound pressure levels observed for this frequency range at the largest angular location, $146.4^{\circ}$. Over the frequency range of $3 \mathrm{KHz}$ to $16 \mathrm{KHz}$ the sound pressure levels for all of the angular positions approached each other to within $5 \mathrm{db}$ at $16 \mathrm{KHz}$. Also, all the sound pressure level spectra varied continuously with frequency without any sharp discontinuity.

For a supersonic jet Mach number of 1.4 the $1 / 3$ octave band sound pressure levels are presented in Fig. $27 \mathrm{~b}$, and the sound pressure level spectra below $100 \mathrm{~Hz}$ are not reliable because microphone data were obtained on a 10-ft. radius ${ }^{2}$. At the $19.1^{\circ}$ location from the jet axis the sound pressure level increased to a peak value at approximately $2.7 \mathrm{KHz}$ before decreasing, and over the frequency range of $100 \mathrm{~Hz}$ to $2.7 \mathrm{KHz}$ the sound pressure levels were the highest compared to the other angular positions. For the $43.8^{\circ}$ position the sound pressure level increased from $100 \mathrm{~Hz}$ to $2.5 \mathrm{KHz}$, and over the frequency range of 2.5 to $16 \mathrm{KHz}$ the sound pressure level remained nearly constant. At the $60^{\circ}$ angular position the sound pressure level increasedccontinuously from $100 \mathrm{~Hz}$ to $1 \mathrm{l} \mathrm{KHz}$ before decreasing, and there is a rather rapid increase in the sound pressure level over the frequency range of $5 \mathrm{KHz}$ to $10 \mathrm{KHz}$, which is caused by the acoustic radiation from the shock bottles. Sonic jet Mach number sound pressure level spectra did not indicate this sudden increase in the sound pressure level at this frequency range, Fig. 27a.

At an angular position of $80.4^{\circ}$ the sound pressure level increased from $100 \mathrm{~Hz}$ to $6.4 \mathrm{KHz}$ before decreasing at higher frequencies as shown in Fig. 27b. The sound pressure level increased from $100 \mathrm{~Hz}$ to $3 \mathrm{KHz}$ for the $99.6^{\circ}$ location and the sound pressure level increased rapidly from $105 \mathrm{db}$ at $3 \mathrm{KHz}$ to a peak of $117 \mathrm{db}$ at 4.5 $\mathrm{KHz}$. This rapid increase in the sound pressure level over the frequency range of 3 to $4.5 \mathrm{KHz}$ could be caused mainly by acoustic radiation from the shock bottles. For the $120^{\circ}$ location the rapid increase in the sound pressure level occurred over the frequency range of 2 to $4 \mathrm{KHz}$, and for the $146.4^{\circ}$ location the sound pressure level increased rapidly over. the frequency range of 1.5 to $3.3 \mathrm{KHz}$. These sound pressure level spectra indicate the large increase in the sound pressure levels over the angular positions of $60^{\circ}$ to $146.4^{\circ}$. 
7.0 MULTIPLE TUBE AND SINGLE AND MULTIPLE SHROUD SUPPRESSORS FOR SUPERSONIC JETS

\subsection{Single Shroud with Six Rod Suppressor}

To obtain fundamental information regarding the supersonic jet noise suppression phenomenon, a long shroud was selected in Refs. 1 and 2 for inducing large amounts of ambient air and shielding the acoustic radiation from the supersonic region of the jet. This was accomplished by the use of a long 4 in. inside diameter pipe with a length of $58 \mathrm{in}$. to surround the 2 in. diameter convergent nozzle as shown in Fig. 28. Six 1/4 in. diameter rods were inserted into the periphery of the jet plume to produce shock waves which interacted with the shock bottles in the primary supersonic jet flow, Fig. 12. The leading edge of the shroud was located 3-1/4 in. upstream of the nozzle exit.

The convergent nozzle was operated at pressure ratios of 3.2 and 3.7 to achieve nominal jet Mach numbers of 1.4 and 1.5 . In Ref. 2 the axial impact Mach number distributions were determined for the various configurations of the nozzle, rods, and shroud at a jet Mach number of 1.5 and the results are presented in Fig. 29 . Because of the shock bottles in the jet plume, the Mach numbers determined from the impact pressure measurements varied over the initial portion of the supersonic jet. The flow became sonic at a location 31 in. from the nozzle exit. With the long shroud and with six rods inserted $1 / 4$ in. into the jet plume, the axial Mach number at the shroud exit was 0.78 and it remained nearly constant for 16 in. before decreasing slowly. with distance. With the entrance of the shroud sealed, Fig. 28, so there is no induced flow, the Mach number at the shroud exit was approximately 0.56 and the Mach number remained nearly constant over a distance of approximately 18 in. before decreasing with distance.

To obtain the overall sound pressure level distribution and the power spectra, the microphone was placed on a 10-ft. radius from the nozzle and shroud exits over angular positions of $19.1^{\circ}$ to $146.4^{\circ}$. The convergent nozzle was operated at a jet Mach number of 1.4 with and without the single shroud and six rods. For these conditions the overall sound pressure level distributions with angular position from the jet axis are presented in Fig. 29b. The overall sound pressure level for the plain nozzle was the highest at the $19.1^{\circ}$ position and decreased to approximately the $50^{\circ}$ location and remained nearly constant for larger angular positions. The single shroud with six rod configuration with induced flow decreased the overall sound pressure level $19.1 \mathrm{db}$ at the $19.1^{\circ}$ location from the value observed for the plain nozzle. And the sound pressure increased to a peak value at the $60^{\circ}$ position before decreasing at larger angular positions. At this location the sound 
pressure level was $8.4 \mathrm{db}$ less than the value observed with the convergent nozzle. With no induced flow through the shroud the sound pressure level at the $19.1^{\circ}$ location was decreased $13.7 \mathrm{db}$ from that observed with the plain nozzle. And the sound pressure level increased to a peak at the $60^{\circ}$ position before decreasing at larger angular positions. At this location the overall sound pressure level was slightly higher than the value existing with the plain nozzle. Similar results were observed in Ref. 2 for a jet Mach number of 1.5 .

The third-octave band sound power spectra for the convergent nozzle, shroud, and.six rod configurations for the jet Mach number of 1.4 are presented in Fig. 29c. For the plain nozzle the sound power level increased from $80 \mathrm{~Hz}$ to $4 \mathrm{KHz}$ before decreasing at higher frequencies. With the long shroud, six rods inserted $1 / 4$ in. into the jet plume, and induced mass flow of approximately 70 percent of the primary jet mass flow, the sound power spectra was lower than for the plain nozzle with the largest reduction of 22 $\mathrm{db}$ at $3.2 \mathrm{KHz}$. At $16 \mathrm{KHz}$ the reduction from the convergent nozzle was $11 \mathrm{db}$, and the overall sound power level reduction with this configuration was $14.2 \mathrm{db}$ from the convergent nozzle at a Mach 1.4 as shown in Fig. $29 \mathrm{c}$.

By sealing the entrance to the single shroud, no induced flow, the primary supersonic jet flow became subsonic within a few diameters from the nozzle exit as observed in Ref. 2 from the static pressure measurements along the shroud. There was a noticeable organ pipe effect present as indicated by the power spectra at $150 \mathrm{~Hz}$, Fig. 29c. Over the frequency range of $250 \mathrm{~Hz}$ to $8 \mathrm{KHz}$ the power spectra increased before reaching a peak and decreasing at $16 \mathrm{KHz}$. At 8 $\mathrm{KHz}$ the sound power level was approximately $3 \mathrm{db}$ lower than the value for the convergent nozzle. The Mach number at the exit of the shroud for this configuration was only 0.56, Fig. 29a, and the power spectra observed for a Mach 0.6 jet in Ref. 4 was much lower than that observed for the long shroud with no induced flow. The. overall sound power level was $147.5 \mathrm{db}$ which is only $4.5 \mathrm{db}$ lower than the value for the convergent nozzle. Without the shock waves in the shroud and for a jet Mach number of 0.56 the overall sound. power level reduction from a Mach 1.4 jet should be approximately $28 \mathrm{db}$ as discussed in Ref. 2. Therefore, the noise produced by shock waves in a long shroud propagates to the outside through a subsonic flow without appreciable attenuation.

\subsection{Tubes and 191 Shrouds Suppressor}

\subsubsection{Flow Characteristics}

Since the long shroud with rods suppressor configuration was effective in decreasing the Mach 1.4 jet by $14.2 \mathrm{db}$, Refs. 1, 2, and 
9, the multiple tube with multiple shroud suppressor configurations were investigated with Mach 1.4 jets in Refs. 7 and 8 . With the multiple tubes the sonic location from the tube exit was decreased drastically because the supersonic length was shown to be a function of the diameter for a given. Mach number as shown by Eq. (23). Thus, with the 191 tubes of 0.115 in. diameter, Fig. 30, the sonic location on the axis was located at approximately 1 in. as shown in Fig. 3lb, while the sonic location for the equivalent convergent nozzle diameter of 1-9/16 was approximately 20 in. from the exit at a jet Mach number of 1.4. By bringing the sonic location closer to the tube exit it is possible to make the multiple shroud length shorter compared to a nozzle with single shroud:

With the trolley system for the flow and acoustics facility, Fig. 1 , the variations of the flow field downstream of the multiple tubes and multiple shrouds were determined by the small impact pressure probe surveys in Ref. 7 and the Mach number profiles for the tube bundle with shrouds of various lengths are presented in Fig. 31a. One inch downstream of the 191 tubes the Mach number over the inner tubes varied from supersonic to subsonic velocities depending upon the location of the pitot tube relative to the tube exits. The velocity profile at the exit of the 191 shrouds with a length of 3.35 in. was much more uniform with mean Mach number of approximately 0.6 . By placing single shrouds of approximately 4 in. inside diameter and lengths of 3.8 and 12 in. at the exit of the tube bundle the Mach number profiles across the shroud exit were flat with mean Mach number of approximately 0.7 as shown in Fig. 3la. Other radial Mach number distributions at. the end of the single and multiple shrouds for a Mach 1.4 jet are presented in Ref. 7.

Impact pressure and total temperature surveys for the multiple tubes with and without multiple shrouds were made in the axial direction of the jets in Ref. 7. Using these measurements the local Mach number and velocity were calculated and the axial Mach number distributions for the 191 tubes with single and 191 shrouds are presented in Fig. 3lb. For the 1-9/16 in. diameter convergent nozzle at a nominal Mach 1.4, the local Mach number fluctuates in the supersonic region because of the shock bottles and the flow became sonic at approximately $20 \mathrm{in.}$ from the nozzle exit. With 191 tubes of $2 \mathrm{in}$. length the mixing of the supersonic jets with the ambient air was large with a corresponding decrease in the supersonic length. The merged Mach number downstream of the supersonic region was approximately 0.72 as indicated in Fig. 31b. This merged jet remained at this Mach number for about 16 in., which is approximately 4 diameters of the merged jet, before decreasing monotonically with distance. The addition of the 191 shrouds of 6 in. length to the multiple tubes decreased the supersonic jets to a Mach number of approximately 0.6 . Downstream of the uniform 
velocity region the velocity decayed very rapidly with distance, and this could be caused by the additional turbulence introduced by the 6 in. long multiple shrouds. The most drastic decrease in the velocity downstream of the 191 shrouds occurred for the 3.335 in. shroud length. With the single shroud at the exit of the tube bundle the axial Mach number at the shroud exit was approximately 0.68 , and the velocity decayed more rapidly with distance than for the 191 tubes.

The axial piezoelectric impact pressure fluctuations, determined with the quartz piezoelectric gage, for multiple tubes with and without the multiple shrouds were obtained in Ref. 7 , and the results for the multiple shrouds of various lengths and for a single shroud are presented in Fig. 3lc. The magnitude of the impact pressure fluctuations is presented in terms of rms values in millivolts. It was shown previously, Eq. (7), that for subsonic flows the output of the piezoelectric impact pressure is dependent upon the local mean density and velocity and the turbulence velocity fluctuation. For the plain nozzle at a Mach number of 1.4 , the impact pressure fluctuations remain low over the initial $15 \mathrm{in}$. before increasing rapidly to $4 \mathrm{mv}$ output at the 18 in. location before decreasing. This peak output is located just ahead of the sonic location on the axis as observed in Refs. 4, 6 , 8 , and 9.

For multiple tubes, the impact pressure fluctuations at 0.1 in. and $1.0 \mathrm{in.} \mathrm{is} 1 \mathrm{mv}, \mathrm{Fig}$. 3lc. It attains the lowest value of $0.13 \mathrm{mv}$ at the $5 \mathrm{in}$. location and then reaches the highest value of slightly over $1 \mathrm{mv}$ at the $30 \mathrm{in}$. location. Thus, the peak impact pressure fluctuations with the tubes is one-quarter of the peak value observed for the plain nozzle. Downstream of the location of the peak value for the tube, the piezoelectric impact pressure decreased at approximately $x^{-1.74}$ as observed for the plain nozzle in the fullyddeveloped subsonic turbulent flow, Fig. 11a. The magnitude of the peak piezoelectric impact pressure fluctuations for the multiple shroud lengths of $1.667,3.335$, and 6.0 in. decreased with the length as shown in Fig. 3lc, and the locations of the peak pressure fluctuations for these multiple shrouds were closer to the shroud exit. than for the multiple tube case. With the single shroud the peak value for the impact pressure fluctuation was close to the peak observed with the multiple tubes. For the tubes, multiple shrouds, and single shroud configurations, the impact pressure fluctuations downstream of the peak pressure location decreased very close to $\mathrm{x}^{-1.74}$ which was observed for the fully developed turbulent flow region. The variations of the impact pressure fluctuations with distance for multiple shrouds at various distances from the multiple tube exits are presented in Ref. 7 for a jet Mach number of 1.4 . 
The sound pressure level distributions for the multiple tubes and shrouds are presented in Fig. 31d as a function of the angular position from the jet axis. These overall sound pressure levels were determined from the microphones placed on a radius of 10-ft. from the suppressor exit. With the multiple tubes the sound pressure level at the angular position of $19.1^{\circ}$ was approximately 17 $a b$ less than the value for the plain nozzle, and the smallest difference of approximately $11 \mathrm{db}$ occurred at the $43.8^{\circ}$ location. At the $19.1^{\circ}$ position the decrease in the sound pressure level from that of the plain nozzle increased with the length of the multiple shrouds, and the reduction was nearly $25 \mathrm{db}$ for the 6 in. long shrouds. At the $60^{\circ}$ location the sound pressure level for the 6 in. long shrouds was approximately 18 db lower than the value for the plain nozzle, and at the $146.4^{\circ}$ position the sound pressure level reduction was about $25 \mathrm{db}$. The addition of the single shroud to the multiple tubes did not decrease the sound pressure levels from the levels for the multiple tubes. Additional sound pressure levels for various shroud configurations are presented in Ref. 7 .

The sound power spectra for the multiple tubes and multiple shrouds for a jet Mach number of 1.4 were obtained in Ref. 7 and the results for the multiple. shrouds of various lengths are presented in Fig. 31 e for a frequency range of $40 \mathrm{~Hz}$ to $16 \mathrm{KHz}$. The maximum sound power level of $145 \mathrm{db}$ occurs at $5 \mathrm{KHz}$ for the $1-9 / 16 \mathrm{in}$. diameter convergent nozzle at a Mach number of 1.4. At higher frequencies it decreases continuously and at $16 \mathrm{KHz}$ the sound. power level was $139 \mathrm{db}$. For multitubes the sound power level increases continuously from 75 to $1 \mathrm{KHz}$ and attains a value of 119.5 $\mathrm{db}$. The sound power level stays almost constant from 1 to $1.5 \mathrm{KHz}$, and it rises to $120 \mathrm{db}$ at $5 \mathrm{KHz}$ and drops to $119 \mathrm{db}$ at $6.3 \mathrm{KHz}$ before starting to rise at higher frequencies. As can be seen from Fig. 3le and Table $I$, the overall sound power level for multiple tubes is $135.5 \mathrm{db}$, which is a reduction of $15.2 \mathrm{db}$ from the plain jet at Mach number of 1.4. The sound power spectra are lowered by the addition of the multiple shrouds of various lengths over the frequency range of $75 \mathrm{~Hz}$ to $7 \mathrm{KHz}$ and at higher frequencies the 6 in. long shrouds decreased the power levels from the values existing with the multiple tubes. With this long shroud the sound power level of the Mach 1.4 jet was reduced by $20.4 \mathrm{db}$ forsthe ambient air temperature of $68^{\circ} \mathrm{F}$ as shown in Table I. The overall sound power level reduction for the Mach 1.4 jet increased with the length of the multiple shrouds. With the single shroud placed at the exit of the tubes the sound power spectra was higher than the plain tubes at higher frequencies, Fig. 3le, and the overall sound power level reduction was only $13.7 \mathrm{db}$ as shown in Table I, which is less noise suppression than the multiple tubes. Sound power spectra for other suppressor configurations for the convergent nozzle are presented in Ref. 7. 
To analyze the acoustic power output from the subsonic and supersonic regions for the multitubes, the Mach number distribution along the jet axis, Fig. 3lb, was used for a jet Mach number of 1.4. The jet flow coalesces at approximately $1-1 / 2$ in. from the tube exits, and the coalesced flow behaves like a uniform subsonic jet at a Mach number of approximately 0.72 and remains nearly constant for about 4 diameters before decreasing like a subsonic jet, Fig. 10. Using the mass flow determined 1 in. downstream of the tube bundie from the radial surveys in Ref. 7, the acoustic power output for this coalesced subsonic jet was calculated from Lighthil117 Eqs. (19a). The output power was calculated to be 0.75 watts or a sound power level of $128.8 \mathrm{db}$, which is less than the experimental value of $135.5 \mathrm{db}, 3.55$ watts, for the multiple tubes at a Mach number of 1.4 as shown in Table I. The difference in the acoustic power is contributed by the acoustic radiation from the supersonic region of the multiple tubes.

It is assumed that only the outer ring of 52 tubes are radiating acoustically from the supersonic region to the outside, with the acoustic radiation from the inner tubes assumed to be shielded by the outer tubes, the calculated power output from the supersonic jet noise theory of Nagamatsu and Horvay, Eq. (27) with $\alpha$ and $\beta$ determined from the convergent nozzle at a Mach number of 1.4 , Fig. 26 , is 19.6 watts or $142.9 \mathrm{db}$, which is much greater than the experimental value of $135.5 \mathrm{db}$. The difference between the acoustic power calculated for the subsonic region by Eq. (19a) and the experimental power is 2.80 watts. For each tube the calculated acoustic power output from supersonic region is 0.377 watts. Hence, it requires only 7.40 tubes to achieve 2.80 watts from the supersonic region, or approximately $51^{\circ}$ from each of the outer ring of 52 tubes. It is evident from this initial analysis of the acoustic data that the shielding and interference effects of the supersonic region from the 191 tubes are extremely large. Only the equivalent of about 4.3 percent of the 191 tubes are radiating acoustic power from the supersonic region to the surrounding air.

The multiple tube concept is effective as a supersonic jet exhaust noise suppressor because of the following effects: (1) with the smaller diameter tubes in place of a single nozzle the supersonic region is decreased, supersonic region is proportional to the diameter and the jet Mach number as shown in Ref. 3 and Eq. (23); (2) because of the larger exposed surface of the primary jet flow to the ambient air, the primary jet velocity is drastically decreased in a short distance and the coalesced jet behaves like a subsonic uniform jet; (3) finally the most effective contribution to the noise reduction is the large attenuation of the acoustic waves through the supersonic region, only a small fraction of the 
acoustic radiation from the supersonic region is propagated to the surroundings.

\subsubsection{Thrust Loss}

For the various configurations of the multiple tubes and multiple shrouds that were investigated, the change in the thrust from the equivalent convergent nozzle of $1-9 / 16$ in. diameter was measured with the delta-thrust device and is presented in Fig. $31 f$ and Ref. 7. The change in the thrust from the plain nozzle for the same reservoir and mass flow conditions is due to the skin friction in the 191 tubes and 191 shrouds and to the base pressure loss of the tube holder, cf. Fig. 30. An analysis was made to calculate the friction drag for the tubes and shrouds from the observed flow conditions and to determine the base drag from the measured base pressure distributions.

The flow through the 191 tubes was assumed to be sonic and uniform over the internal tube length of 2-1/4 in. Due to the boundary layer growth on the tube surface the sonic velocity will be attained at the end of the tube where the effective cross-sectional area is minimum. To estimate the friction drag in the 191 tubes, the skin friction for both laminar and turbulent boundary layers were calculated and compared with the experimental results. Since the flow in the tube is equal to or less than sonic, Blasius skin friction equation

$$
C_{f}=1.328 / \sqrt{R_{e}}
$$

was used for low subsonic Mach numbers and for $M=1.0$ the coefficient in this equation was decreased to 1.31 from the curve presented in Ref. 45. For turbulent boundary layer the empirical turbulent skin friction given by Schlichting 45 was used

$$
C_{f_{i}}=0.455 /\left(\log _{10} R_{e}\right)^{2.58}
$$

which agrees well with the experimental data for a Reynolds number range of $10^{6}$ to $10^{9}$.

Using these equations and the reservoir conditions for the Mach 1.4 jet flow condition, the skin friction drag was calculated for the multiple tubes for laminar and turbulent flows. Since, the Reynolds number based upon the tube length was $2.74 \times 10^{6}$, the flow in the tubes must be in the transition region because the 
measured friction drag was 8.2 percent of the thrust while the calculated values for laminar and turbulent skin frictions were 2.52 and 11.9 percent respectively as discussed in Ref. 7. The frictional drag of the multiple shrouds was calculated by using the velocity, temperature, and density determined from the radial surveys with the impact and total temperature probes at the shroud exit, Fig. 3la. From the mean values for the flow parameters at the multishroud exit, the Reynolds numbers were calculated for shroud lengths of 1.667 to 6.00 in. Because of the large disturbances in the mixing of the primary jet inside the shroud the boundary layer was assumed to be turbulent in the shroud passages, and the skin friction was calculated from Eq. (32) for the multiple shrouds. For a shroud length of 6 in. the skin friction thrust loss was $11.5 \%$ as shown in Ref. 7. The experimental thrust loss and the overall sound power level reduction of a Mach 1.4 jet is presented in Fig. 31 f as a function of the multiple shroud length. The thrust loss is appreciable for the 191 shrouds because of the large wetted surface exposed to the flow in the passages.

\subsection{Tubes and 191 Shrouds Suppressor for Mach 0.7 Jet}

\subsubsection{Flow Characteristics}

An investigation was conducted in Ref. 9 to determine the flow and acoustic characteristics of the 191 tubes and 191 shrouds for a subsonic jet exhaust Mach number of 0.7 , which corresponded to the exhaust Mach number for the GE-4 jet engine for the SST during cut-back for approach. In the earlier investigations of the multiple tubes and shrouds suppressor configurations in Ref. 7 , the acoustic characteristics of these suppressors at a jet Mach number of 0.7 were determined but no flow characteristics were obtained.

To obtain the flow information for these suppressors at a subsonic jet Mach number, axial surveys were conducted with an impact probe and piezoelectric impact and static pressure probes, Fig. 4, to obtain the mean and fluctuating velocities and fluctuating static pressures in Ref. 9. The 1-9/16 in. diameter convergent nozzle was operated at a pressure ratio across the nozzle of 1.4 to produce a jet Mach number of 0.7 , and the axial Mach number distributions for this condition is presented in Fig. $32 \mathrm{a}$ for various configurations. Over the initial 8 in. the flow Mach number is nearly constant, and the velocity decreases as $x^{-1}$ in the fully developed turbulent flow region. With the 191 tubes the jet coalesced at approximately 2 in. from the tube exits at a Mach number of 0.31 , and the Mach number remained nearly constant from this location to 20 in. downstream. For greater distances downstream the Mach number approached that for the plain nozzle as indicated in this figure. By adding multiple shrouds 
to the multiple tubes the Mach number at the shroud exit was approximately 0.28 and it decreased to 0.25 at the 2 in. location. The Mach number remained nearly constant to the location 12 in. from the shroud exit before decreasing rapidly with distance.

In Fig. $32 \mathrm{~b}$ the rms impact pressure fluctuations along the jet axis are presented for the convergent nozzle, multiple tubes, and multiple shrouds at a jet Mach number of 0.7 . The peak rms impact pressure fluctuation of $0.6 \mathrm{mv}$ occurred for the plain nozzle at a distance 14 in. from the nozzle exit. Downstream of this location the impact pressure fluctuations decreased as $x^{-1.74}$, which is the characteristics for a fully developed subsonic turbulent jet flow, Fig. 11. With the multiple tubes the peak impact pressure fluctuation of $0.14 \mathrm{mv}$ occurred at $30 \mathrm{in}$. from the tube exit before decreasing rapidly with distance. This peak rms pressure fluctuation is approximately $1 / 4$ of the peak value observed for the plain nozzle. For the multiple tubes and shrouds the peak rms impact pressure fluctuation of $0.078 \mathrm{mv}$ occurred at a location $18 \mathrm{in}$. from the shroud exit. Downstream of the peak location the rms impact pressure fluctuation decreased similar to that observed for the plain nozzle and multiple tubes.

The axial piezoelectric static pressure fluctuations for the various configurations at a jet Mach number of 0.7 were obtained with a sharp tip static pressure probe, Fig. 4, and the results are presented in Fig. 32c. For the convergent nozzle the rms static pressure fluctuations increased from the nozzle exit to a peak value of $0.066 \mathrm{mv}$ at $16 \mathrm{in.,}$ which is located 2 in. farther downstream than the location of the peak impact pressure fluctuation, Fig. $32 \mathrm{~b}$. Downstream of this peak location the static pressure fluctuations decreased slowly with distance to the 50 in. location, and beyond this point the static pressure fluctuations decreased more rapidly with distance. With the multiple tubes the peak rms static pressure fluctuation was $0.016 \mathrm{mv}$ at 25 and 35 in. from the tube exit. And this peak value is approximately $1 / 4$ of the peak rms static pressure fluctuation for the convergent nozzle. With the addition of the multiple shrouds to the tubes the peak rms static pressure fluctuation of $0.015 \mathrm{mv}$ occurred at 40 in. from the shroud exit. This peak value is close to the peak value for the multiple tubes even though the Mach number for the multiple shrouds is lower. than for the multiple tubes as shown in Fig. 32a. Evidently the large wetted surface in the multiple shrouds increased the viscous effects in the jet plume.

\subsubsection{Acoustic Characteristics}

The overall sound pressure levels as a function of the angular position from the jet axis for various configurations at a jet Mach number of 0.7 were determined in Ref. 9 and are presented in 
Fig. 32d. The microphone was placed on a 10-ft. radius from the jet exit. For the 1-9/16 in. diameter convergent nozzle the peak sound pressure level occurred at the $19.1^{\circ}$ location and it decreased monotonically with angular position as observed previously in Refs. 4,7 , and 8 for a, 2 in. diameter convergent nozzle of subsonic Mach numbers. With 191 tubes the peak sound pressure level increased from the $19.1^{\circ}$ location to a peak at $43.8^{\circ}$ before decreasing with angular position. At the 19.1' location the sound pressure level was approximately $9 \mathrm{db}$ less than that. observed for the plain nozzle. The addition of the multiple shrouds to the tubes decreased the sound pressure level at the $19.1^{\circ}$ position $13 \mathrm{db}$ from that observed for the plain nozzle. The sound pressure level increased to a peak value of $83.2 \mathrm{db}$ at approximately the $45^{\circ}$ location, and then decreased to the $80^{\circ}$ position after which it was nearly constant.

In Fig. 32ë the sound power spectra obtained in Ref. 9 for the convergent nozzle with various suppressor configurations are presented for a jet Mach number of 0.7. For the plain nozzle the peak power of $112.8 \mathrm{db}$ occurred at $4 \mathrm{kHz}$ and at higher frequencies the power level decreased continuously to $16 \mathrm{KHz}$ with an overall sound power of $120.5 \mathrm{db}$. With the 191 tubes the sound power level was less than that for the plain nozzle over the frequency range of $160 \mathrm{~Hz}$ to $16 \mathrm{KHz}$ with the greatest difference of approximately $15 \mathrm{db}$ from $1 \mathrm{KHz}$ to $4 \mathrm{KHz}$. The sound power level increased from $5 \mathrm{KHz}$ to $16 \mathrm{KHz}$ and it approached the plain nozzle condition at the highest frequency. With these tubes the overall sound power level was $116 \mathrm{db}$ which was $4.5 \mathrm{db}$ less than for the convergent nozzle. By adding the multiple shrouds to the tubes the sound power level was decreased over the frequency range of $100 \mathrm{~Hz}$ to 1 $\mathrm{KHz}$ from that of the multiple tube configuration. And over the frequency range of $1 \mathrm{KHz}$ to $6.5 \mathrm{KHz}$ the sound power level was slightly higher than for the multiple tubes. Over the frequency range of 6.5 to $16 \mathrm{KHz}$ the sound power level was nearly constant and lower than for the tubes. The overall sound power level was $112.7 \mathrm{db}$ which was $7.8 \mathrm{db}$ lower than observed for the convergent nozzle. Thus, the addition of the multiple shrouds to the tubes increased the suppression by $3.3 \mathrm{db}$, and similar results were observed in Ref. 7 and summarized in Ref. 10 . 


\section{0 CONCLUSIONS}

For subsonic jet Mach numbers, including the sonic Mach number, the velocity on the axis remained constant over a distance of approximately 5 diameters before decreasing in the fully developed subsonic turbulent region as $\mathrm{x}^{-1}$.

With a convergent nozzle at a jet Mach number of 1.4 , the sonic velocity on the axis occurred at 13.7 diameters from the nozzle exit and the length of the supersonic region was proportional to $M_{j}^{2}$. Downstream of the sonic point in the fully subsonic turbulent flow region the velocity decayed as $x^{-1}$, similar to that observed for subsonic jets.

Piezoelectric impact pressure fluctuations were determined on the jet axis, and for subsonic jet Mach numbers of 0.6 to 1.0 the peak pressure fluctuations occurred at approximately 9 diameters from the nozzle exit. For supersonic Mach number of 1.4 the peak pressure fluctuation occurred at 12.5 diameters, just ahead of the sonic point. In the fully developed subsonic turbulent jet the impact pressure fluctuations decreased as $\mathrm{x}^{-1.74}$ for both subsonic and supersonic jets:

The highest overall sound pressure levels occurred at an angular position of $19.1^{\circ}$ from the jet axis for jet Mach numbers of 0.6 to 1.4. For subsonic jets the sound pressure levels decreased monotonically with angular position from the jet axis. But for jet Mach numbers of 1.2 and 1.4 from a convergent nozzle, the overall sound pressure level decreased from $19.1^{\circ}$ to $43.8^{\circ}$ and then remained nearly constant for larger angular positions because of the shock bottles.

Power spectra for subsonic jets were quite similar with the peak power occurring at approximately $4 \mathrm{KHz}$ for Mach numbers of 0.60 to 1.0. At a jet Mach number of 1.4 the peak power occurred at a frequency of $5 \mathrm{KHz}$ which corresponds to a strouhal number of 0.64 .

Near-field sound pressure levels were determined with microphones placed radially at 2 to 8 diameters away from the nozzle exit. The overall sound pressures increased with axial distance and the variations were quite similar for jet Mach numbers of 0.6 to 1.0 . But for Mach numbers of 1.2 and 1.4 the sound pressure levels at the jet exit plane were much greater than observed for the sonic jet and the variations of the sound pressure level with distance were quite different than those observed for subsonic jets.

From the near-field pressure measurements the distributions of the acoustic power transmission through a cylindrical surface for a given radial location of the microphone were determined for Mach 
numbers of 0.6 to 1.4 , and were found to be quite different for subsonic and supersonic jets. For Mach numbers of 1.2 and 1.4 the acoustic power distribution increased almost linearly from the jet exit to the sonic location. And by assuming acoustic power decay as $x^{-6}$ in the subsonic region, the overall sound power levels were determined and the values agreed closely with those observed in the far-field.

Overall sound power levels for Mach numbers of 0.6 to 1.5 were determined and compared with the subsonic theory of Iighthill and supersonic theory of Nagamatsu and Horvay. At a jet Mach number of 0.6 the overall sound power level agreed with Lighthill's prediction, but at higher Mach numbers the experimental power levels were higher than the prediction.

At supersonic jet Mach numbers the aerodynamic flow model and the acoustic power distribution agreed with the assumption used in the derivation of the supersonic jet noise theory of Nagamatsu and Horvay. The exponents $\alpha$ and $\beta$ in the theory were evaluated for convergent and parallel flow nozzles as functions of the jet Mach number. Overall sound power levels for jet Mach numbers of 0.6 to 1.4 were compared with the supersonic theory.

The velocity contours for subsonic jet Mach numbers of 0.6 to 1.0 were quite similar with the core extending to approximately 4 diameters on the axis for the 1 in. convergent nozzle. At supersonic Mach numbers of 1.2 to 1.4 the distance from the nozzle exit to the sonic location on the axis increased as $\mathrm{M}_{j}^{2}$, and the subsonic mixing region increased very rapidly with distance.

Contours of the piezoelectric impact pressure fluctuations for Mach numbers of 0.6 to 1.0 were similar with the peak occurring in a toroidal region located approximately 4 to 6 diameters from the nozzle. For supersonic Mach numbers of 1.2 and 1.4 the peak fluctuations occurred close to the sonic velocity contour in the supersonic region of the jet.

Analyses were made to show that the outputs of the piezoelectric impact and static pressure probes were related to the local mean velocity and density and turbulent velocity fluctuations. The turbulent velocity fluctuations in subsonic jets determined with hot-wire and laser doppler velocimeter technique were correlated with the piezoelectric impact and static pressure probe data and good agreement was obtained.

The peak static pressure fluctuations occurred at approximately 10 diameters for subsonic jet Mach numbers of 0.6 to 1.0 , and downstream of the peak location the rms static pressure fluctuation decreased approximately as $x^{-1.95}$. For supersonic Mach numbers of 
1.2 and 1.4 the peak static pressure fluctuations occurred just ahead of the sonic location on the axis.

Contours of constant rms piezoelectric static pressure fluctuations for jet Mach numbers of 0.6 to 1.0 were similar with the peak occurring close to $r / r_{0}=1.0$ for axial locations near the nozzle exit. At distances farther downstream the peak location moved farther out radially. For Mach numbers of 1.2 and 1.4 the contours of the constant static pressure fluctuations were quite different than the contours for subsonic jet Mach numbers. The peak static pressure fluctuations occurred close to the jet axis ahead of the sonic location for these Mach numbers.

A supersonic jet noise suppressor consisting of 191 tubes and shrouds was investigated at jet Mach numbers of 1.4 and 0.7 , and this suppressor decreased the primary jet Mach number drastically for both jet Mach numbers. Axial surveys with piezoelectric impact and static pressure probes indicated large reductions in the impact and static pressure fluctuations from the values existing for the convergent nozzle at supersonic and subsonic Mach numbers.

With multiple tubes the Mach 1.4 jet noise level was reduced 15.3 $\mathrm{db}$ and for the Mach 0.7 jet the reduction was $4.5 \mathrm{db}$. By adding the multiple shrouds to the tubes the noise level reduction for the Mach 1.4 jet was increased to $20.5 \mathrm{db}$, while the reduction for the Mach 0.7 jet was $7.8 \mathrm{db}$.

A supersonic jet noise suppressor consisting of a single shroud, six small rods, and induced flow indicated the large reduction in the jet velocity at the exit of the shroud with and without the induced flow. The Mach 1.4 jet noise level was reduced $14.2 \mathrm{db}$ with the shroud, rods, and induced flow, and without the induced flow the reduction was only $4.5 \mathrm{db}$ because of the shock waves in the shroud.

Sound pressure level spectra were determined for eight angular positions for the shroud with and without induced flow. The results indicated that the shielding of acoustic radiation from the supersonic region by the shroud and induced flow was effective, but the shock wave generated noise within the shroud for no induced flow propagated to the outside through subsonic flow with little attenuation. 


\section{REFERENCES}

1. Nagamatsu, H.T., Sheer, R.E.; Jr.; and Wells, R.J., "Supersonic Jet Exhaust Noise Reduction with Rods, Shroud, and Induced Flow," Proc. AFOSR-UTIAS Symposium on Aerodynamic Noise, Toronto, May 1968 .

2. Nagamatsu, H.T., Pettit, W.T., and Sheer, R.E., Jr., "Flow and Acoustic Measurements on a Convergent Nozzle Supersonic Jet Ejector," General Electric Research and Development Center, Report No. 69-C-156, April 1969.

3. Nagamatsu, H.T. and Horvay, G., "Supersonic Jet Noise," AIAA Paper No. 70-237 (1970).

4. Nagamatsu, H.T., Sheer, R.E., Jr., and Gill, M.S., "Flow and Acoustic Characteristics of Subsonic and Supersonic Jets From Convergent Nozzle," AIAA Paper No. 70-802 (1969).

5. Nagamatsu, H.T., Sheer, R.E., Jr., and Horvay, G., "Supersonic Jet Noise Theory and Experiments," Basic Aerodynamic Noise Research Conf., NASA SP-207 (1969).

6. Nagamatsu, H.T., Sheer, R.E., Jr., and Bigelow, E.C., "Mean and Fluctuating Velocity Contours and Acoustic Characteristics of Subsonic and Supersonic Jets," AIAA Paper No. 72-157 (1972).

7. Nagamatsu, H.T., Sheer, R.E., Jr., and Gill, M.S., "Flow and Acoustic Characteristics of 191 Tubes and 191 Shrouds Supersonic Jet Noise Suppressor," AIAA Paper No. 71-153 (1971).

8. Nagamatsu, H.T. and Sheer, R.E., Jr., "Flow, Thrust, and Acoustic Characteristics of 50 Tubes with 50 Shrouds Supersonic Jet Noise Suppressor," AIAA Paper No. 72-642 (1972).

9. Nagamatsu, H.T., Sheer, R.E., Jr., and Bigelow, E.C., "Subsonic and Supersonic Jet Flow and Acoustic Characteristics and Supersonic Suppressors," General Electric Research and Development Center, Report No. 72CRD264 (1972).

10. Nagamatsu, H.T. and Sheer, R.E., Jr., "Supersonic Jet Noise Suppressor Results and Prediction Methods," General Electric Research and Development Center, Report (In Process).

11. Lassiter, L.W. and Hubbard, H.H., "The Near Field Noise of Static Jets and Some Model Studies of Devices for Noise Reduction," NACA Rept. 1261 (1954).

12. Gerrard, J.H.,..."An Investigation of the Noise Produced by Subsonic Air Jet," J. Aero. Sci., Vol. 23, 855-866 (1956). 
13. Mollo-Christensen, E. and Narasimha, R., "Sound Emission from Jets at High Subsonic Velocity," J. Fluid Mech., Vol. 8, 4960 (1960).

14. Laurence, S.C., "Intensity, Scale, and Spectra of Turbulence in Mixing Region of Free Subsonic Jet," NACA, Rept. 1292 (1956).

15. Davies, P.O.A.L., Ko, N.W.M., and Bose, B., "The Local Pressure Field of Turbulent Jets," ARC CP 989 (1967).

16. Potter, R.C. and Jones, J.H., "An Experiment to Locate the Acoustic Sources in a High Speed Jet Exhaust Stream," Wyle Laboratories Report (1967).

17. Lighthill, M.J.; "Jet Noise," AIAA Journal, Vol. 1, No. 7, 1507-1517 (1963).

18. Dosanjh, D.S. and Montegani, F.J., "Underexpanded Jet Noise Reduction Using Radial Flow Impingement," AIAA J., Vol. 7, No. 3, p. 458-464 (1969).

19. Love, E.S., Grigsby, C.E., Lee, L.P., and Woodling, M., "Experimental and Theoretical Studies of Axisymmetric Free Jets," J.A. Sci., 25, p. 791-799 (1958).

20. Warren, W.R., "An Analytical and Experimental Study of Compressible Free Jets," Doctoral Dissertation Princeton Univ. (1957).

21. Eggers, S.M., "Velocity Profiles and Eddy Viscosity Distribution Downstream of a Mach 2.22 Nozzle Exhausting to Quiescent Air," NASA TN D-3601 (1966).

22. Ribner, H.S.; "The Generation of Sound by Turbulent Jets," Adv. Appl. Meçh., Vol. 8, Academic Press, Inc., N.Y. (1964).

23. Kraichnan, R.H.; "Pressure Field within Homogeneous Anisotropic Turbulence," JASA, Vol. 28, 64-72 (1956).

24. Phillips, O.M., "On the Generation of Sound by Supersonic Turbulent Shear Layers," J. Fluid Mech., Vol. 9, Part I, $1-28$ (1960).

25. Williams, J.E.F., "The Noise from Turbulence Convected at High Speed," Phil. Trans. Roy. Soc. London, Series A225, 479-503 (1963). 
26. Ollerhead, J.B., "On the Prediction of the Near Field Noise of Supersonic Jets," Wyle Laboratories, NASA CR-857 (1967).

27. Benzakein, M.J. and Knott, P.R., "Supersonic Jet Exhaust Noise," USAF Aero Propulsion Laboratory, Report No. AFAPL-TR-72-52 (1972).

28. Laurence, J.C., "Intensity, Scale, and Spectra of Turbulence in Mixing Region of Free Subsonic Jet," NACA Report 1292 (1956).

29. Mayes, W.H., Lanford, W.E., and Hubbard, H.H., "Near Field and Far Field Noise Surveys of Solid Fuel Rocket Engines for a Range of Nozzle Exit Pressures," NASA TN D-2l (1959).

30. Davies, P.O.A.I., Fisher, M.J., and Barratt, M.J., "The Characteristics of the Turbine in the Mixing Region of a Round Jet," J. Fluid Mech., Vol. 15, Part 2, 337-366 (1963).

31. Arndt, R.E., Tran, N., and Barefoot, G., "Turbulence and Acoustic Characteristics of Screen Perturbed Jets," AIAA Paper No. 72-644 (1972).

32. Siddon, T.E., "On the Response of Pressure Measuring Instrumentation in Unsteady Flow," Univ. of Toronto, UTIAS Rept. 136 (1969).

33. Nakamura, A., Sugiyama, A., Tanaka, T., and Matsumoto, M., "Experimental Investigation for Detection of Sound-Pressure Level by a Microphone in an Airstream," JASA, Vol. 50, No. 1, 40-46 (1971).

34. Fuchs, H.V., "Energy-Balance for Small Fluctuations in a Moving Medium," Univ. of Southampton, Inst. of Sound and Vib., Rept. 18 (1969).

35. Schairer, G.S., O'Keefe, J.V., and Johnson, P.E., "Perspective of SST Aircraft Noise Problem," AIAA Paper No. 68-1023 (1968).

36. Stringas, E.J.,. "Review of Current Multi-Element Aerodynamic Characteristics," General Electric Flight Propulsion Div. Report TN-70007 (1970).

37. Harris, V.E., "Model/Ful1 Scale GE4 Block I Jet Noise Testing," General Electric Flight Propulsion Div. Report TM No. 68-15 (1968).

38. Pettit, W.T., III., "The Laser Doppler Velocimeter for Measuring Turbulence in Gas Flows," Polytechnic Inst. of Brooklyn, M.S. Thesis (1970). 
39. Nagamatsu, H.T., Geiger, R.E., snd Sheer, R.E., Jr., "Hypersonic Shock Tunnel," Jour. ARS, Vol. 29, 332-340 (1959).

40. Mirels, H., "Laminar Boundary Layer. Behind Shock Advancing Into Stationary Fluid," NACA TN 3401 (1955).

41. Jones, I.S.F., "Fluctuating Turbulent Stresses in the Noise-Producing Region of a Jet," J. Fluid Mech., 36, Part 3, 529-543 (1969).

42. Pao, S.P., "A Generalized Theory on the Noise Generation from Supersonic Shear Layer," Jour. Sound and Vibration, 19, 401410 (1971).

43. Chen, C.Y. and Knott, P.R., "Analytical Models for Near-Field Jet Noise Calculation," General Electric Aircraft Engine Group, Report TM 72-349 (1972).

44. Tatge, R.B. and Wells, R:J., "Model Jet Noise Study at Alplaus Facility," General Electric Engineering Laboratory, Rept. 61GL25 (1961).

45. Schlichting, H., Boundary Layer Theory, McGraw-Hill Book Co., N.Y. (1960). 
ACOUSTIC POWER LEVELS FOR CONVERGENT NOZZLE, 191 TUBES, 191 SHROUDS, AND SINGLE SHROUDS

A. Outside Air Temperature $\sim 20^{\circ} \mathrm{F}$

1) Pressure Ratio $=3.2, \mathrm{M}_{j}=1.4$

\section{Configuration}

1-9/16" Dia. Convergent Nozzle

191 Tubes

191 Tubes, 6" Long Shrouds $\left(\mathrm{x}_{1}=3 / 16^{\prime \prime}\right)$

191 Tubes, 6" Long Shrouds $\left(\mathrm{x}_{1}=1 "\right)$

191 Tubes, 6" Long Shrouds $\left(x_{1}=\right.$ $2 "$, no induced flow)

191 Tubes, $S_{12}^{\prime}$ Shroud

2) Pressure Ratio $=1.4, \mathrm{M}_{j}=0.71$

1-9/16" Dia. Convergent Nozzle

191 Tubes, 6" Long Shrouds

$\left(x_{1}=3 / 16^{\prime \prime}\right)$

B. Outside Air Temperature $\sim 68^{\circ} \mathrm{F}$

1) Pressure Ratio $=3.2, \mathrm{M}_{j}=1.4$

1-9/16" Dia. Convergent Nozzle

191 Tubes

191 Tubes, 6" Long Shrouds

$\left(x_{1}=3 / 16^{\prime \prime}\right)$

191 Tubes, 3.335" Long Shrouds $\left(x_{1}=3 / 16^{\prime \prime}\right)$

$\mathrm{L}_{\mathrm{W}}$

\section{(db re $10^{-13}$ watts)}

154.3

138.3

132.4

136.7

$135: 5$

138.7

123.6

114.2

150.7

135.5

130.3

131.7
Suppression (ab from plain jet)

$-16.0$

$-21.9$

$-17.6$

$-18.8$

$-15.6$

$-9.4$

0

$-15.2$

$-20.4$

$-19.0$ 


\section{TABLE I (Continued)}

191 Tubes, 1.667" Long Shrouds $\left(\mathrm{x}_{1}=3 / 16^{\prime \prime}\right)$

191 Tubes, $S_{3.8}$ Shroud
135.2

137.0
$-15.5$

$-13.7$ 


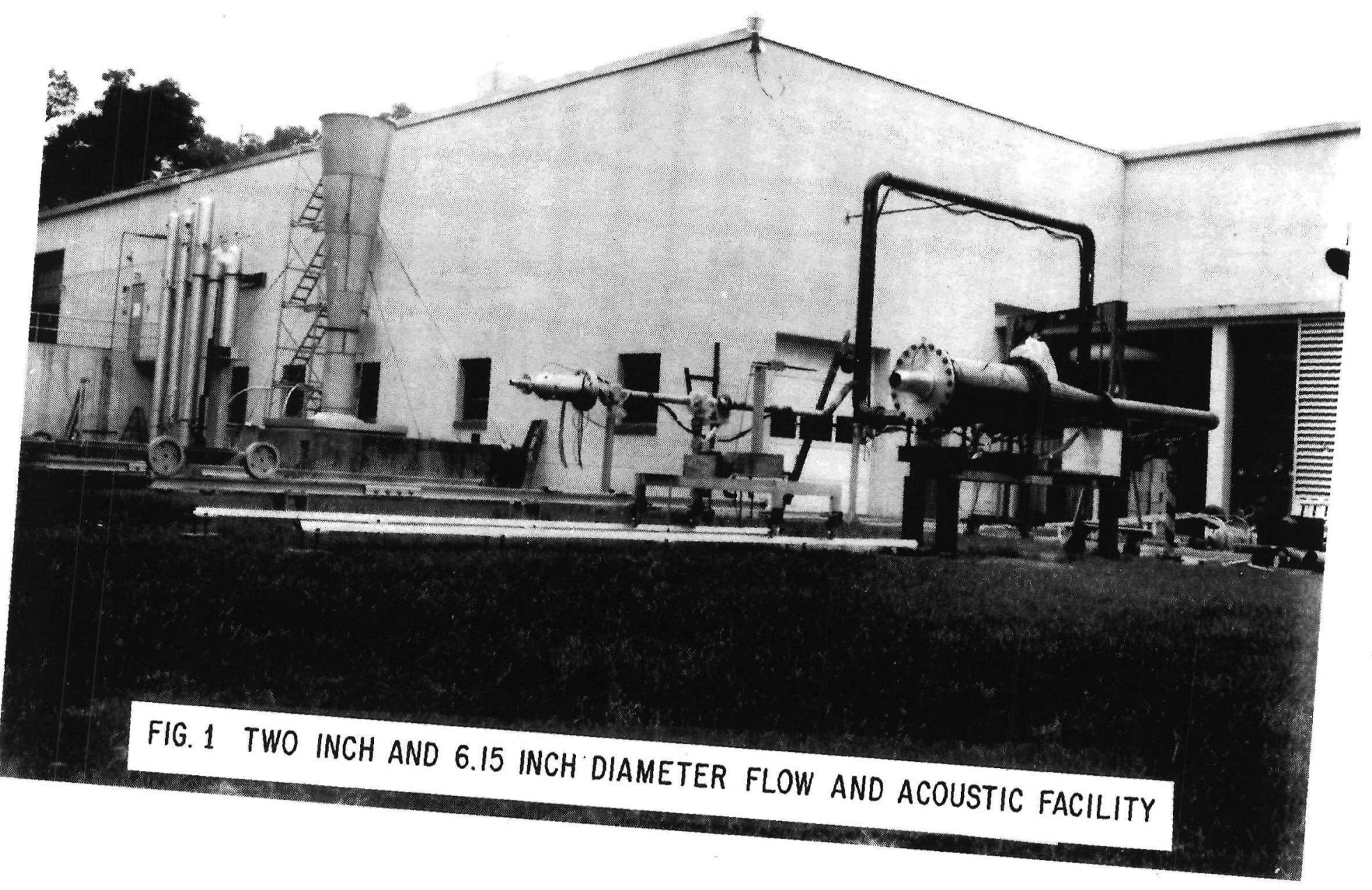




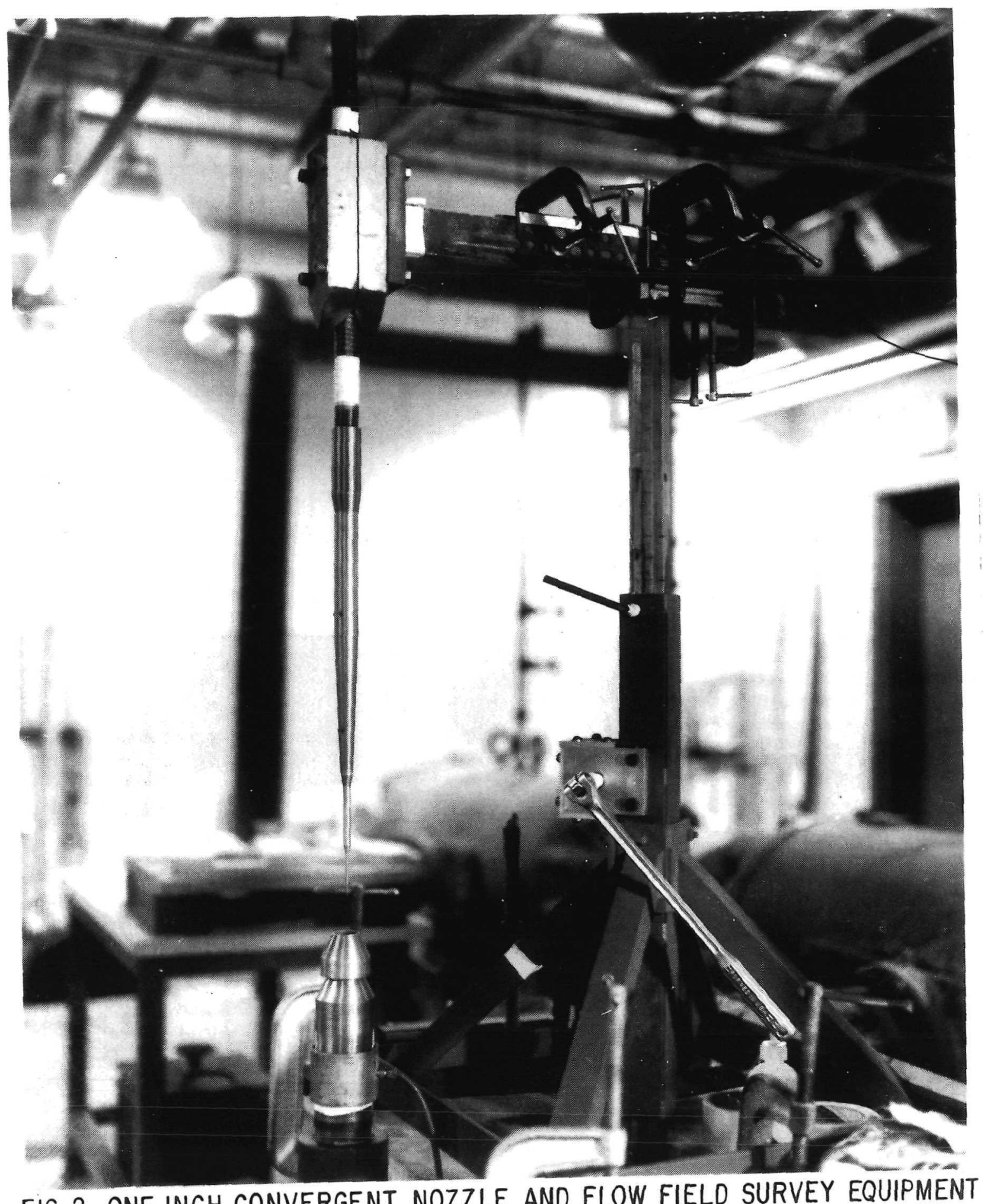

FIG. 2 ONE INCH CONVERGENT NOZZLE AND FLOW FIELD SURVEY EQUIPMENT 


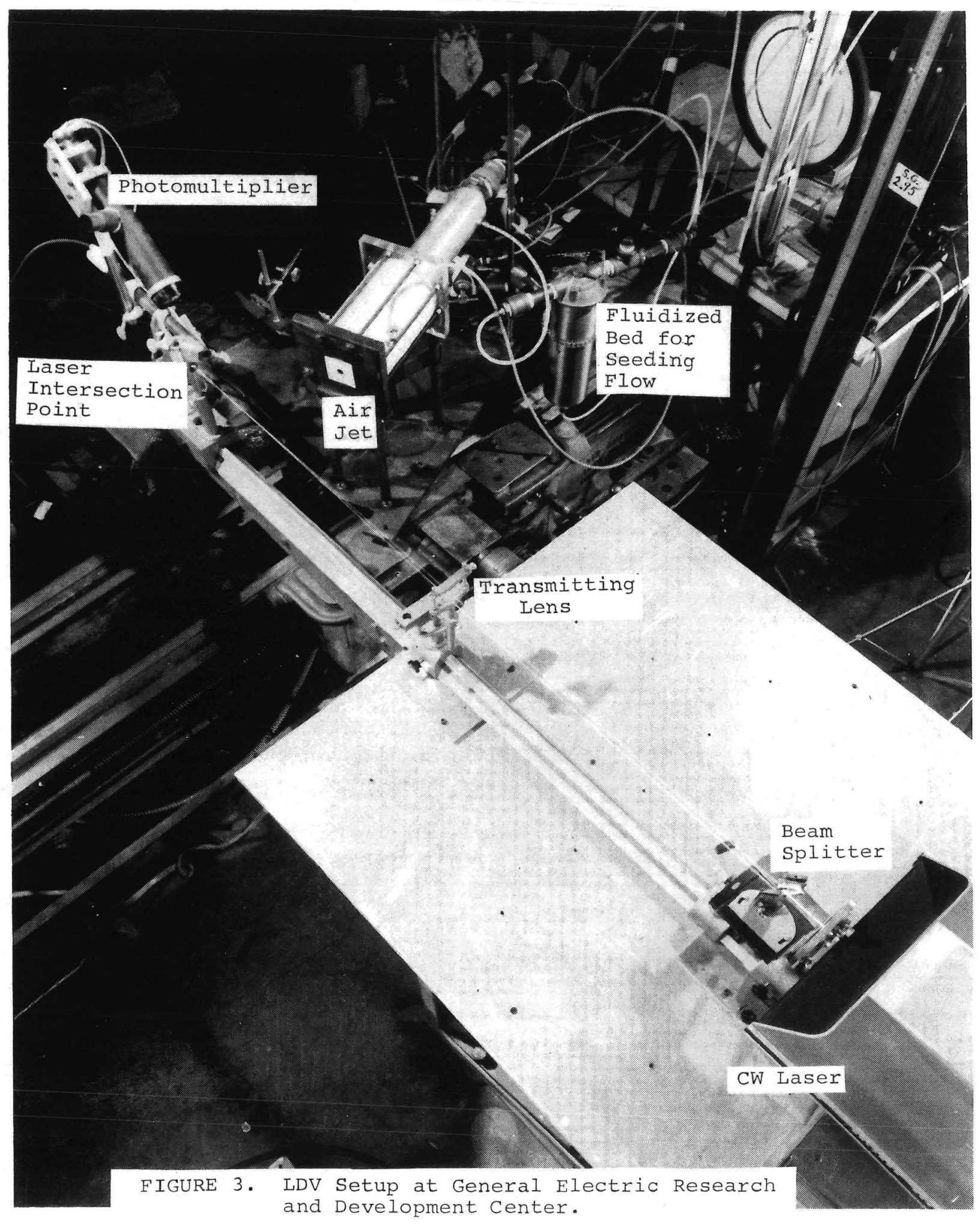




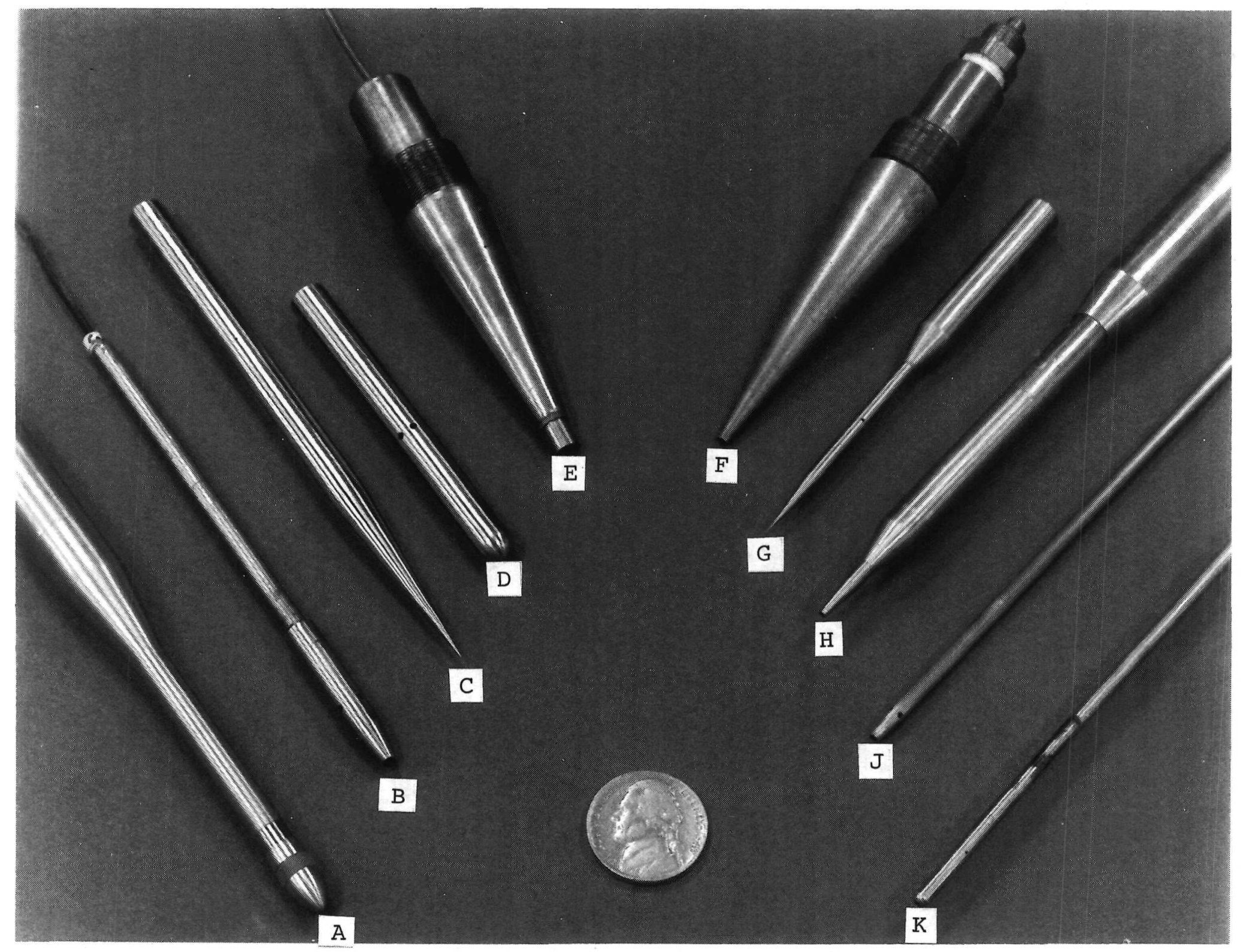

FIGURE 4. Probes for Jet Exhaust Surveys. 


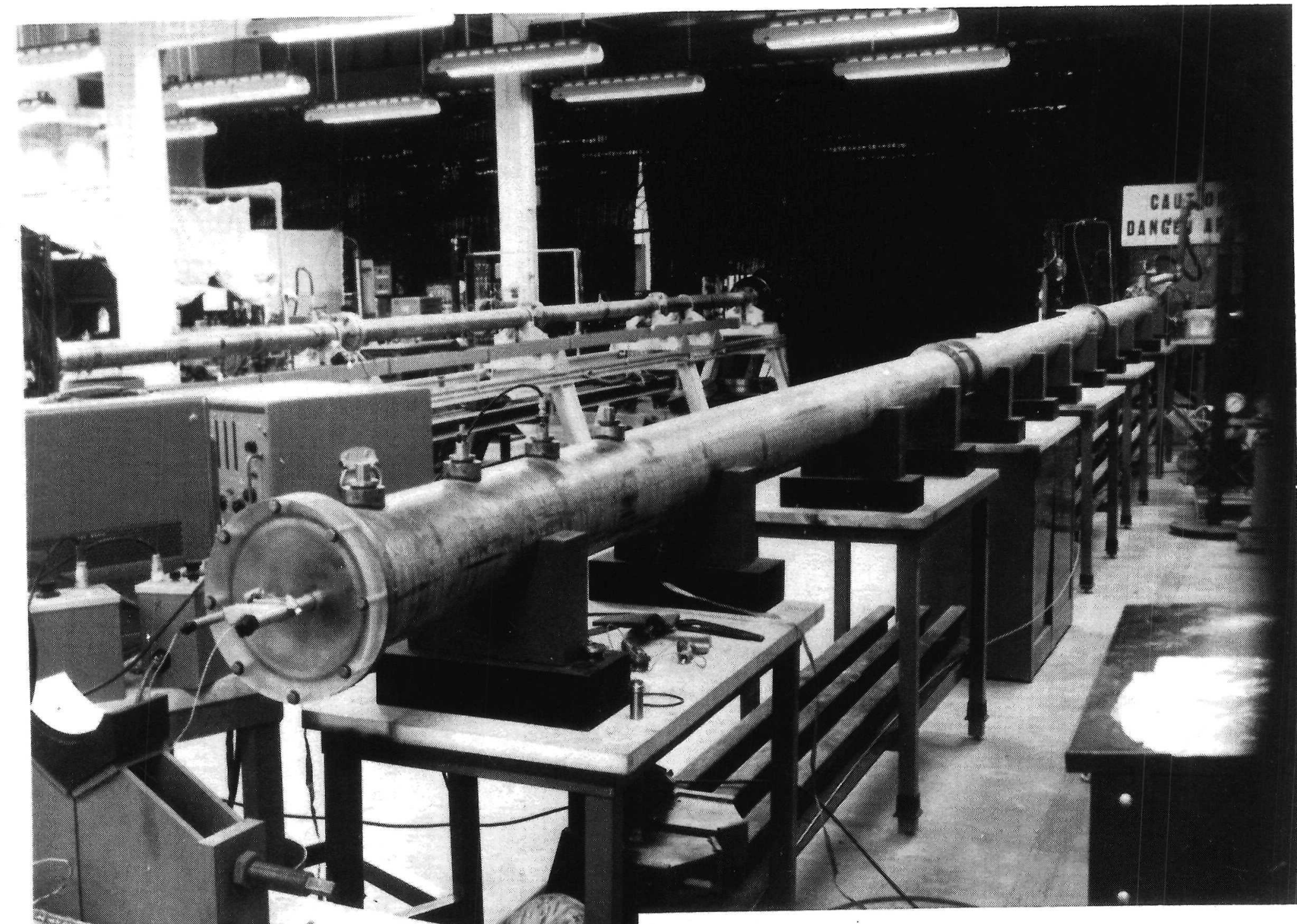

FIG. 5 EIGHT INCH CALIBRATION SHOCK TUBE 


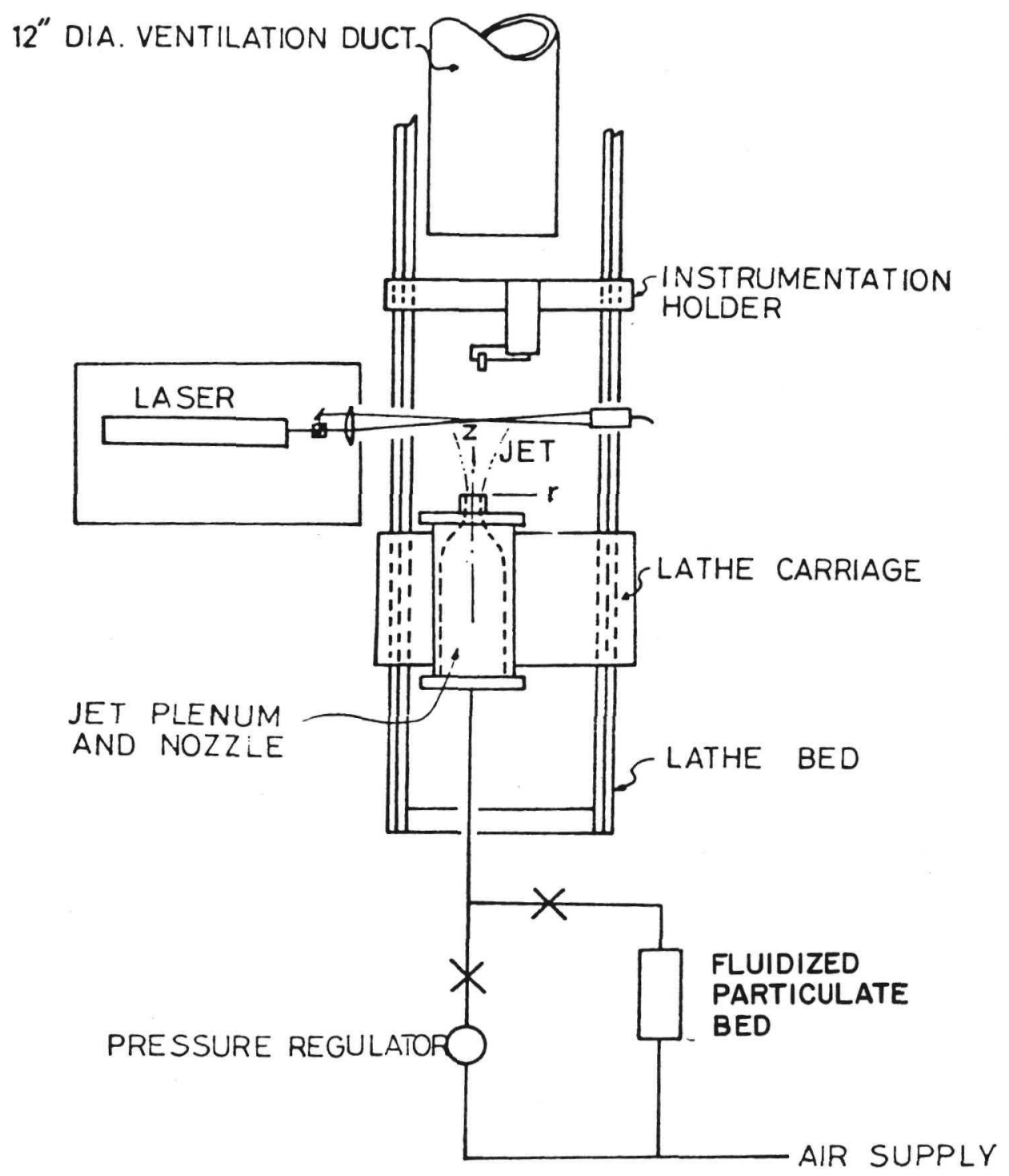

FIG. 6 EXPERIMENTAL ARRANGEMENT "FOR JET TURBULENCE MEASUREMENT WITH LASER DOPPLER VELOCIMETER 


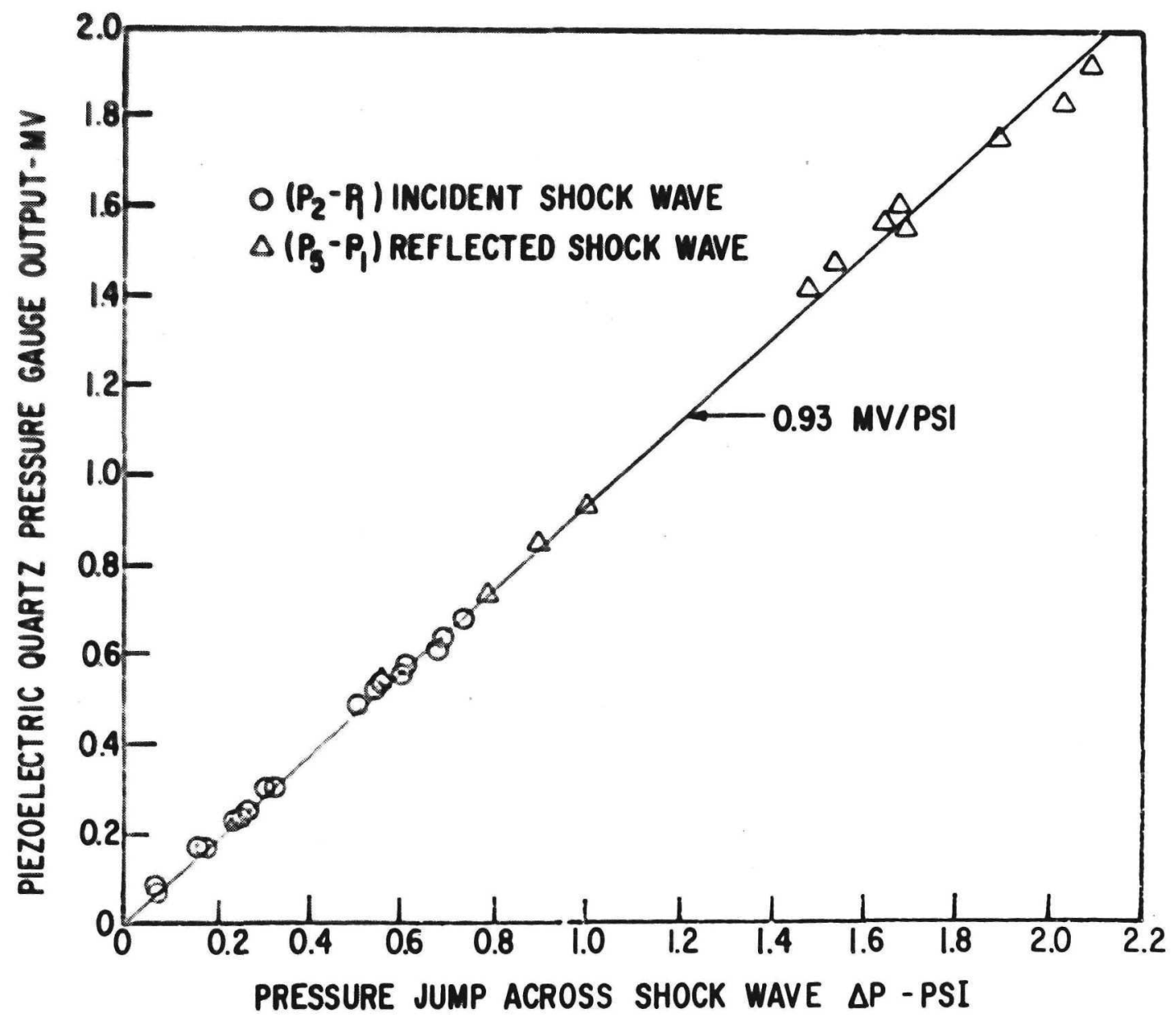

FIG. 7 PIEZOELECTRIC QUARTZ PRESSURE GAUGE OUTPUT AS A FUNCTION OF PRESSURE JUMP ACROSS SHOCK WAVES 


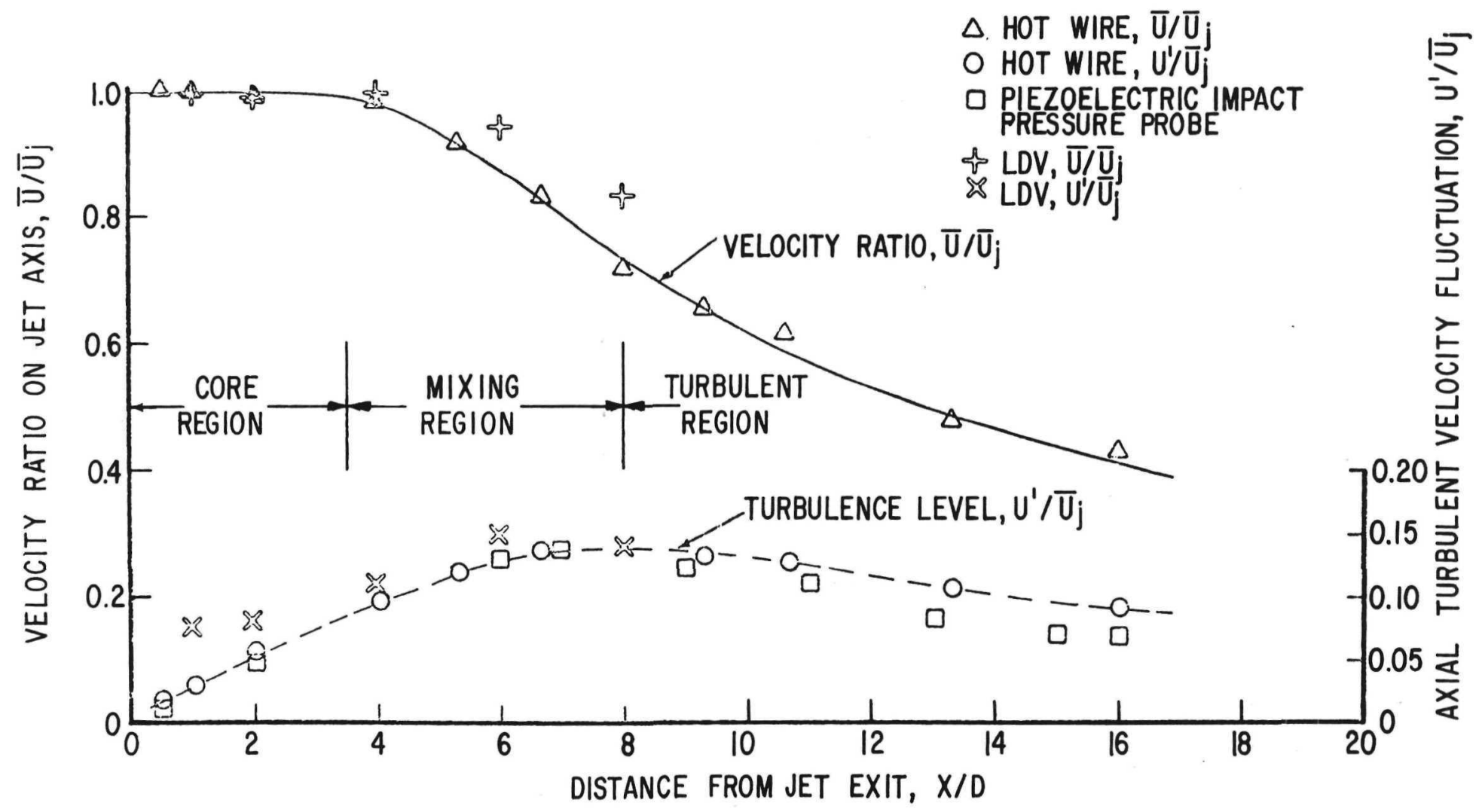

FIG.80 VELOCITY RATIO ON AXIS AND AXIAL TURBULENCE LEVEL WITH HOT-WIRE, LDV, AND PIEZOELECTRIC IMPACT PRESSURE PROBE FOR $3 / 4$ INCH

NOZZLE, $M_{j}=0.3$ 


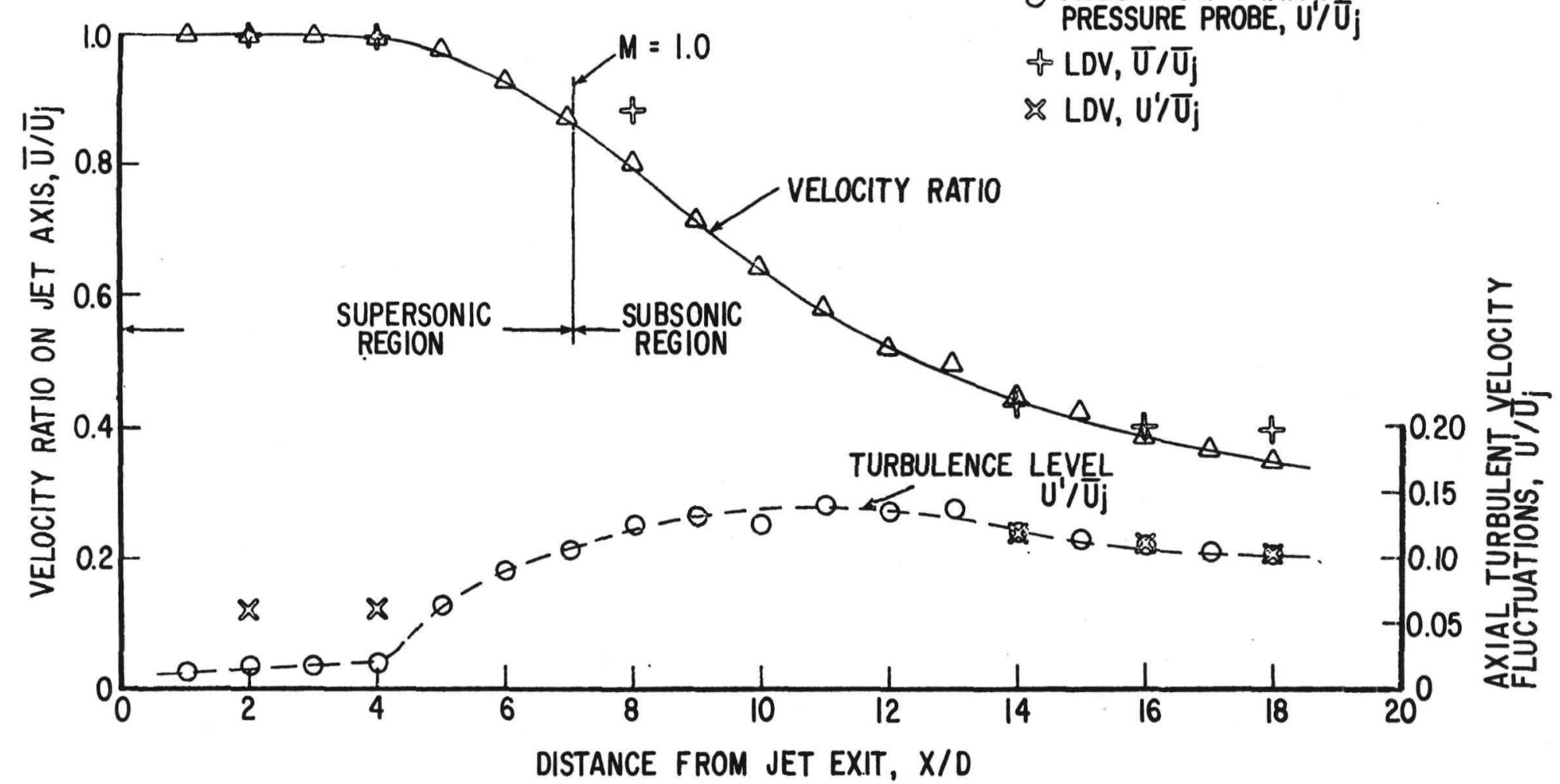

FIG. 8b VELOCITY RATIO ON AXIS AND AXIAL TURBULENCE LEVEL WITH PIEZOELECTRIC IMPACT PRESSURE PROBE AND LDV FOR $1 / 2$ INCH NOZZLE, $M_{j}=1.2$ 


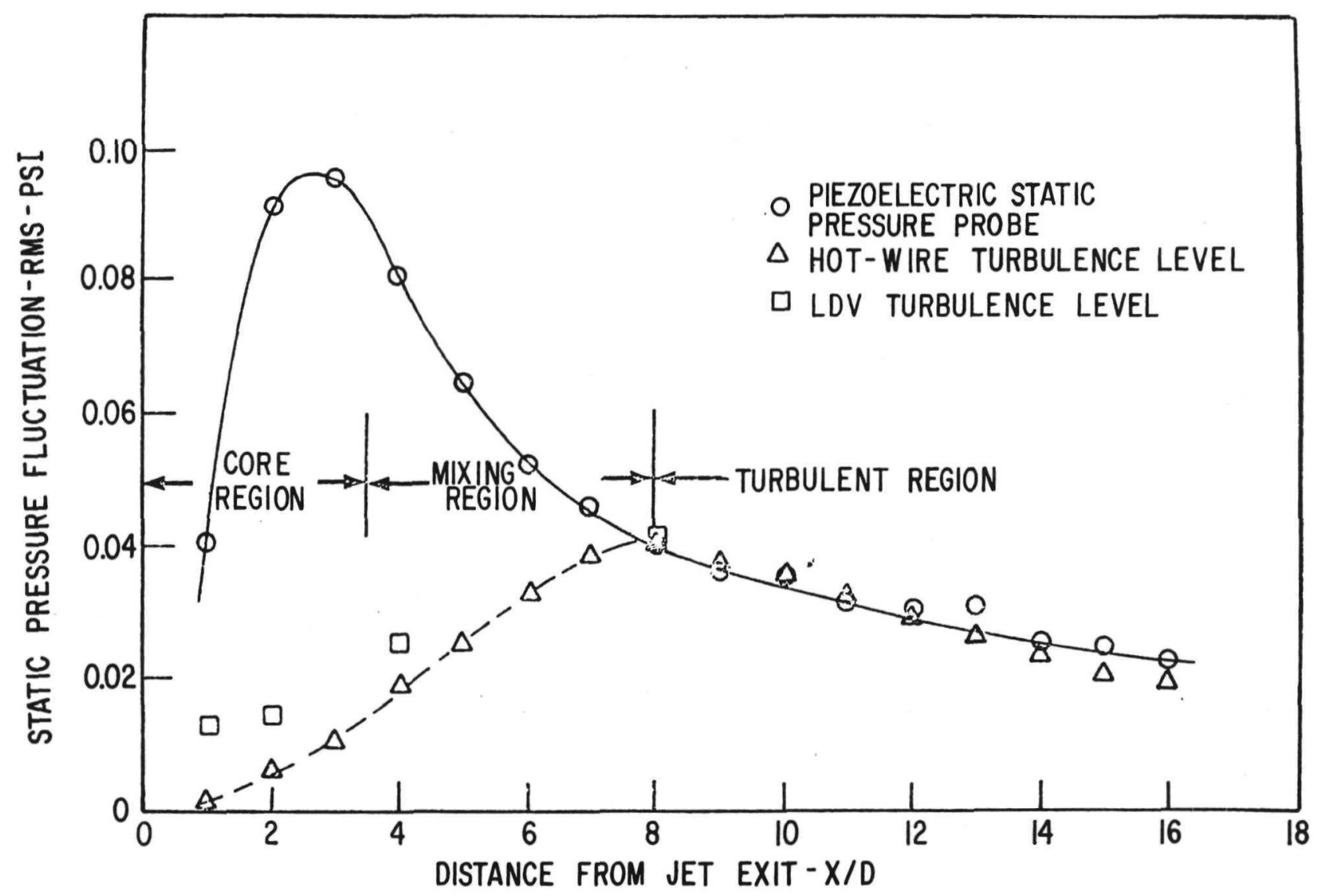

FIG. 9a AXIAL IRMS STATIC PRESSURE FLUCTUATIONS DETERMINED WITH PIEZOELECTRIC STATIC PRESSURE PROBE AND FROM HOT-WIRE TURBULENCE MEASUREMENTS FOR $3 / 4 \mathrm{INCH}$ NOZZLE - $M_{j}=0.3$ 


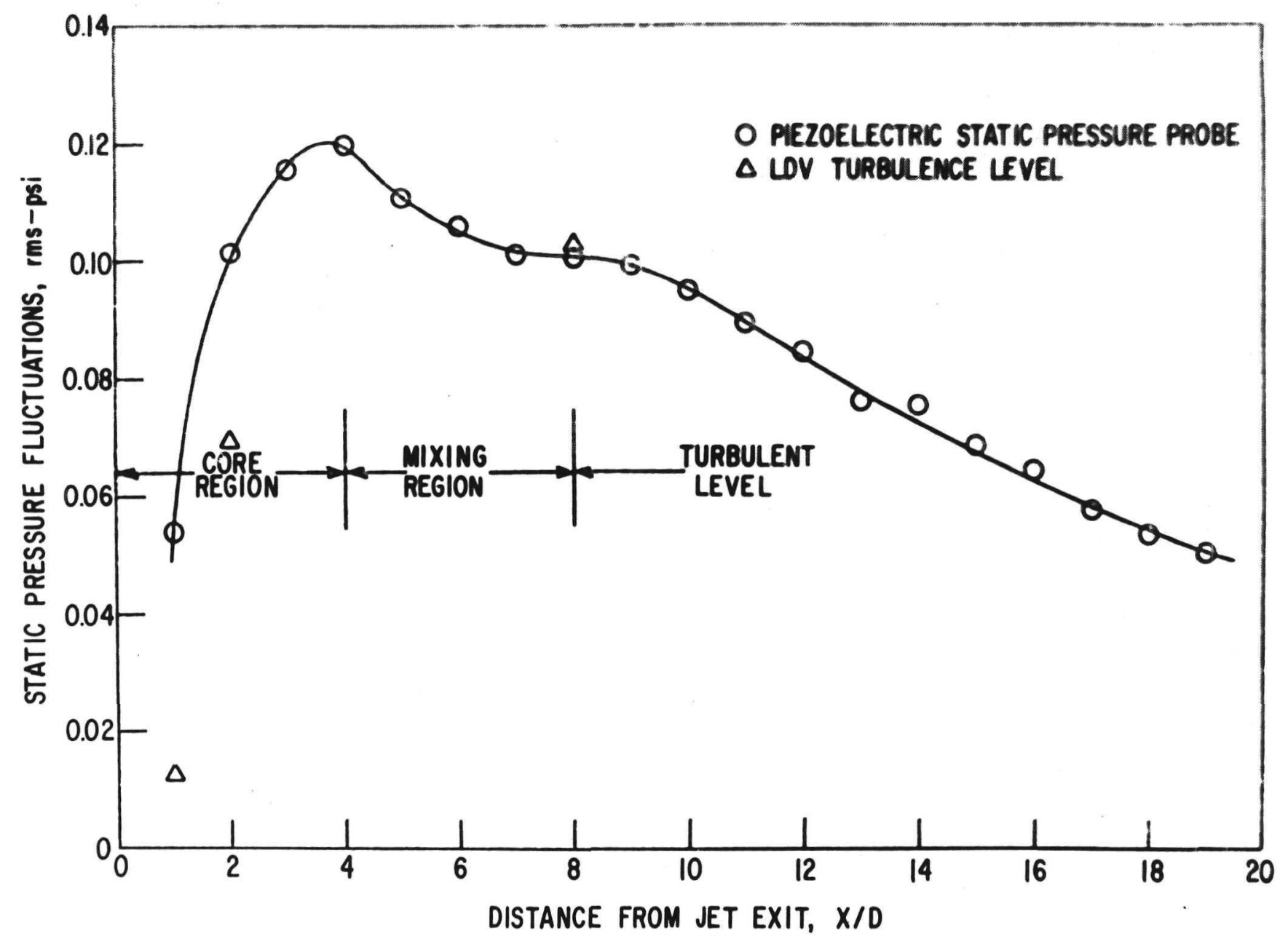

FIG. 9b AXIAL RMS STATIC PRESSURE FLUCTUATIONS DETERMINED WITH PIEZOELECTRIC STATIC PRESSURE PROBE AND LDV TURBULENCE MEASUREMENTS FOR 3/4 INCH NOZZLE $M_{j}=0.6$ 


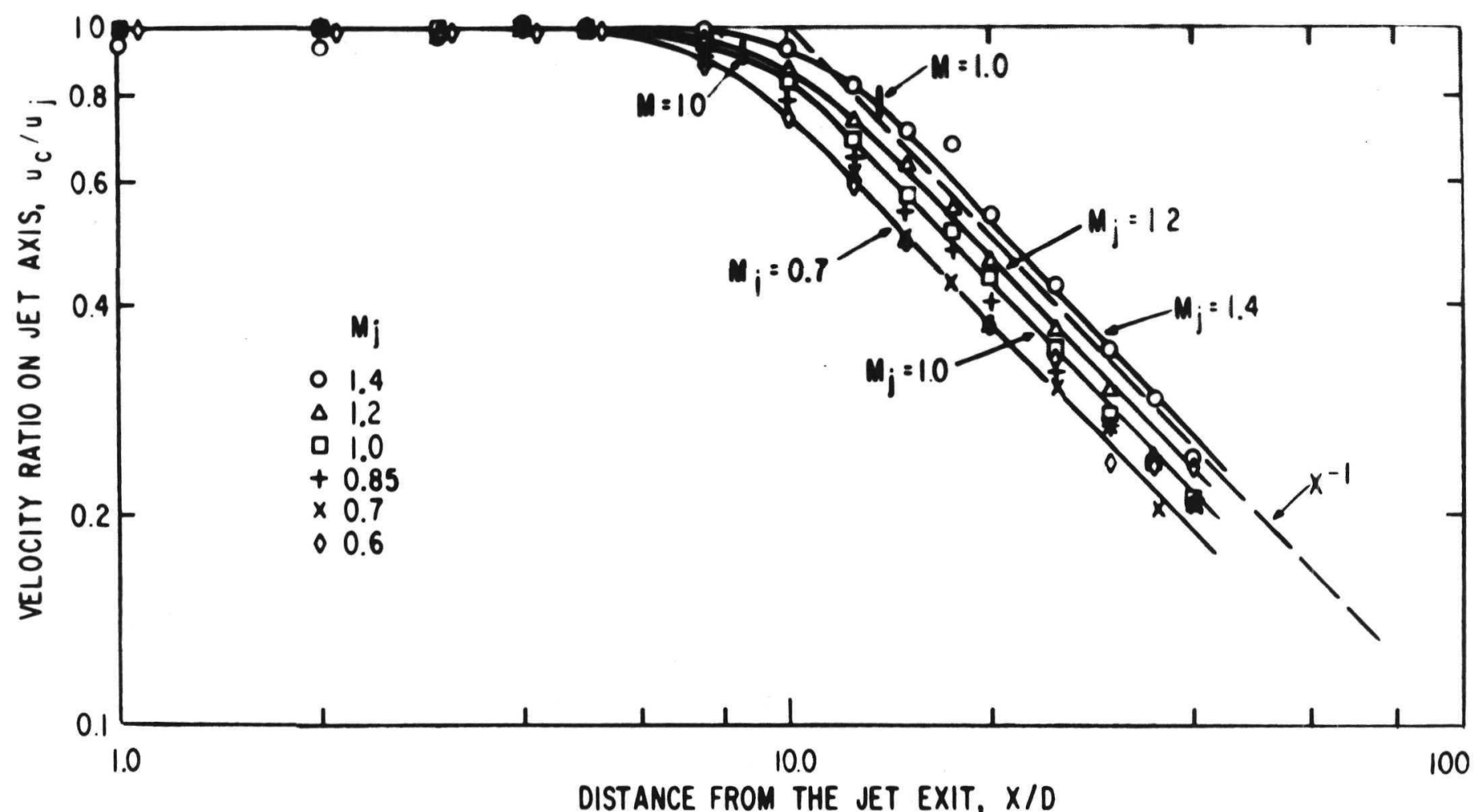

FIG.IOO VELOCITY RATIO ON JET AXIS FOR TWO INCH DIAMETER CONVERGENT NOZZLE. 


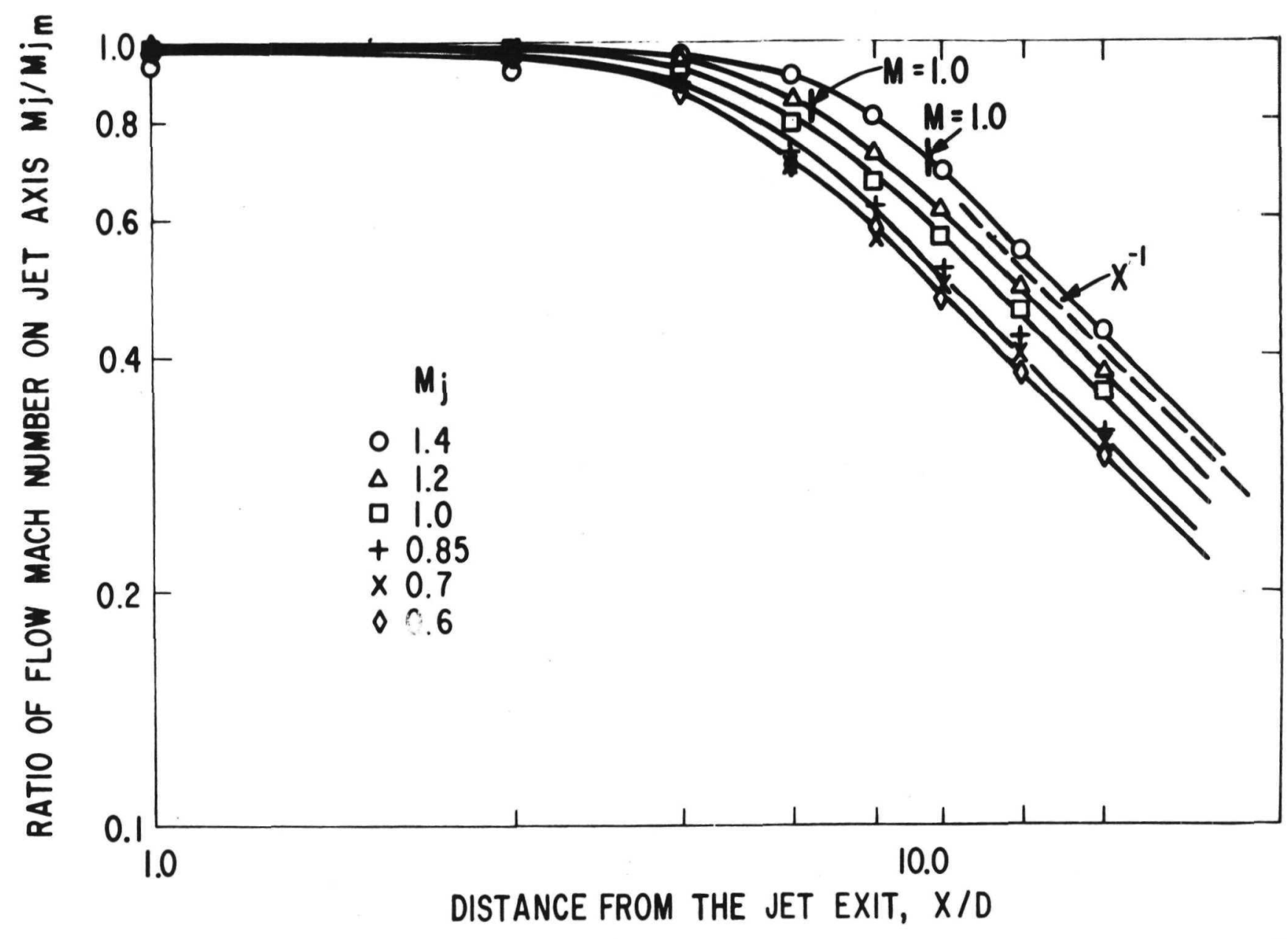

FIG.IOb FLOW MACH NUMBER RATIO ON JET AXIS FOR ONE INCH DIAMETER CONVERGENT NOZZLE. 
37ZZON INJ9YZANOJ Y YIJWVIO

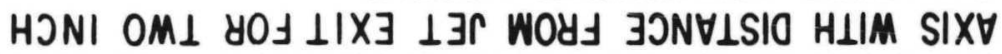

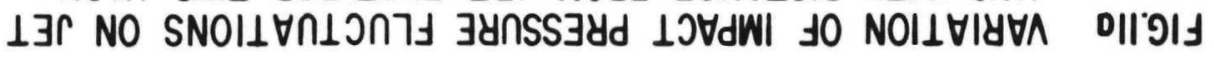

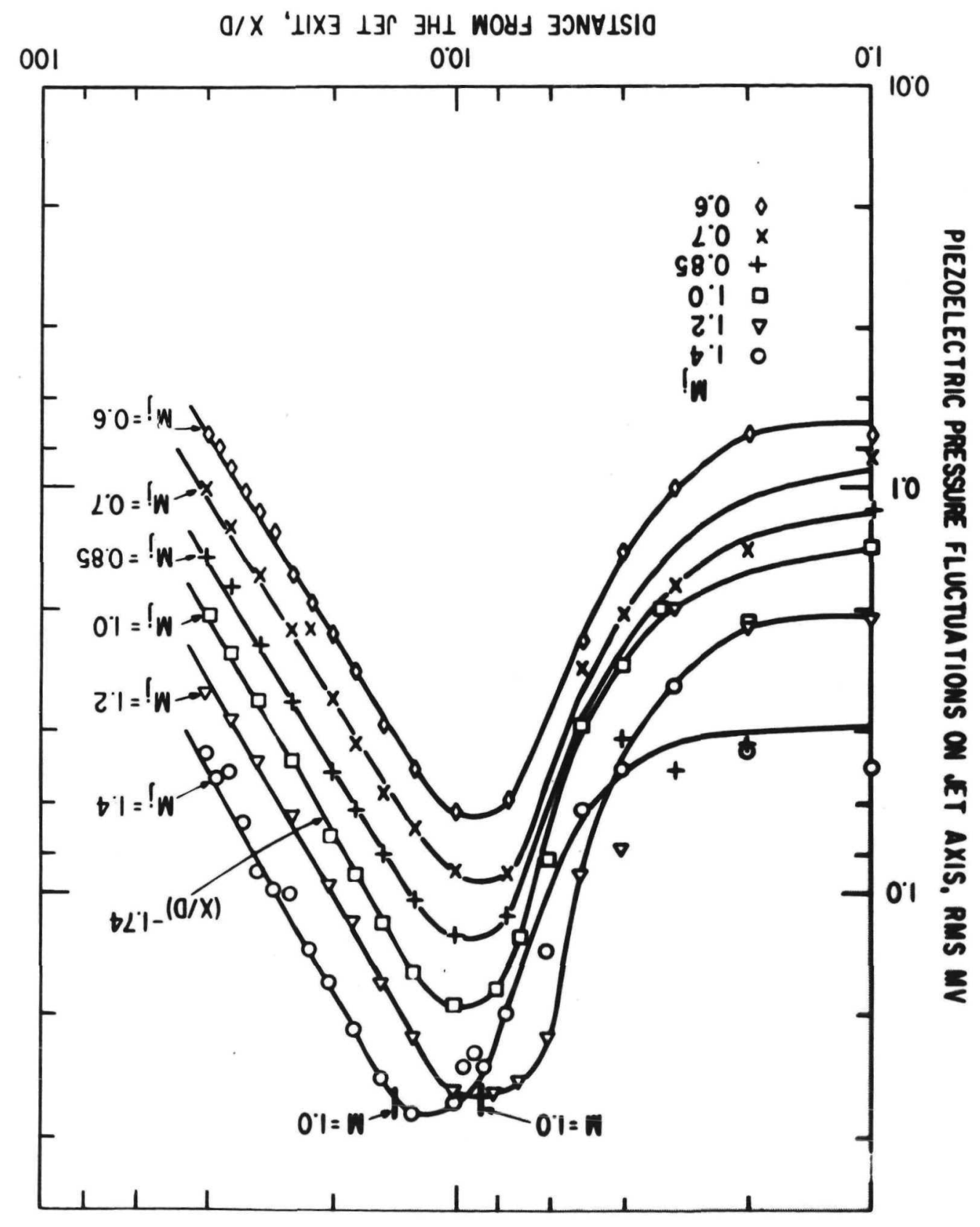




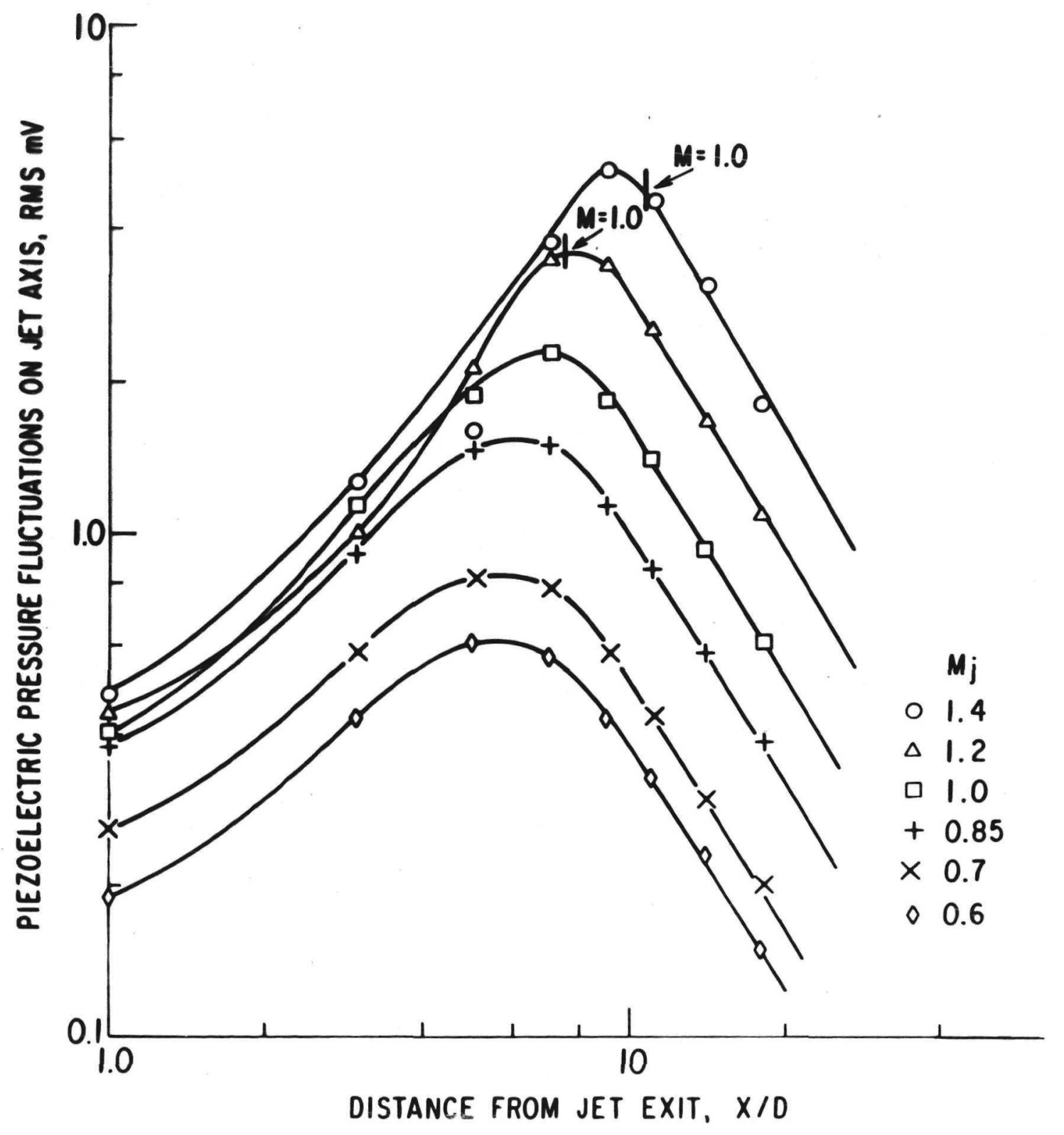

FIG.IIb VARIATION OF PIEZOELECTRIC PRESSURE FLUCTUATIONS ON JET AXIS WITH DISTANCE FROM JET EXIT FOR ONE INCH DIAMETER CONVERGENT NOZZLE. 

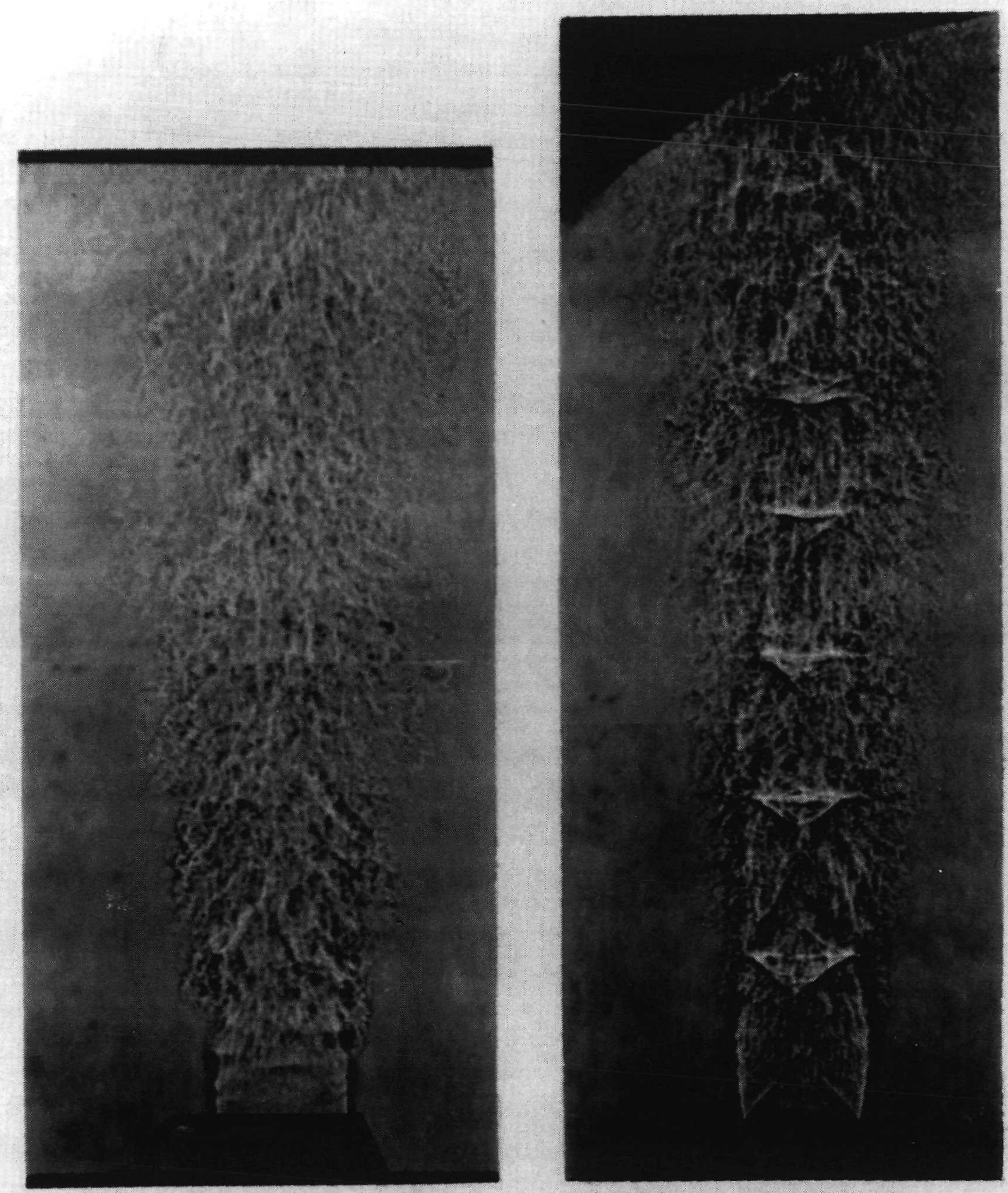

FIG.I2 SHADOWGRAPH PHOTOGRAPHS OF FLOW FROM A CONVERGENT NOZZLE AT MACH NUMBERS OF 1.0 AND 1.4 


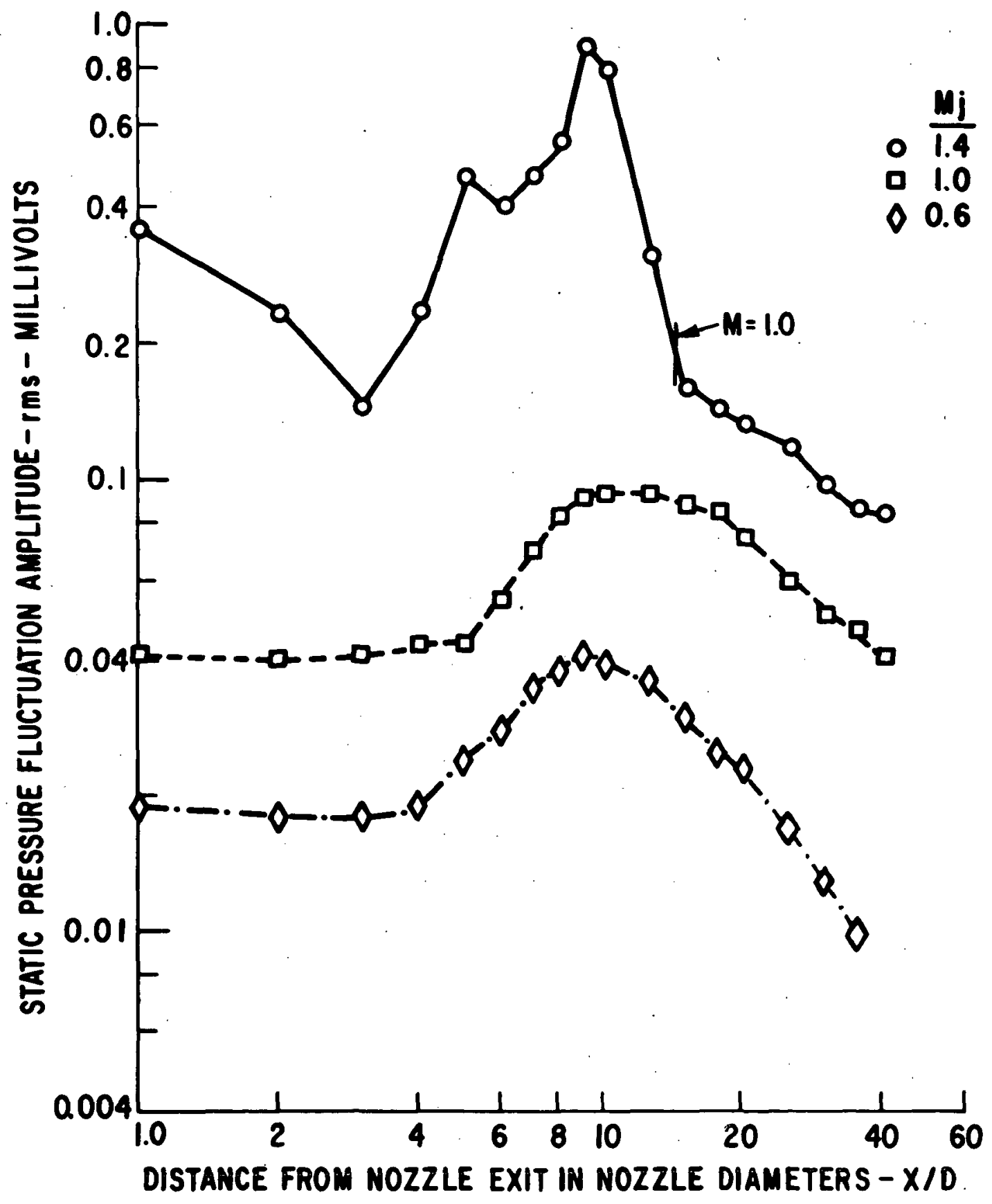

FIG.I3o AXIAL STATIC PRESSURE FLUCTUATIONS FOR 2 INCH CONVERGENT NOZZLE 


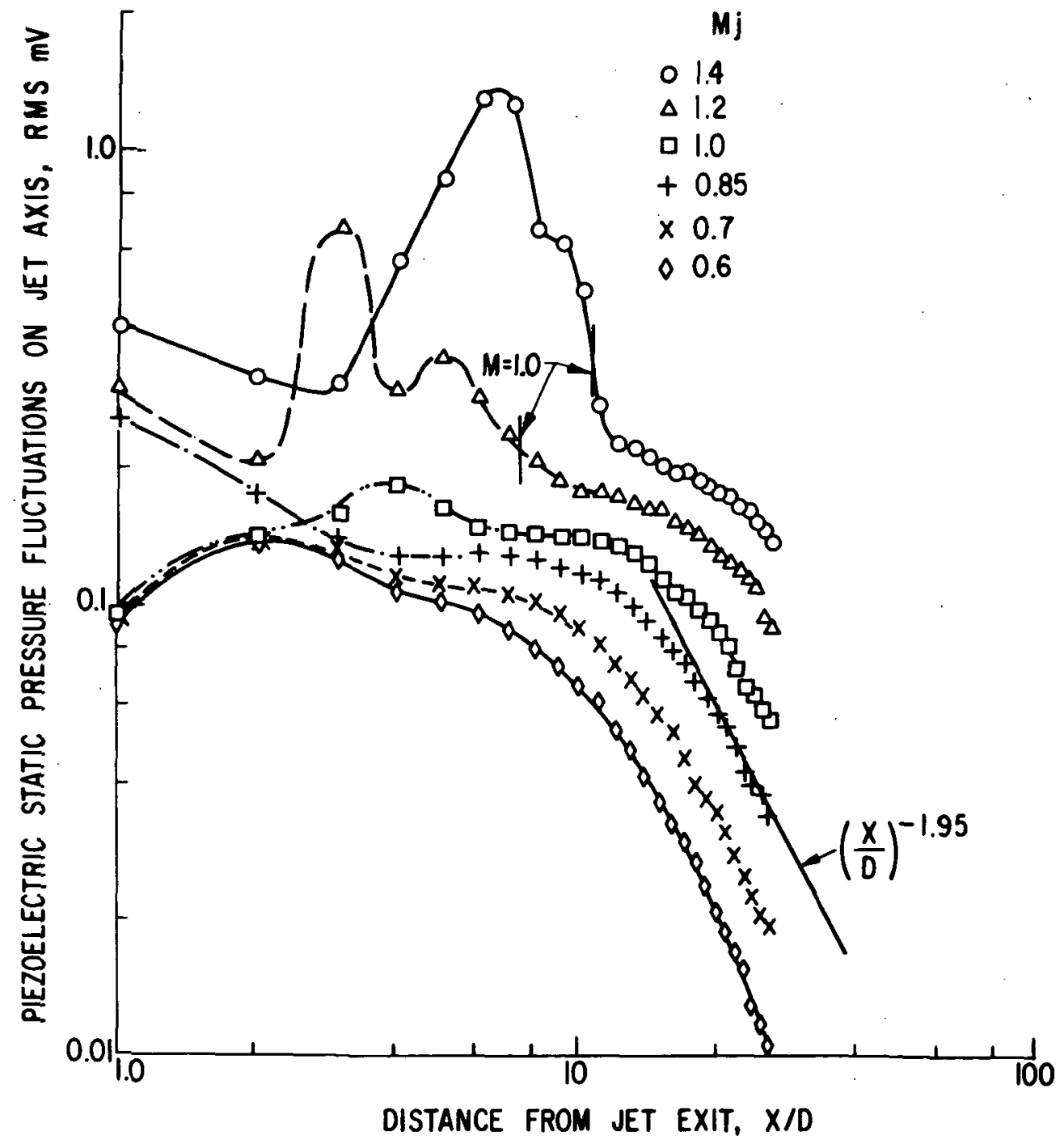

FIG. IBb VARIATION OF PIEZOELECTRIC STATIC PRESSURE FLUCTUATIONS ON JET AXIS WITH DISTANCE FROM JET EXIT FOR ONE INCH DIAMETER CONVERGENT NOZZLE 


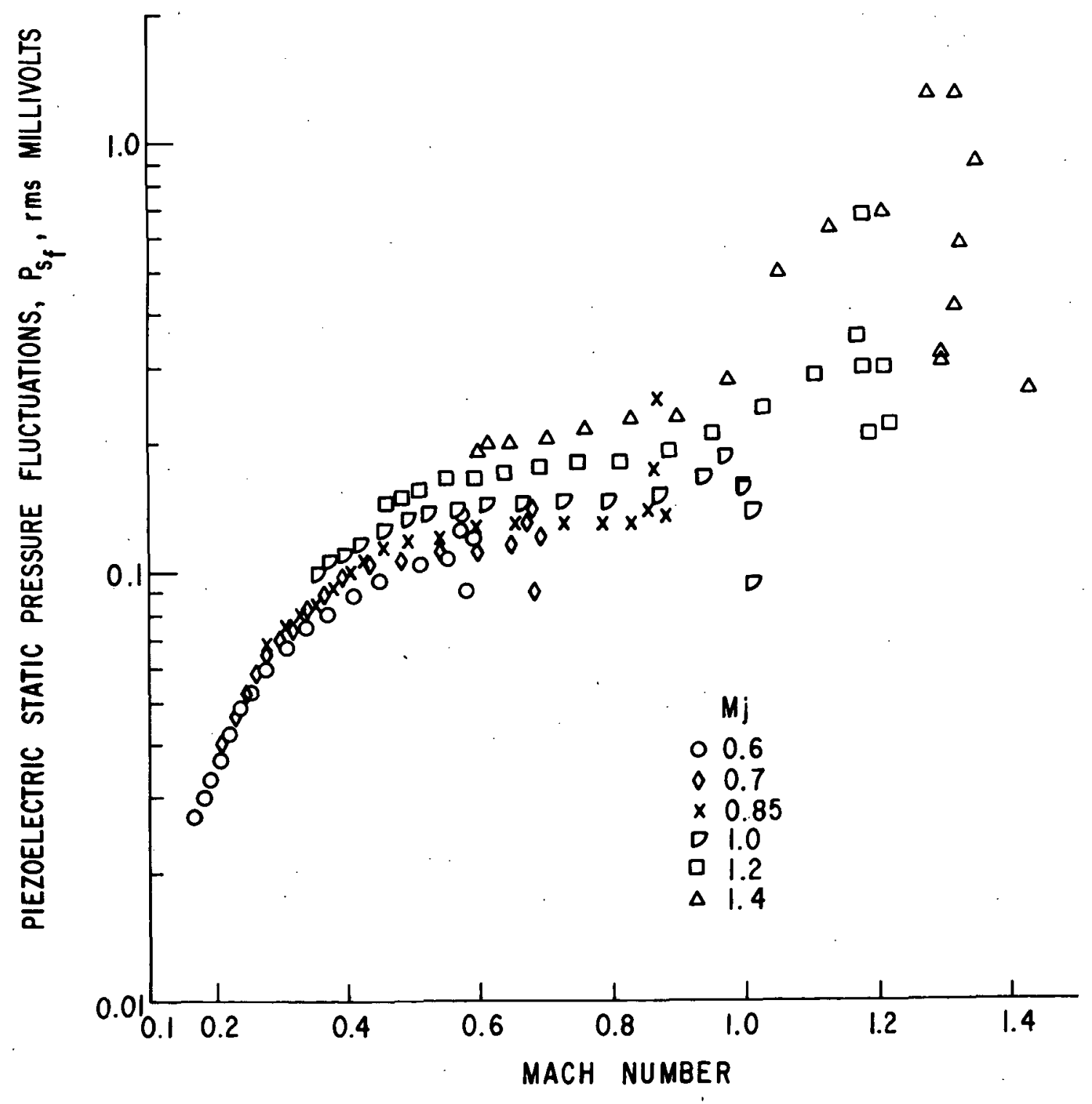

FIG. I3C VARIATION OF PIEZOELECTRIC STATIC PRESSURE FLUCTUATIONS ON JET AXIS AS FUNCTION OF LOCAL MACH NUMBER FOR ONE INCH DIAMETER CONVERGENT NOZZLE 

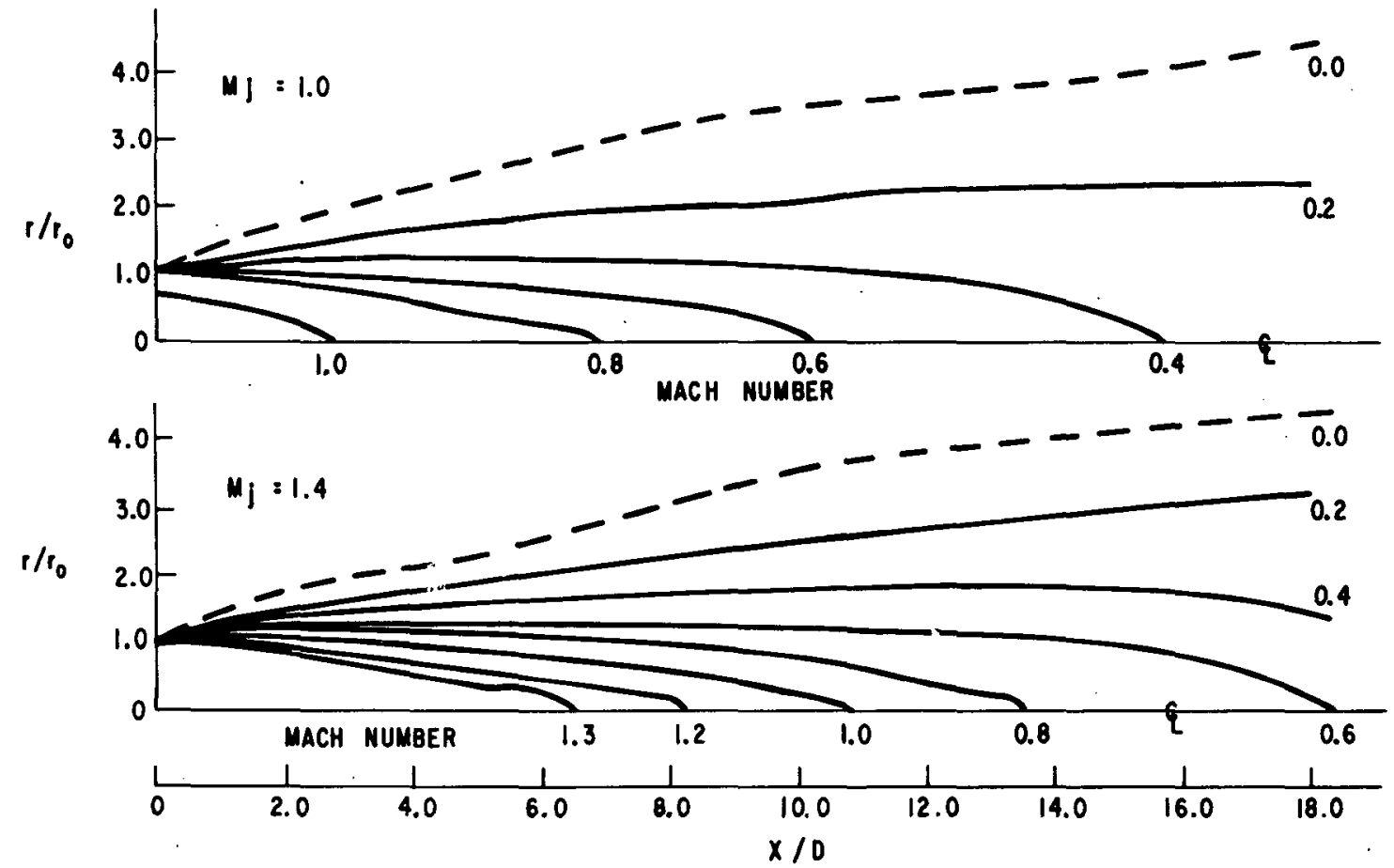

FIG. 14 CONSTANT MACH NUMBER CONTOURS IN THE FLOW FIELD FROM A ONE INCH CONVERGENT NOZZLE, Mj $\approx 1.0$ AND 1.4 


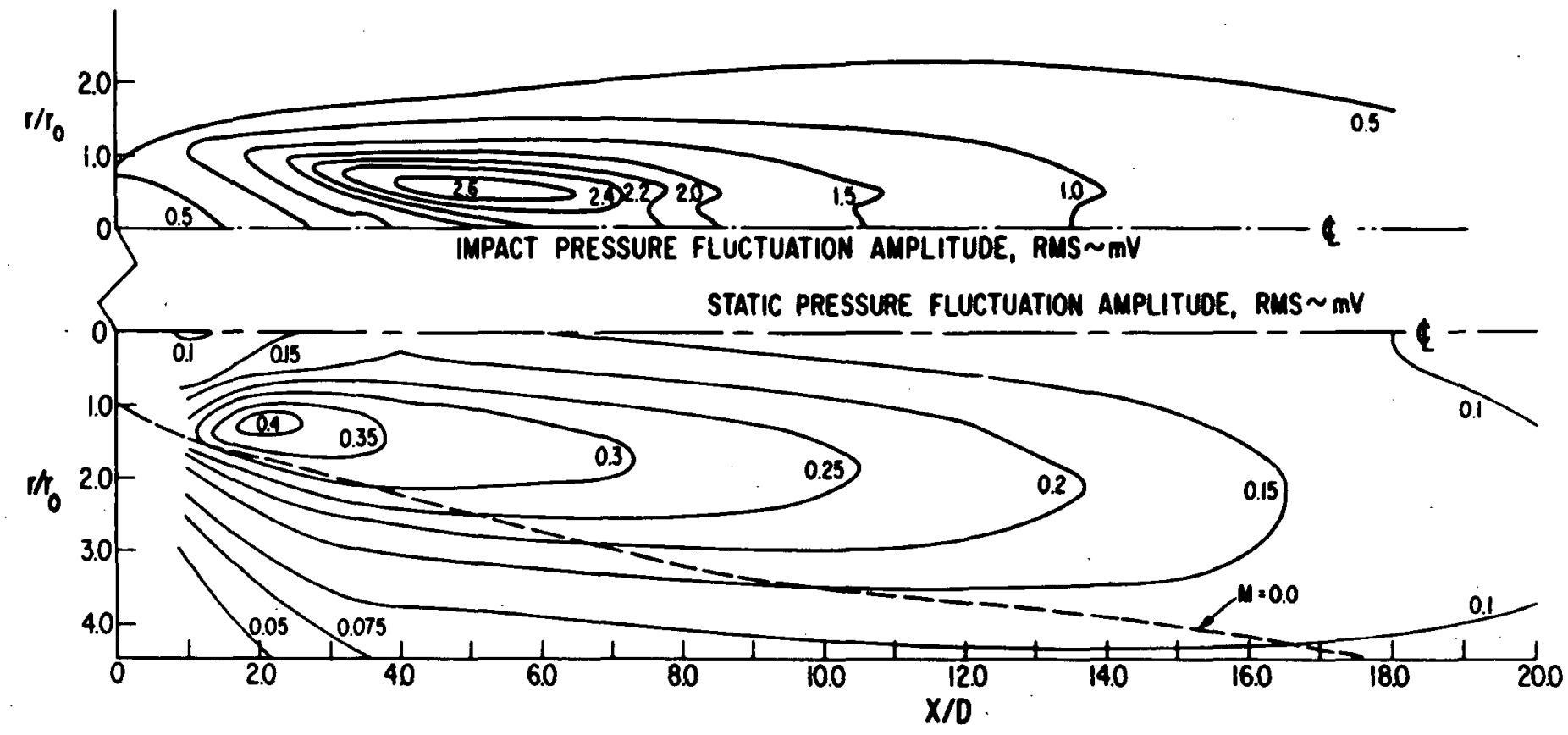

FIG.150 CONSTANT PIEZOELECTRIC IMPACT AND STATIC PRESSURE FLUCTUATION CONTOURS IN THE FLOW FIELD FROM A ONE INCH CONVERGENT NOZZLE, $m_{j}=1.0$ 


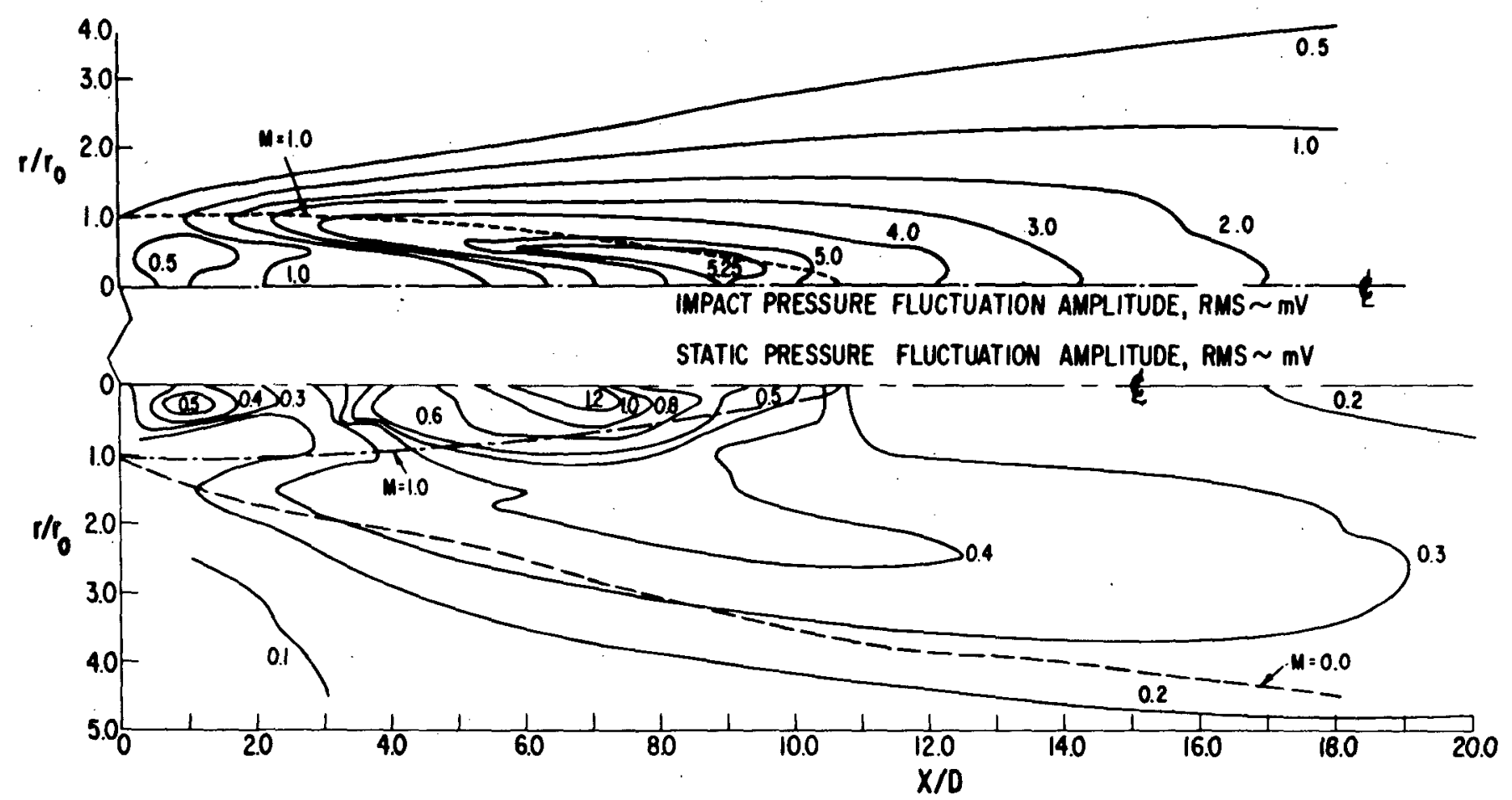

FIG. 15b CONSTANT PIEZOELECTRIC IMPACT AND STATIC PRESSURE FLUCTUATION CONTOURS IN THE FLOW FIELD FRON A ONE INCH CONVERGENT NOZZLE, $M j=1.4$ 
LIGHTHILL'S MODEL FOR $M_{j}<1$

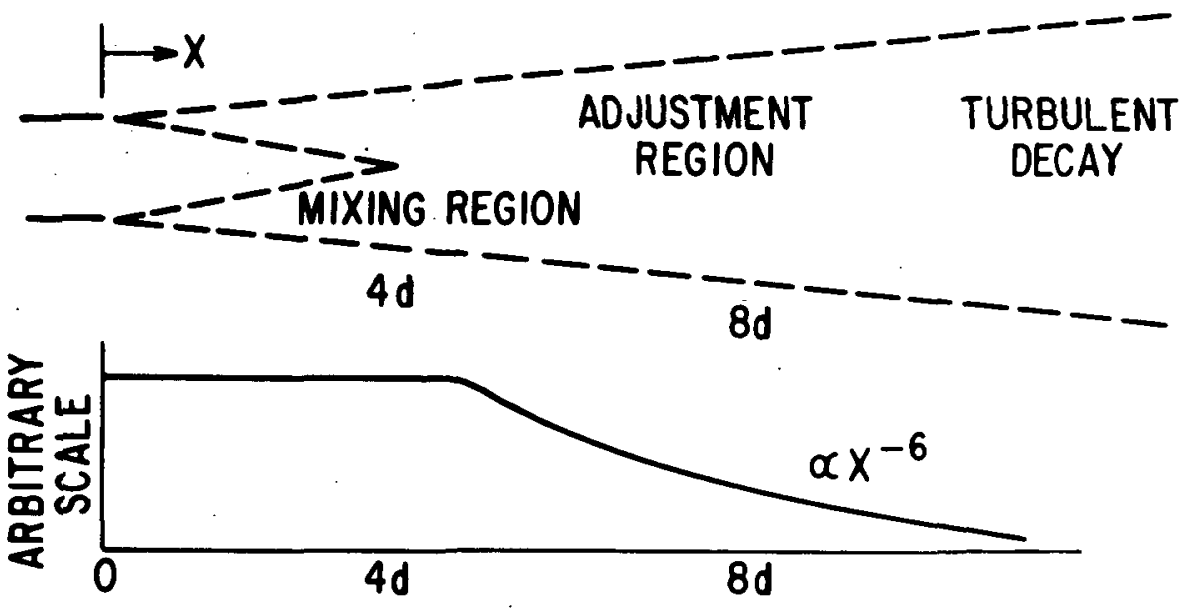

SUPERSONIC JET $M_{j}=2.49$
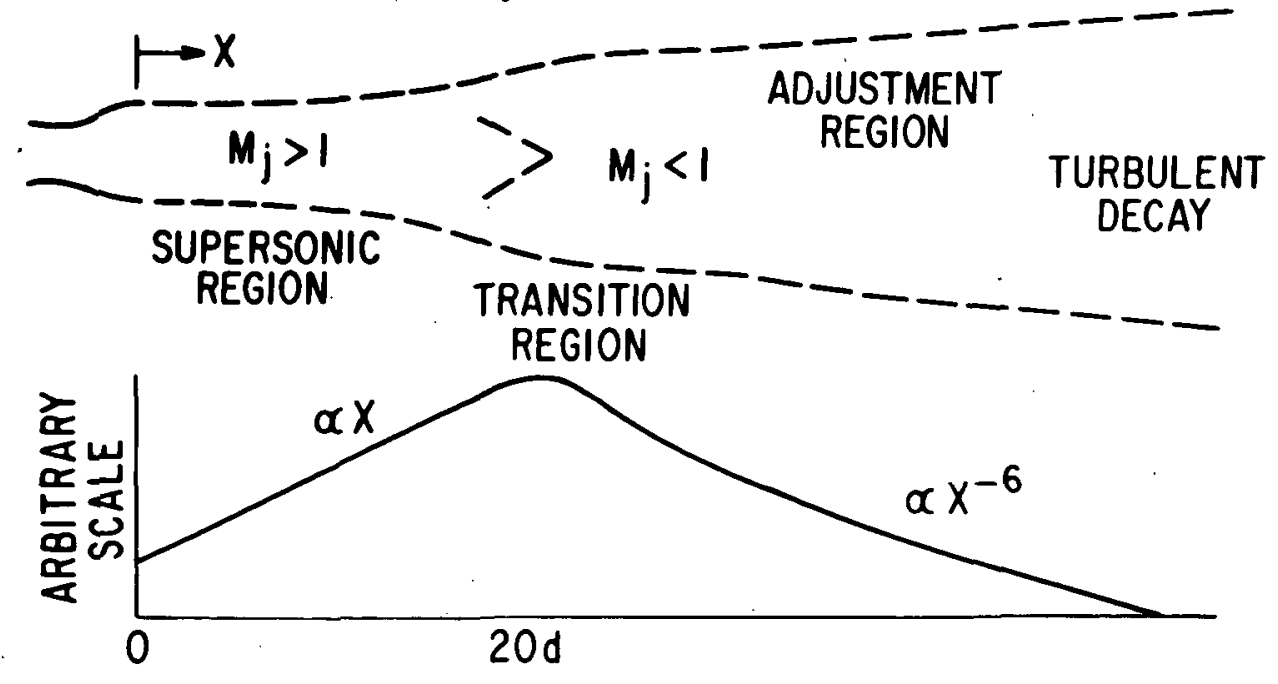

FIG.I6 COMPARISON OF SUBSONIC AND SUPERSONIC JET AERODYNAMIC AND ACOUSTIC CHARACTERISTICS. 


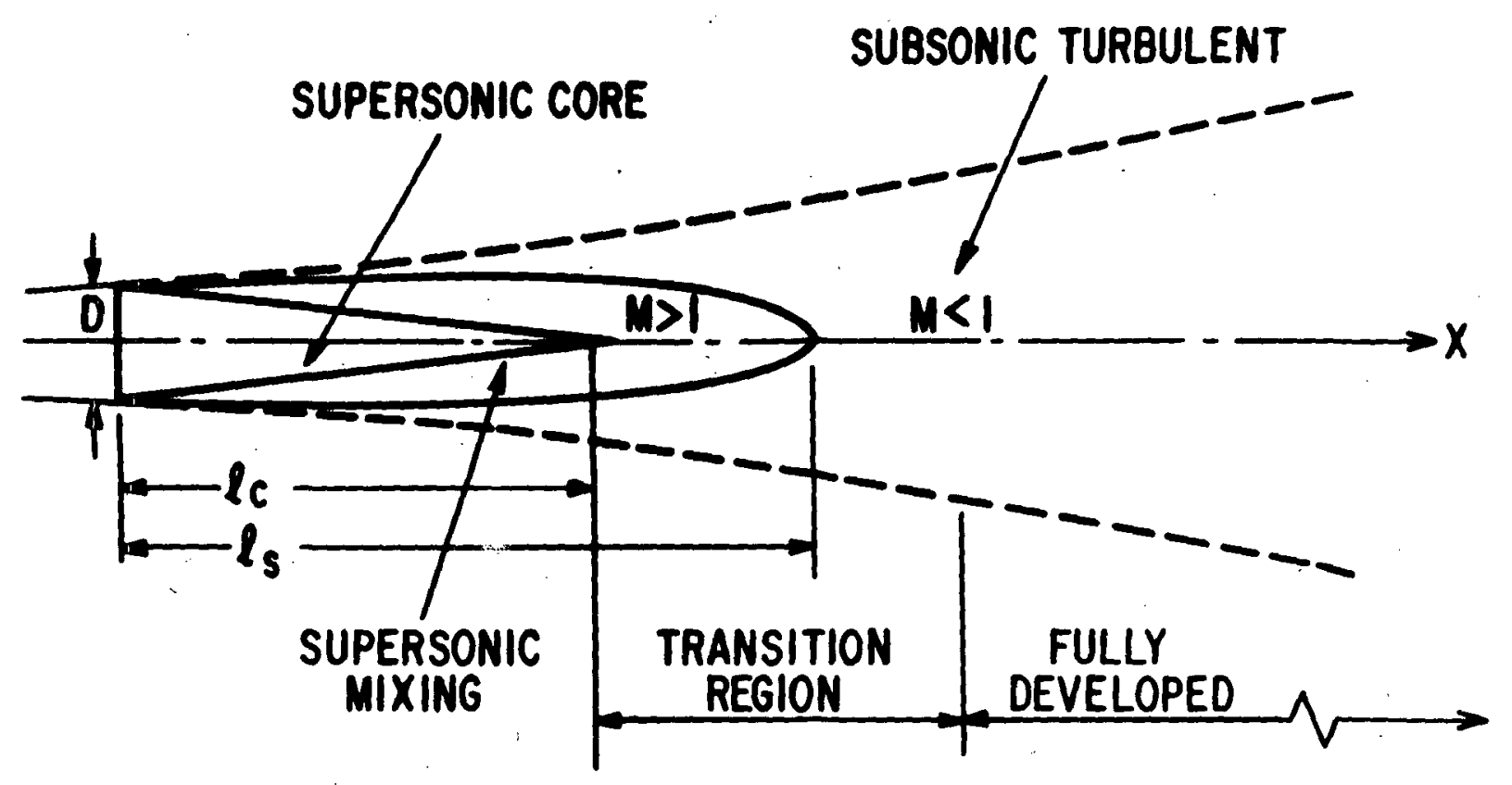

FIG. I7 PARALLEL FLOW SUPERSONIC JET EXPANDED TO AMBIENT PRESSURE 


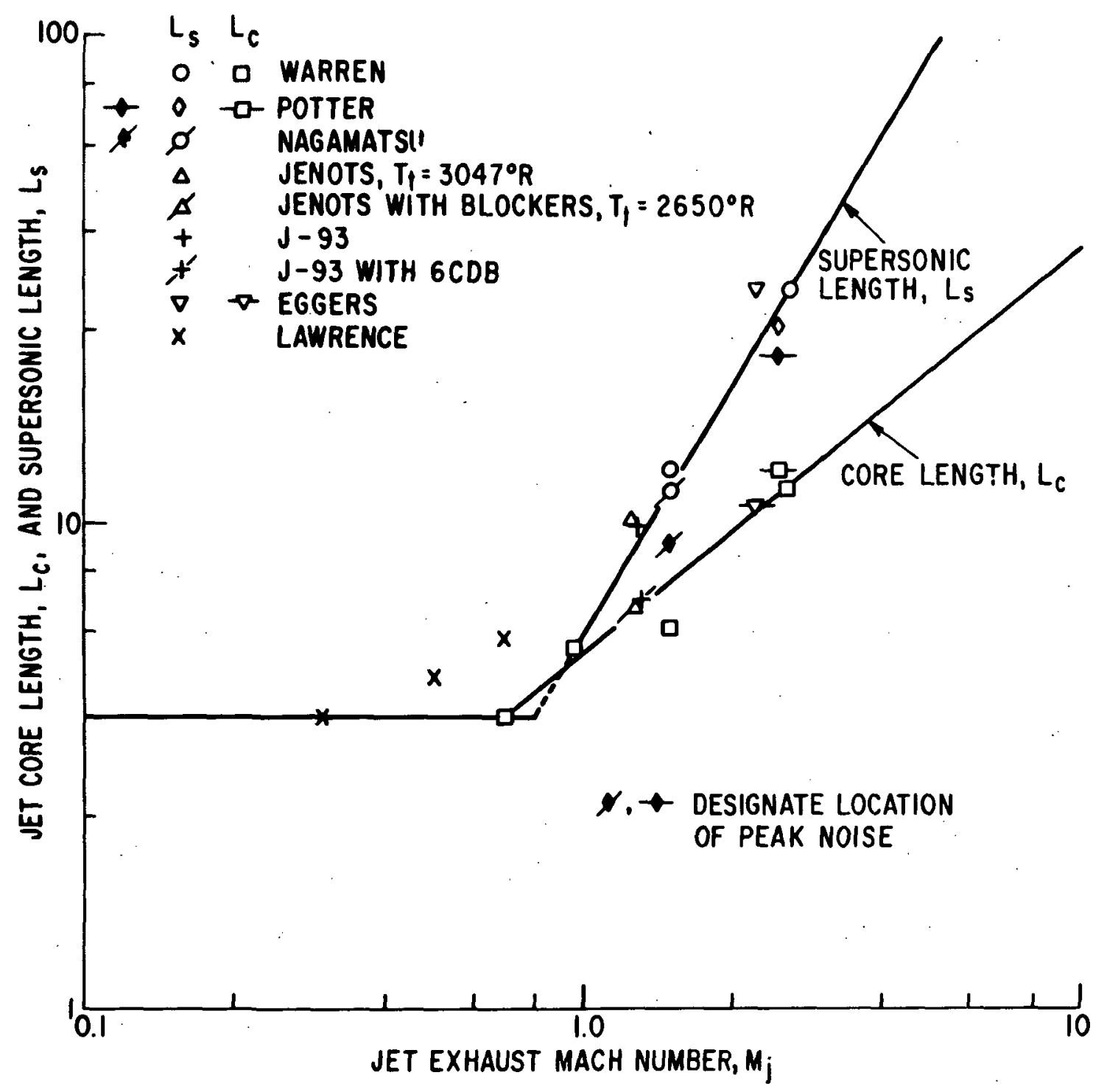

FIG.18 JET CORE LENGTH, AND SUPERSONIC LENGTH, AS FUNCTION OF JET MACH NUMBER. 


$$
\text { B. }
$$




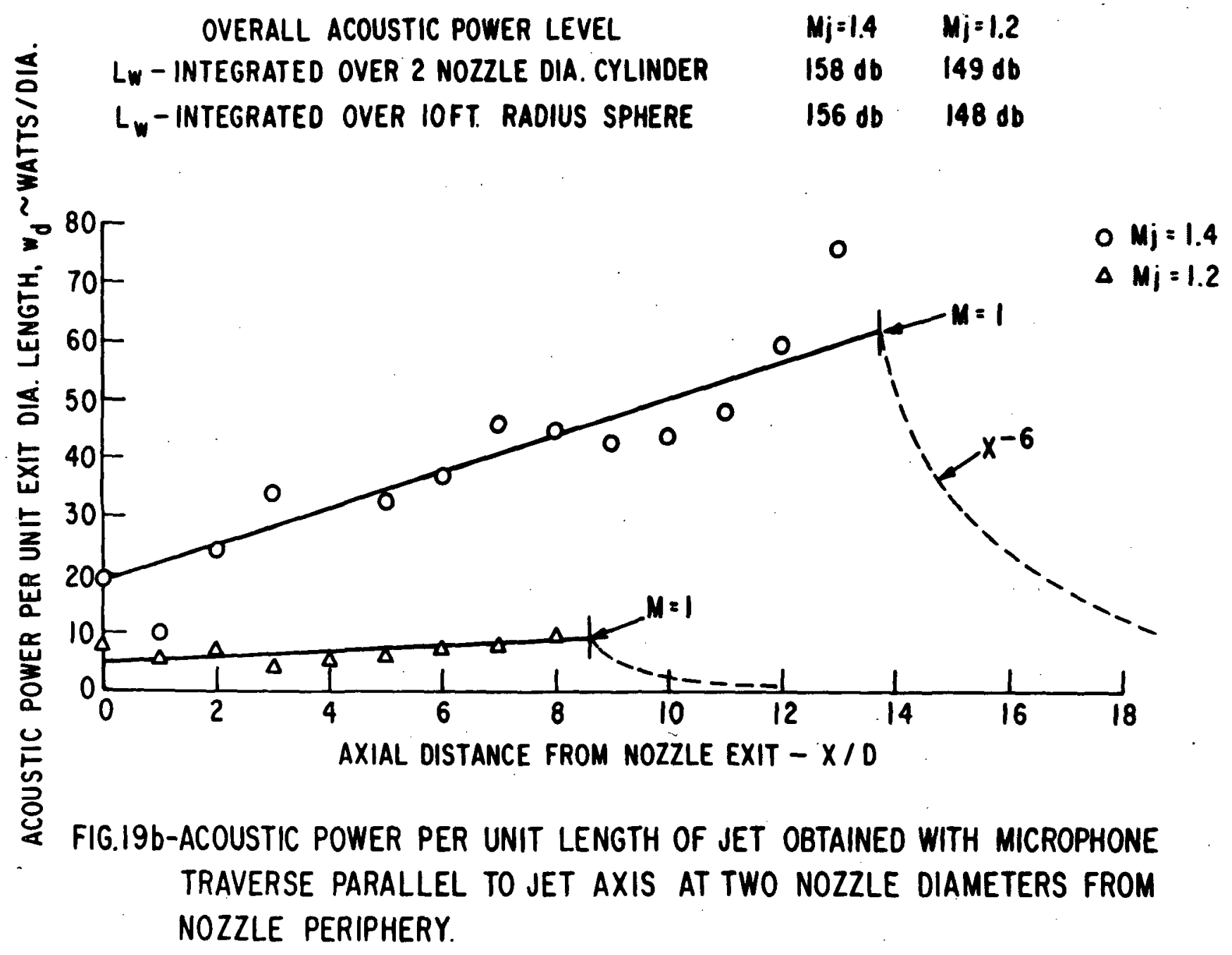




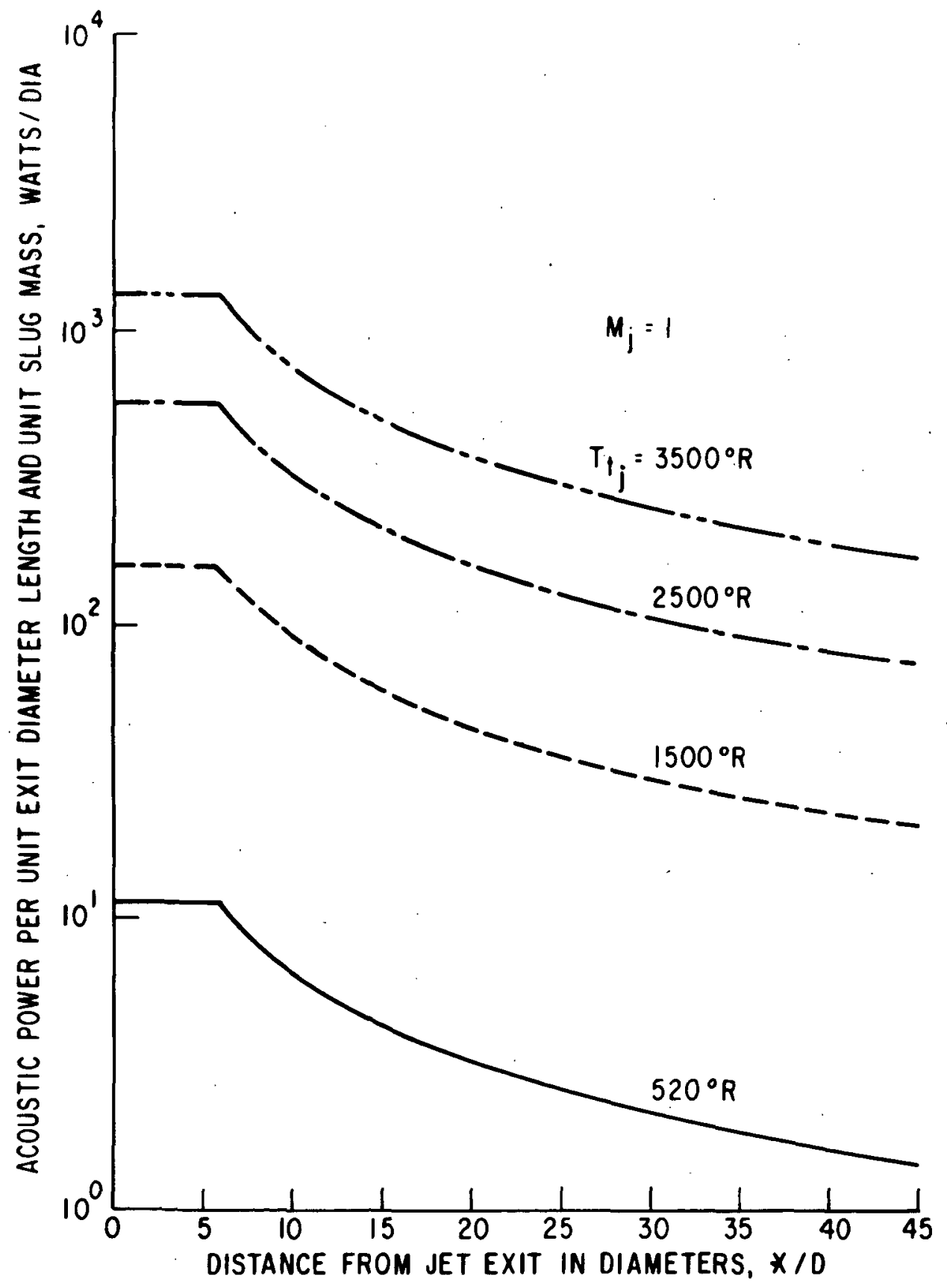

FIG.20a ACOUSTIC POWER GENERATED PER UNIT LENGTH OF THE JET FOR JET MACH NUMBER OF 1.0 . 


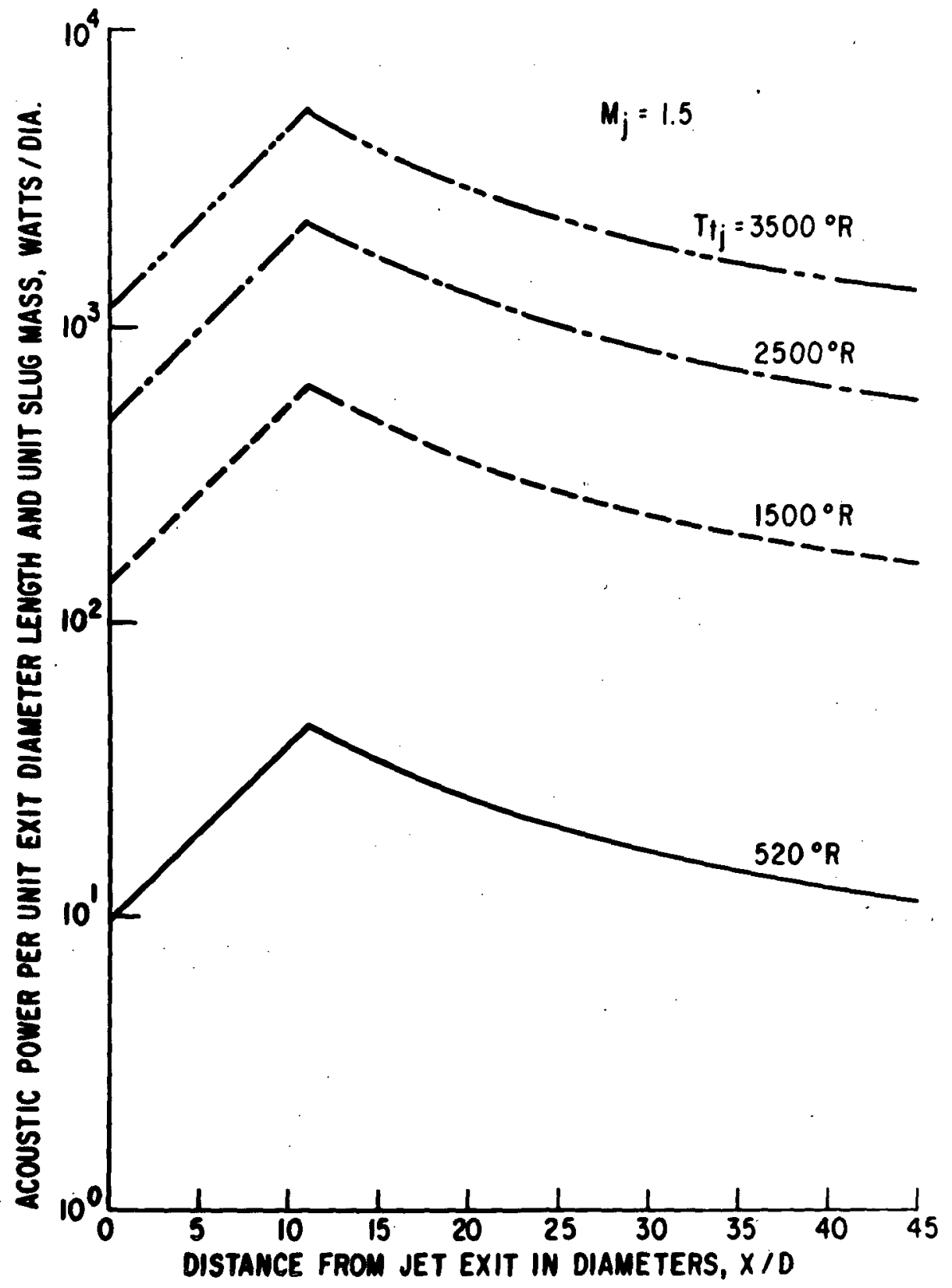

FIG.2Ob ACOUSTIC POWER GENERATED PER UNIT LENGTH OF THE JET FOR JET MACH NUMBER OF I.5. 


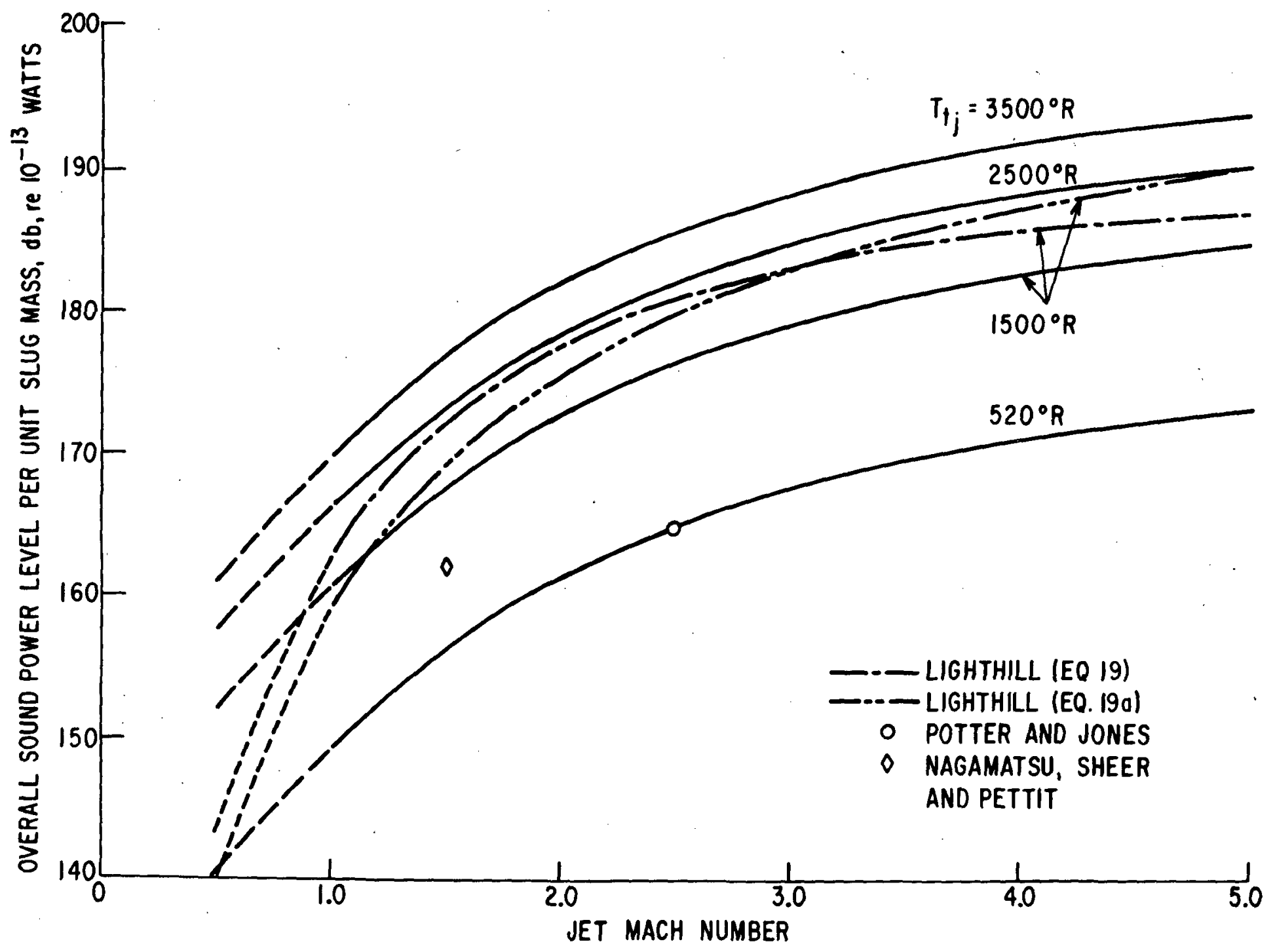

FIG.21 OVERALL SOUND POWER LEVEL IN db PER UNIT SLUG MASS AS FUNCTION OF JET MACH NUMBER. 


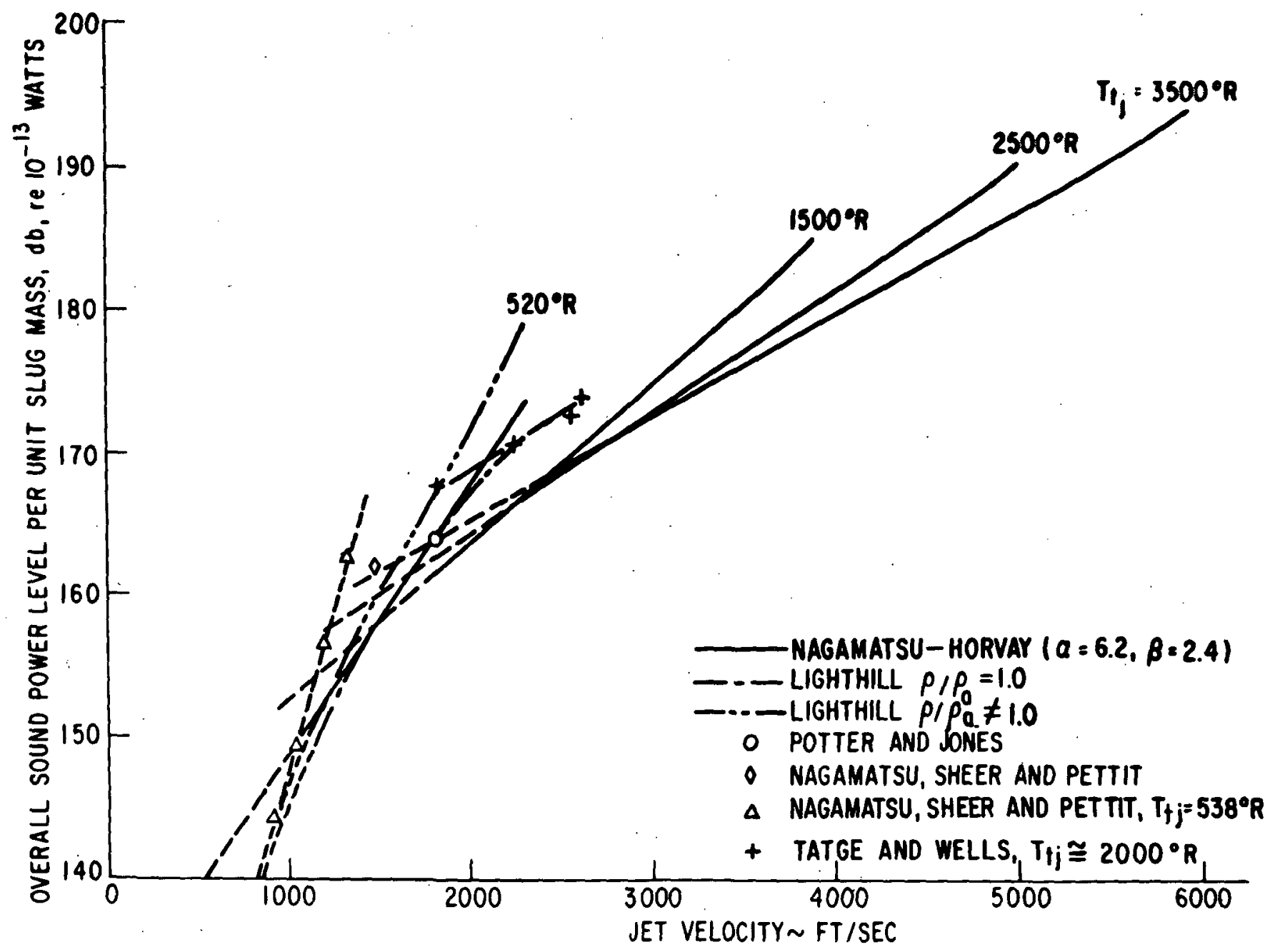

FIG.2Ib - OVERALL SOUNO POWER LEVEL IN db PER UNIT SLUG MASS AS FUNCTION OF JET VELOCITY. 


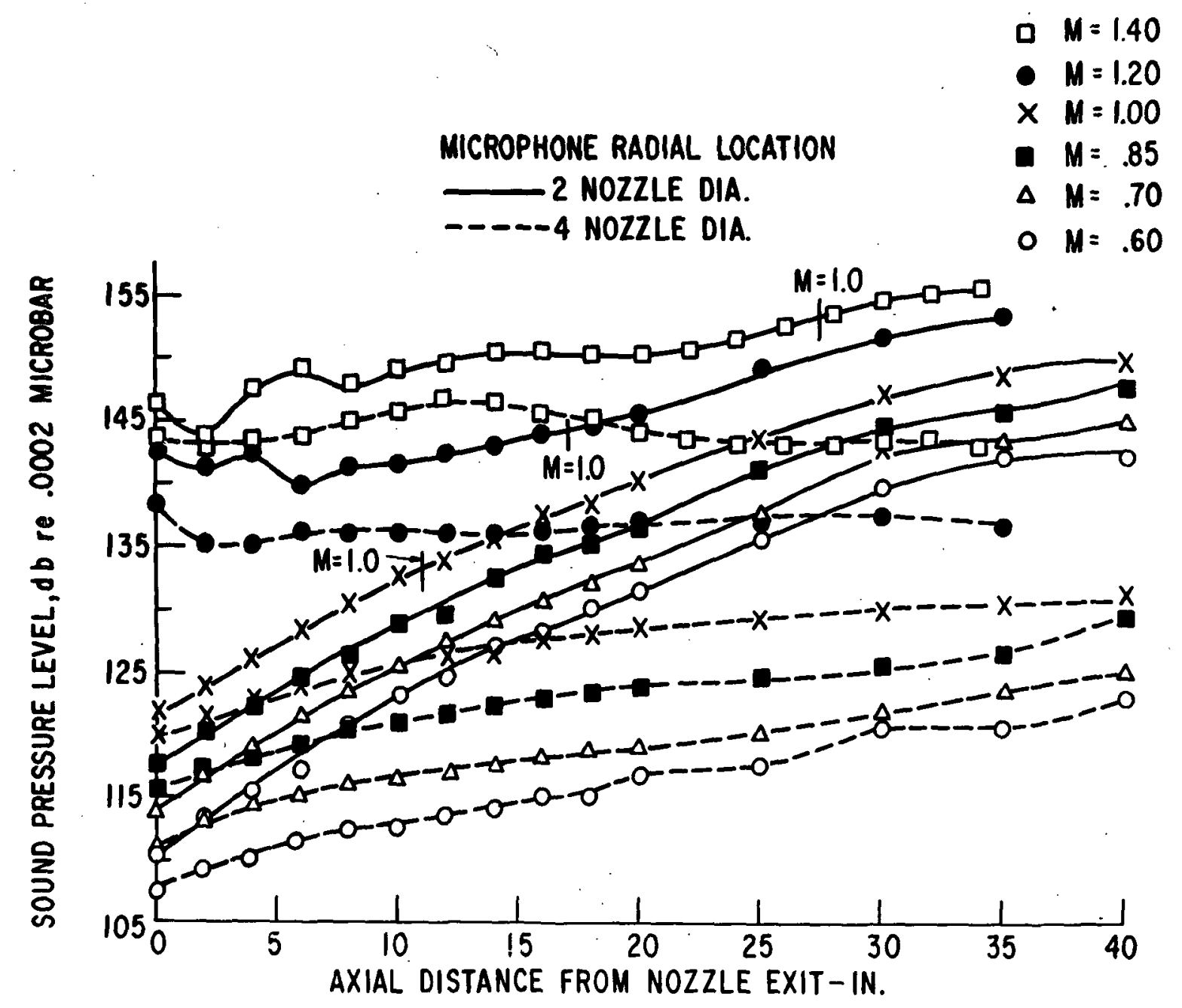

FIG.22 - SOUND PRESSURE LEVEL VARIATION PARALLEL TO JET AXIS AT 2 AND 4 DIAMETERS FROM NOZZLE EXIT. 


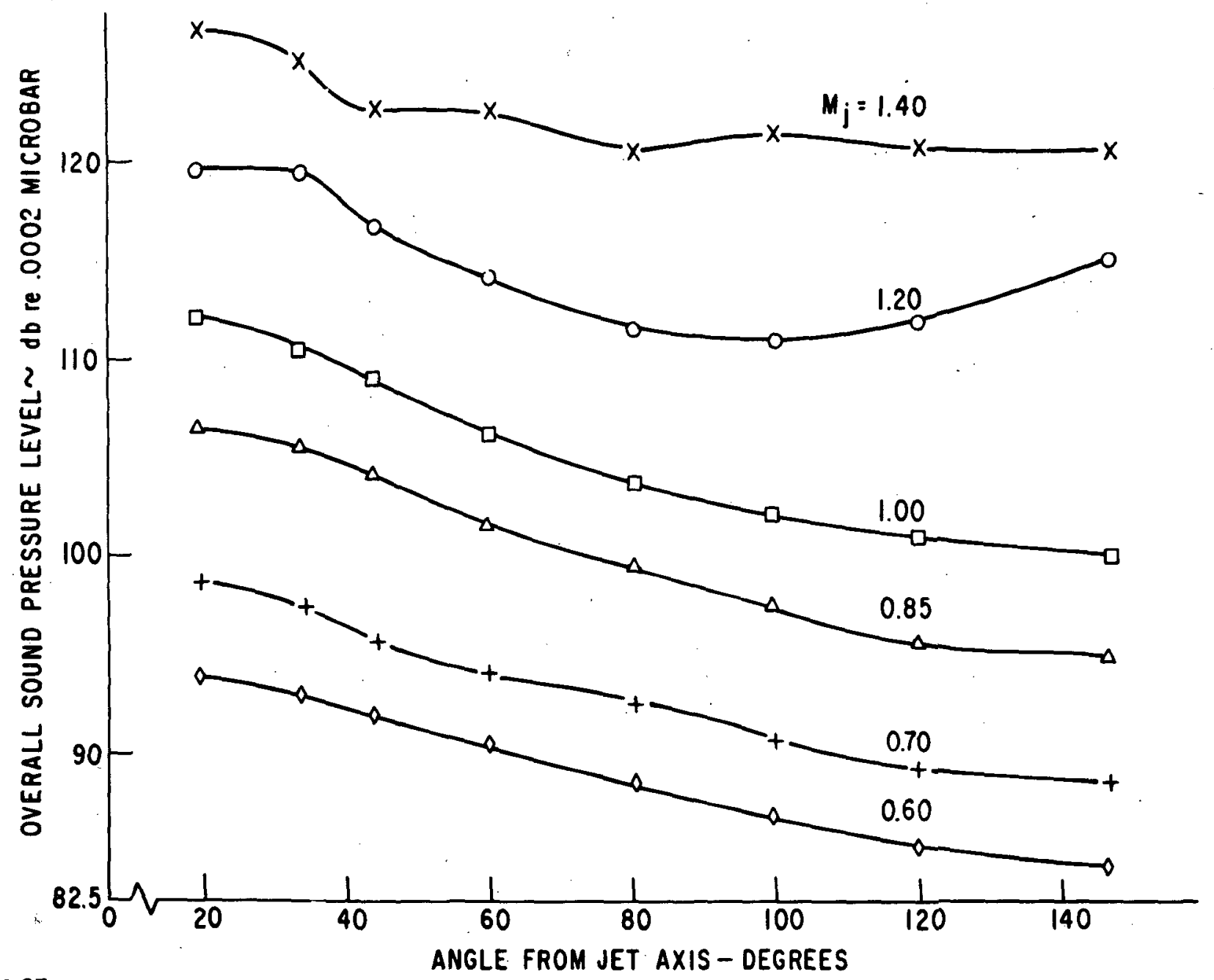

FIG.23- OVERALL SOUND PRESSURE LEVEL AS A. FUNCTION OF ANGULAR POSITION FROM JET AXIS FOR OIFFERENT JET MACH NUMBERS. 


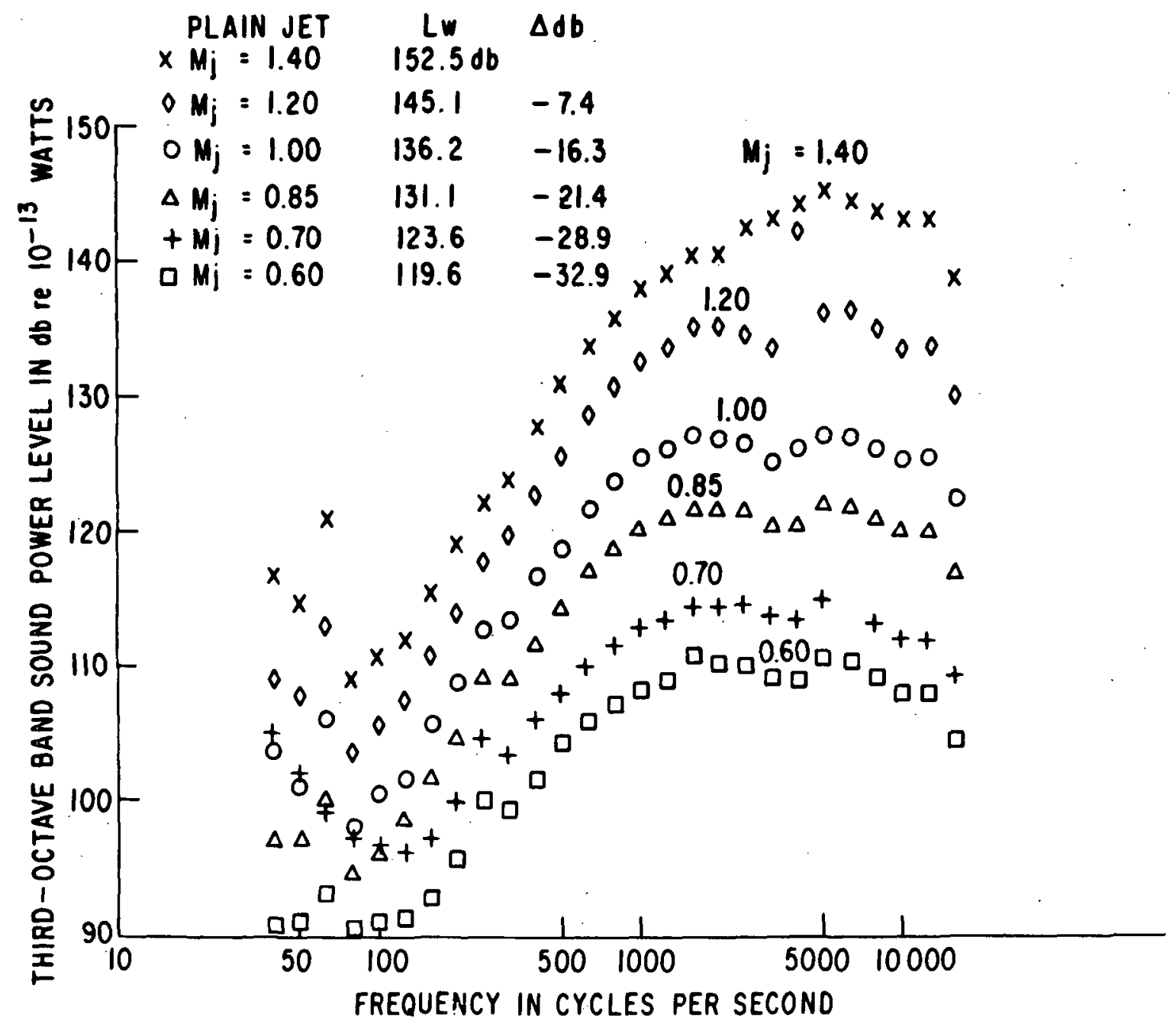

FIG.24COMPARISON OF SOUND POWER SPECTRA FOR PLAIN CONVERGENT JET AT VARIOUS MACH NUMBERS. 


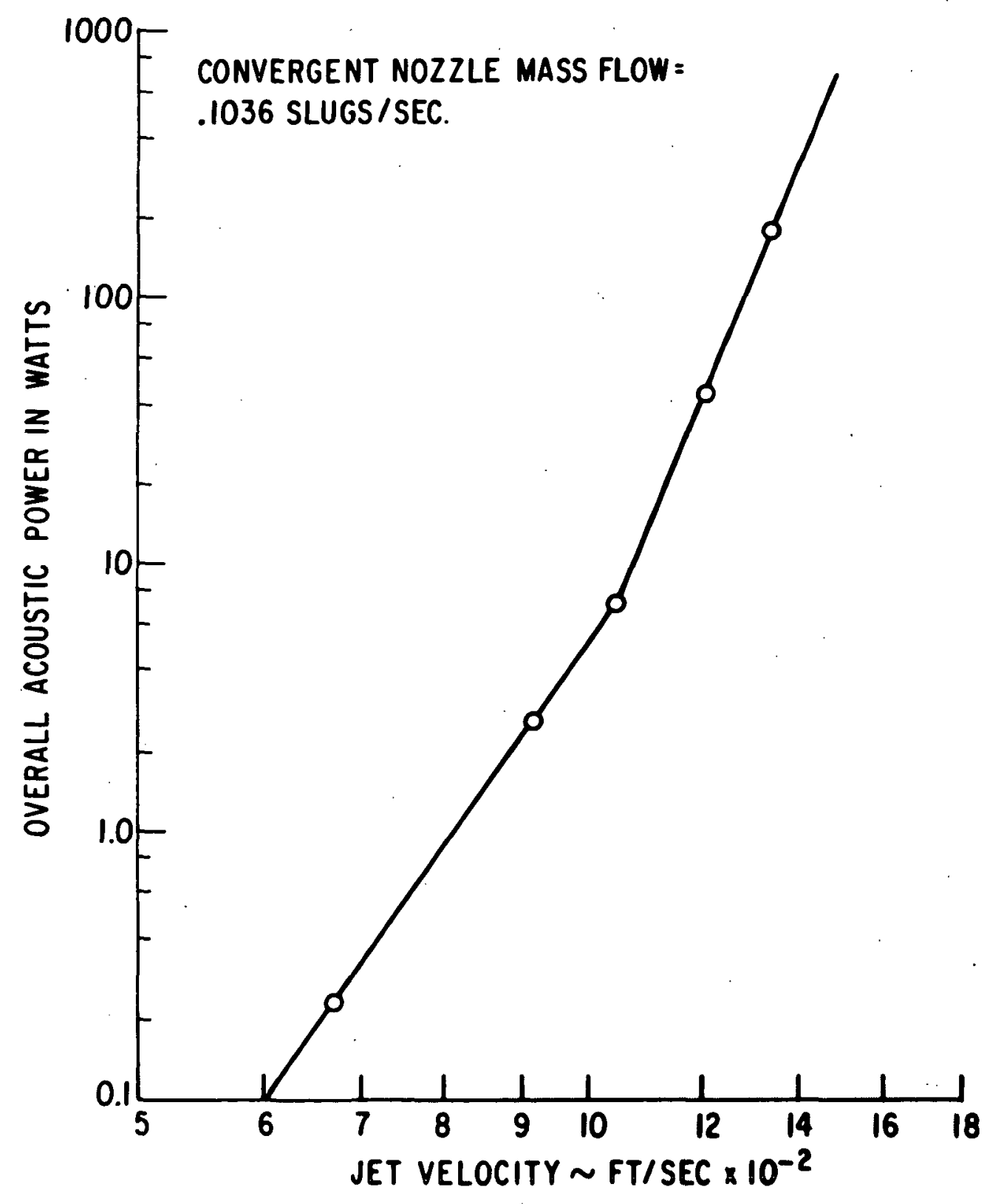

FIG. 25 OVERALL SOUND POWER LEVEL IN WATTS FOR CONSTANT MASS FLOW AS FUNCTION OF JET VELOCITY. 


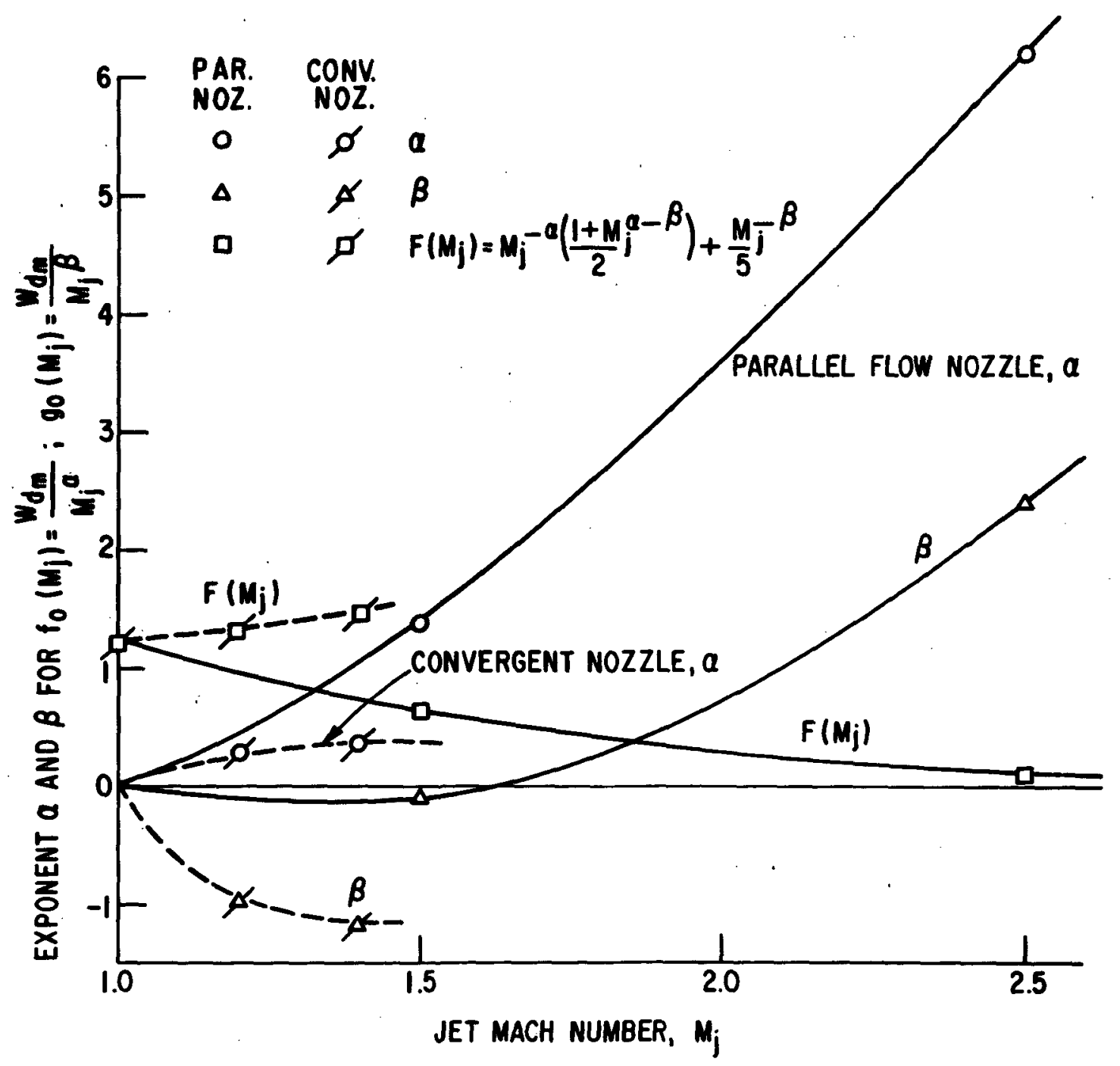

FIG.26-VALUES OF EXPONENTS $a$ AND $\beta$ OF NAGAMATSU - HORVAY SUPERSONIC JET NOISE THEORY AS FUNCTION OF JET MACH NUMBER FOR PARALLEL FLOW AND CONVERGENT NOZZLE WITH $T_{f j}=520^{\circ} R$. 


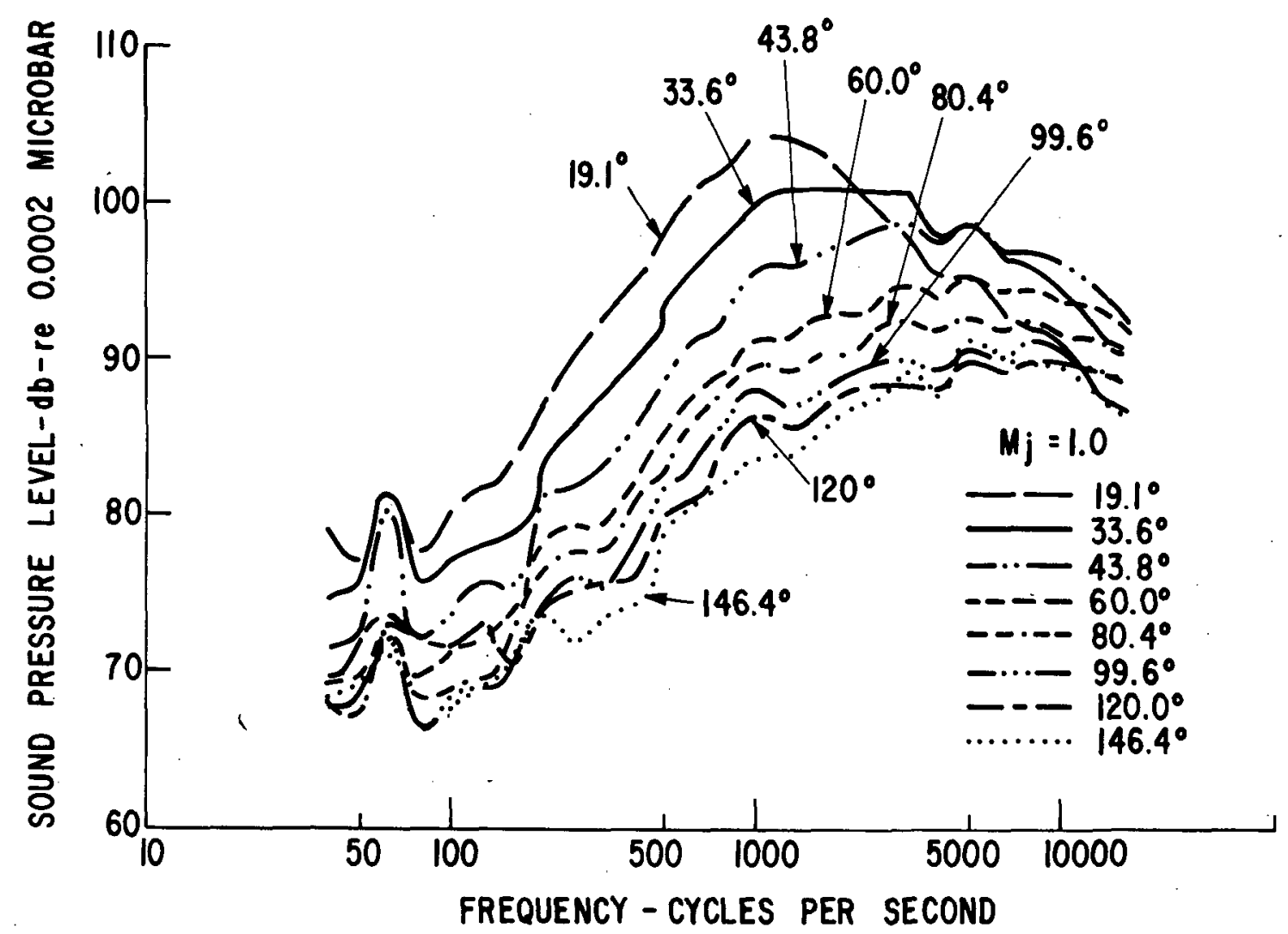

FIG. 27a SOUND PRESSURE LEVEL VS FREQUENCY AT 8 ANGULAR POSITIONS FROM JET AXIS FOR 2 INCH DIAMETER CONVERGENT NOZZLE, $M_{j}=1.0$ 


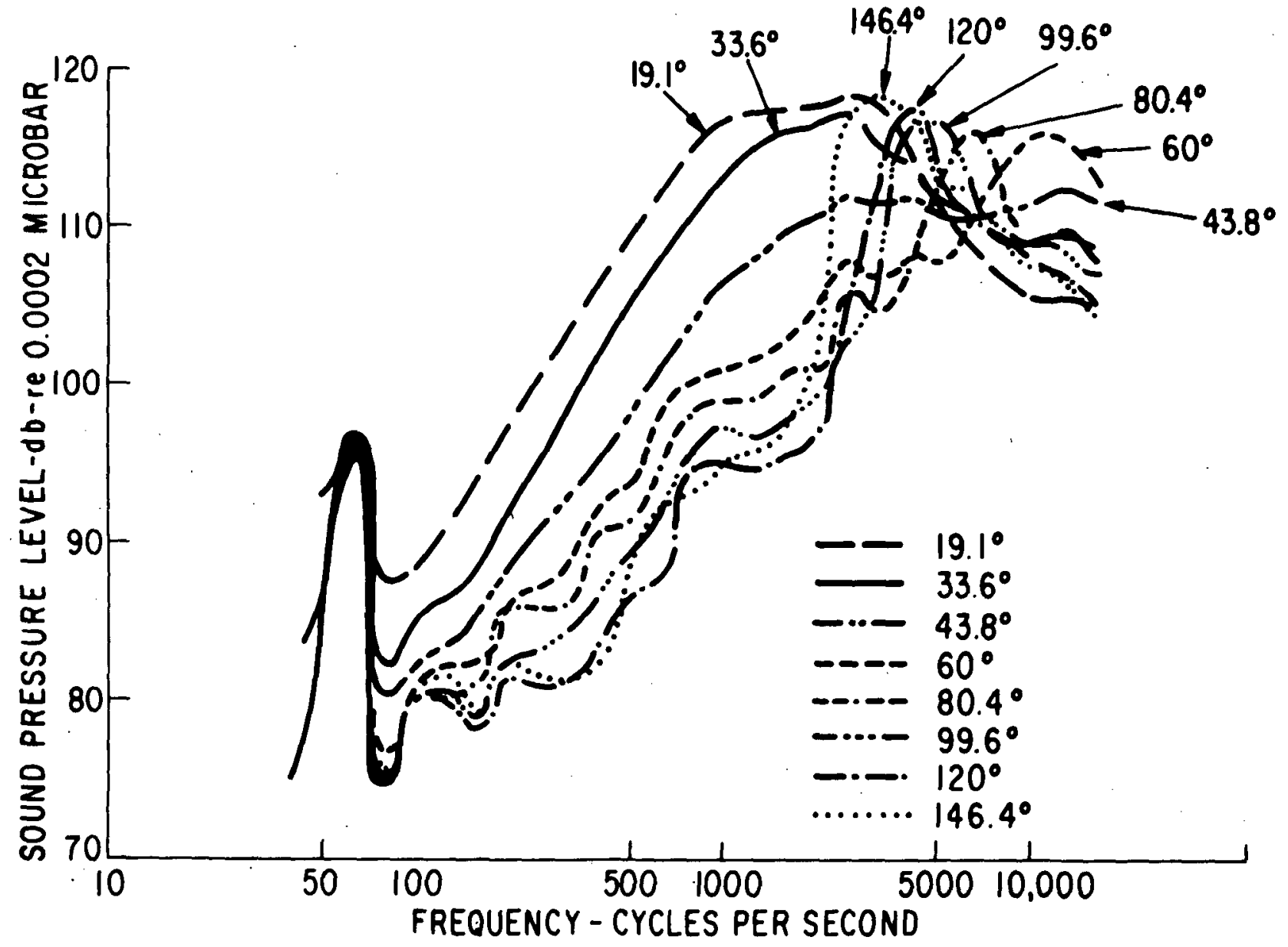

FIG.27 b SOUND PRESSURE LEVEL VS FREQUENCY AT 8 ANGULAR POSITIONS FROM JET AXIS FOR 2 INCH DIAMETER CONVERGENT NOZZLE, $M j=1.4$ 


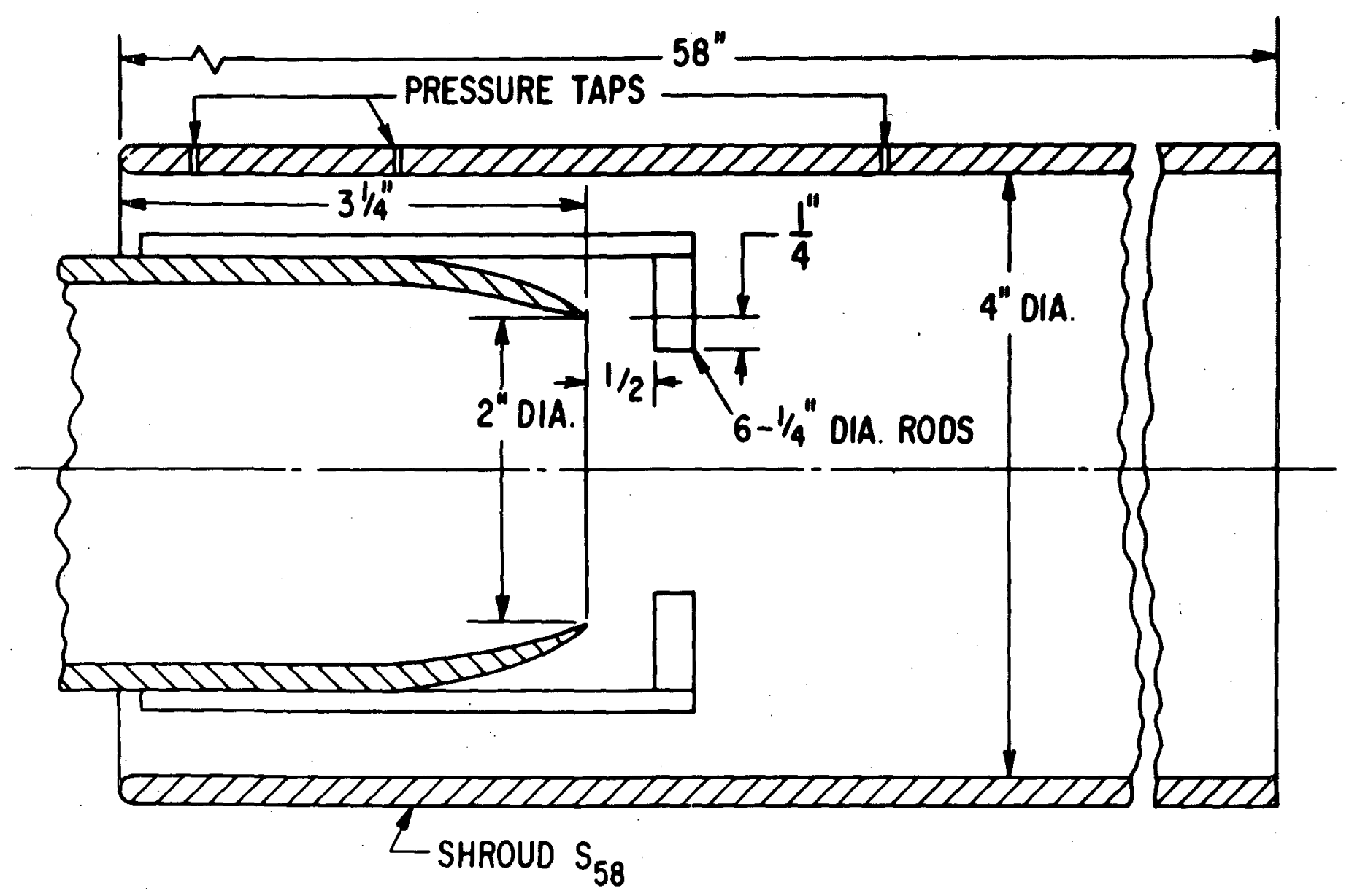

FIGURE 28 SKETCH OF NOZZLE, SHROUD, AND RODS. 


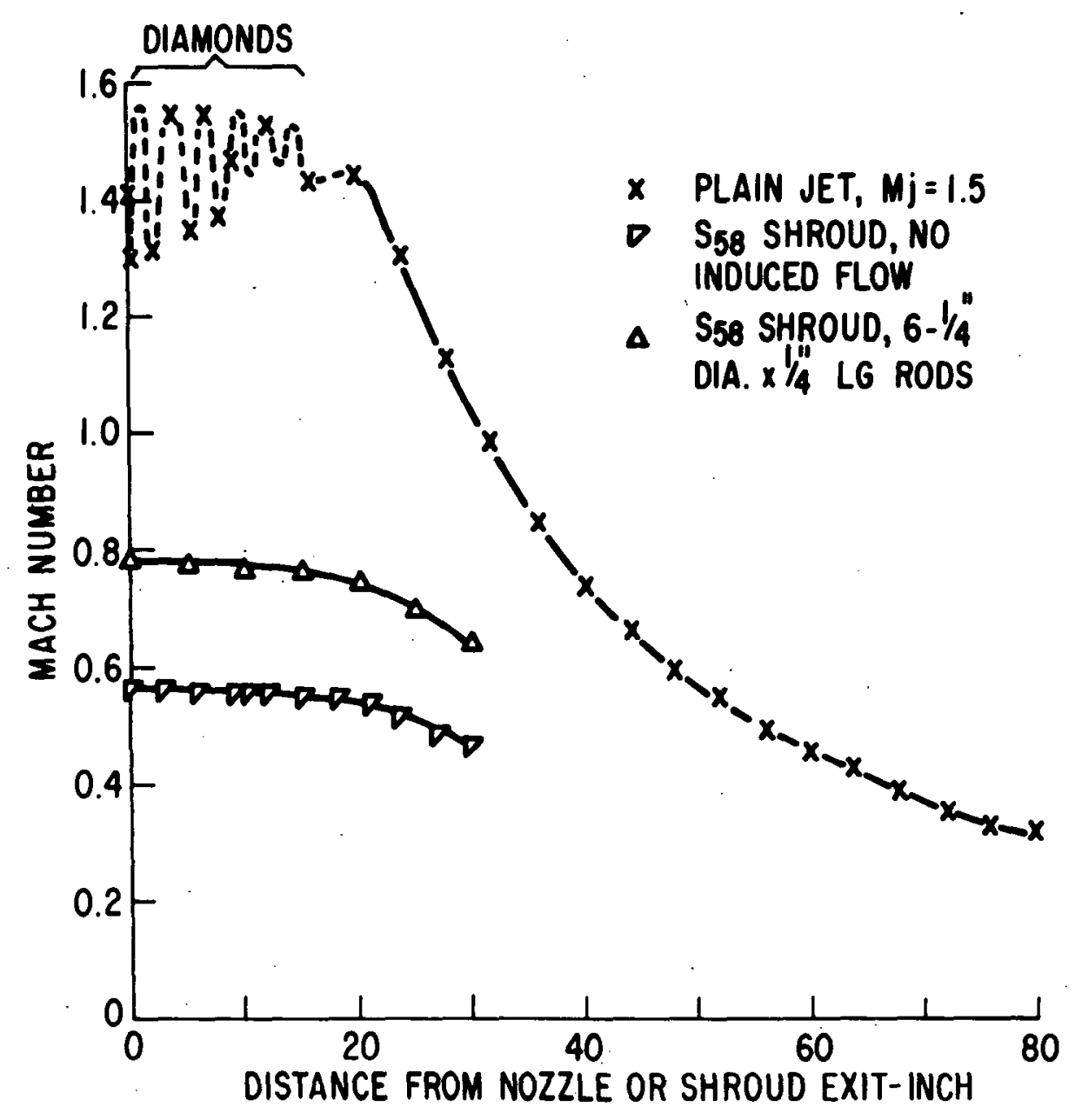

FIG.290 AXIAL MACH NUMBER DISTRIBUTION FOR 2 INCH DIAMETER CONVERGENT NOZZLE WITH AND WITHOUT SHROUD AND ROOS 


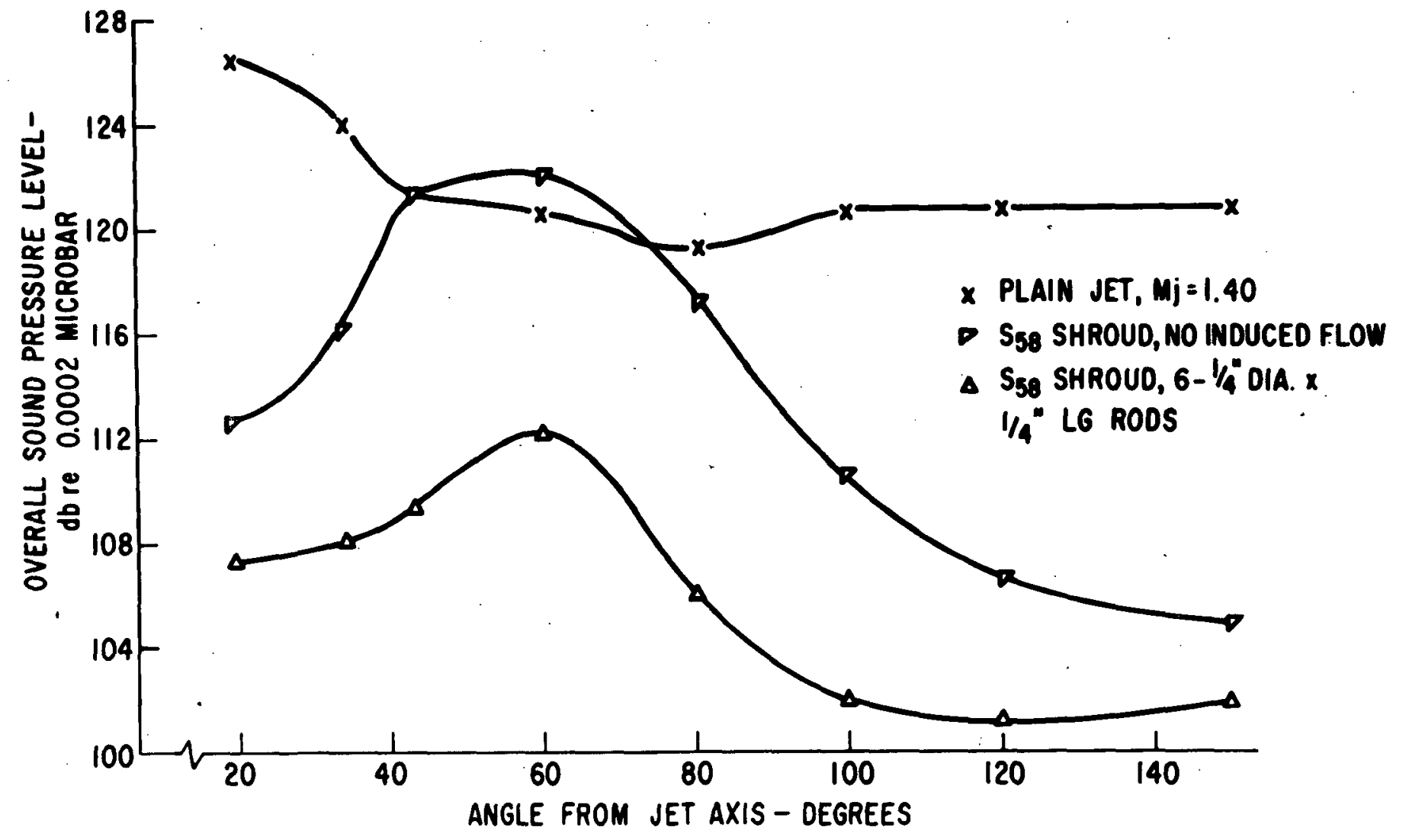

FIG. 29b OVERALL SOUND PRESSURE LEVEL AS FUNCTION OF ANGULAR POSITION FROM JET AXIS FOR 2 INCH DIAMETER CONVERGENT NOZZLE WITH AND WITHOUT SHROUD AND RODS 


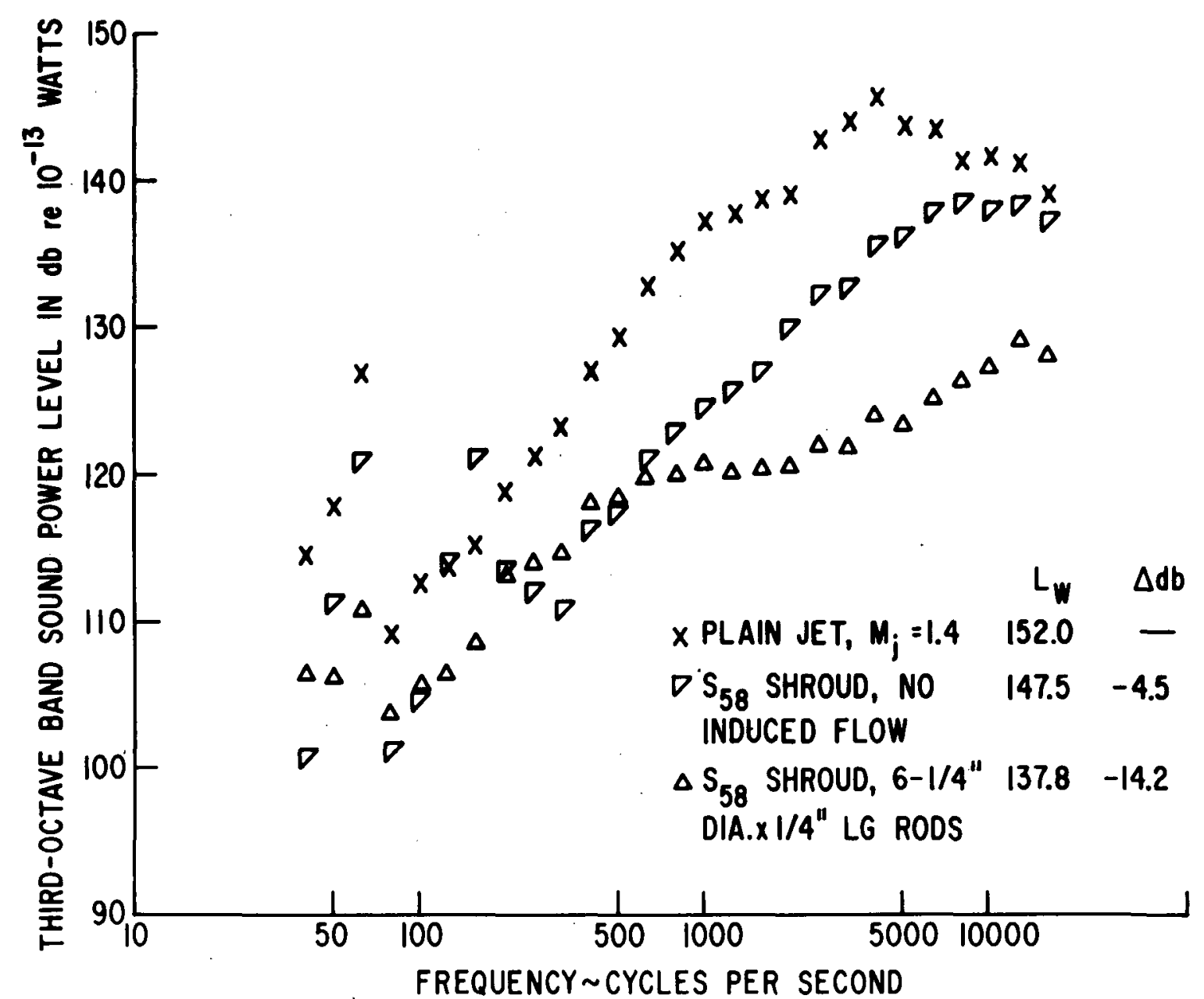

FIG.29c SOUND POWER LEVEL SPECTRA FOR 2 INCH DIAMETER CONVERGENT NOZZLE WITH AND WITHOUT SHROUD AND RODS 


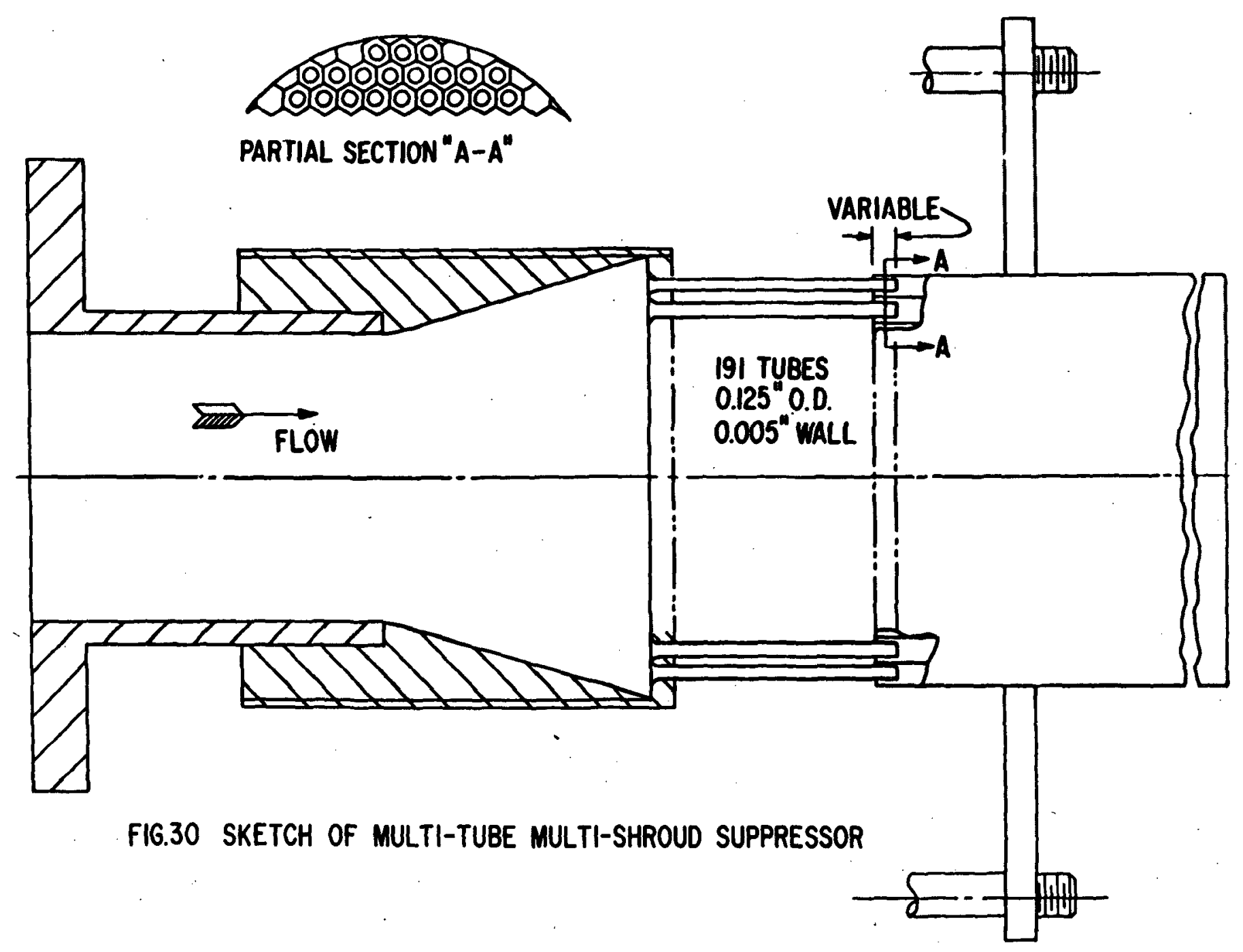




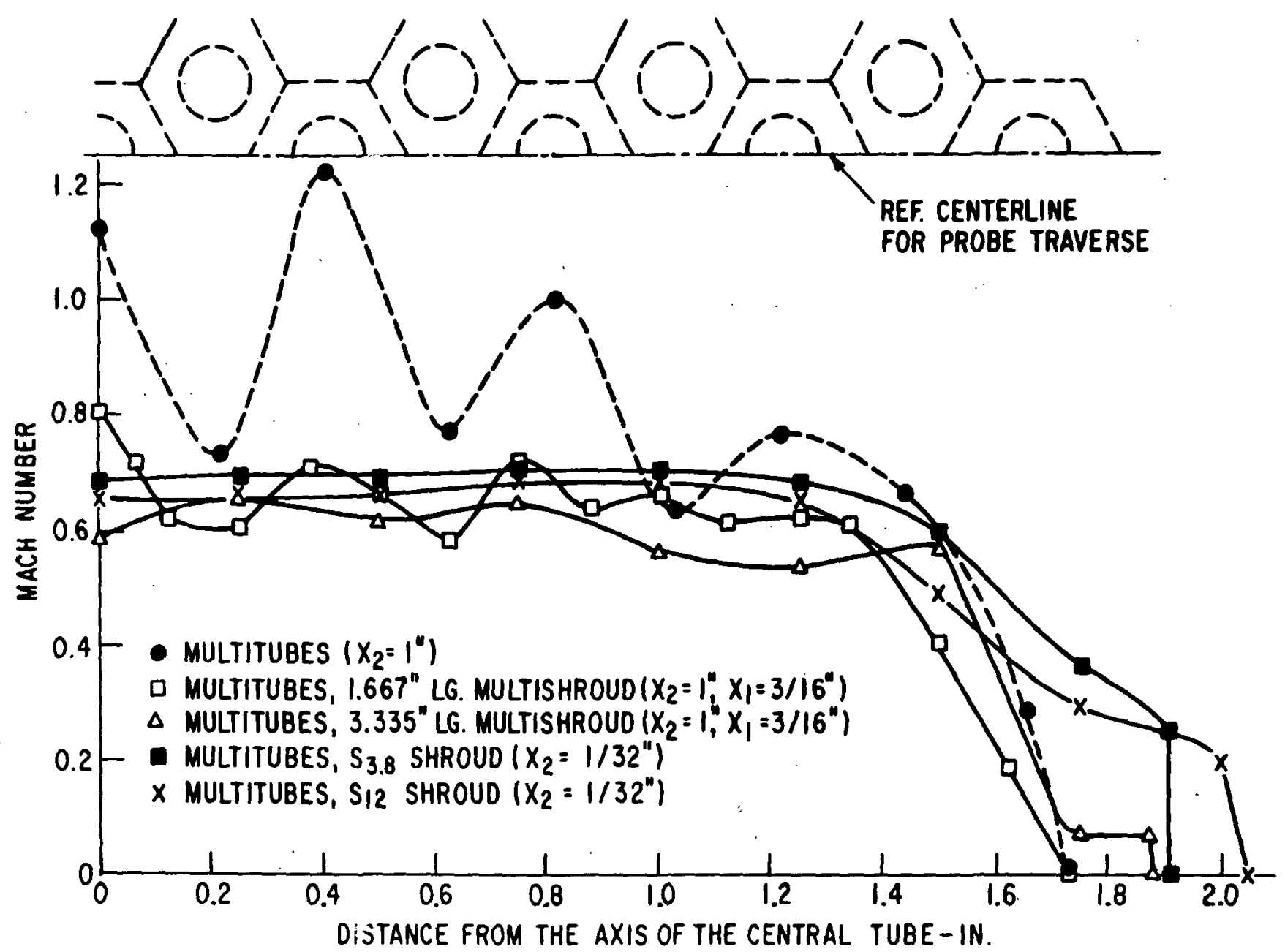

FIG.310 MACH NUMBER PROFILE ACROSS EXITS OF MULTITUBES AND MULTISHROUD 


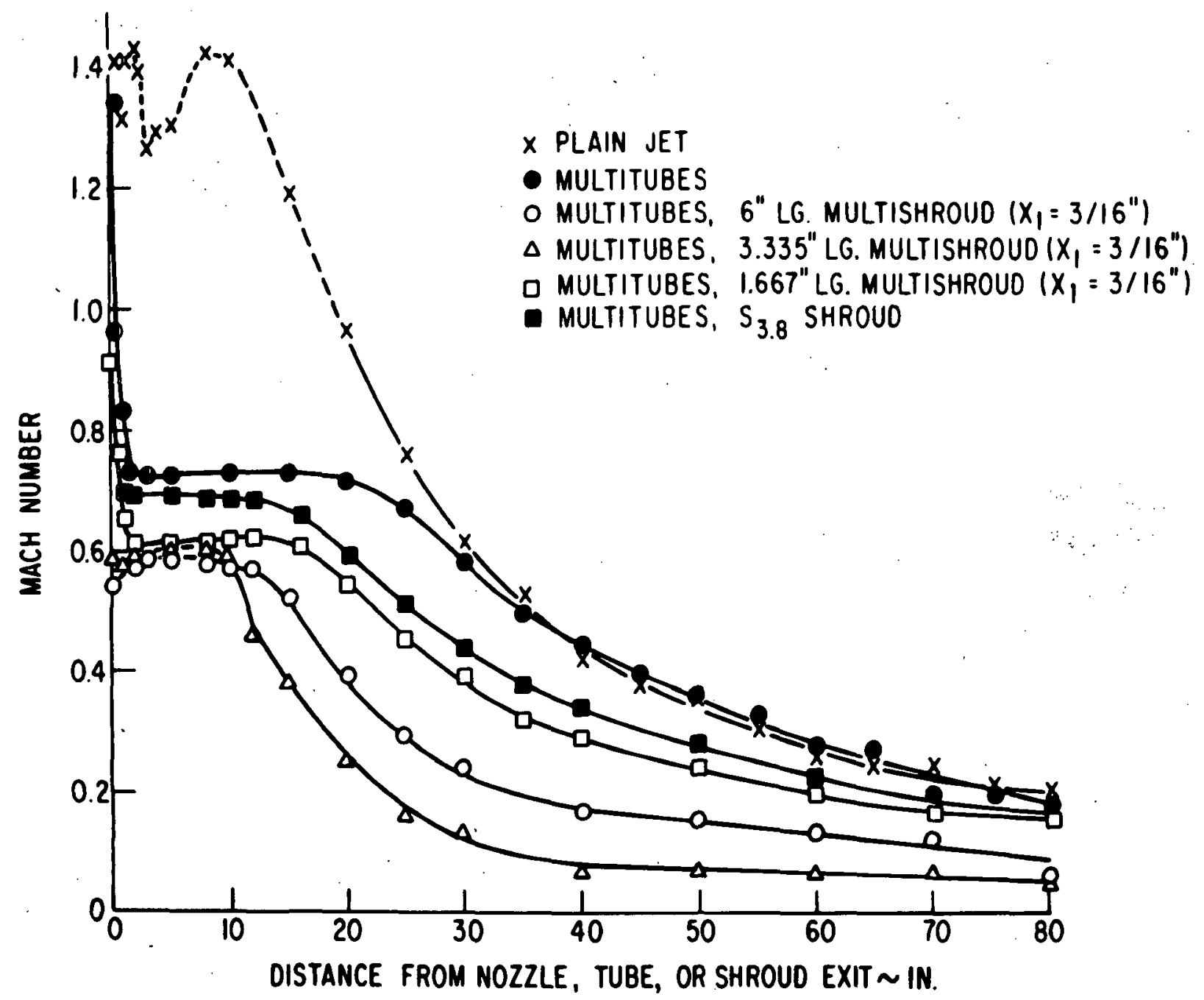

FIG. 3Ib-AXIAL MACH NUMBER VARIATION FOR MULTITUBES WITH ANO WITHOUT SHROUDS 


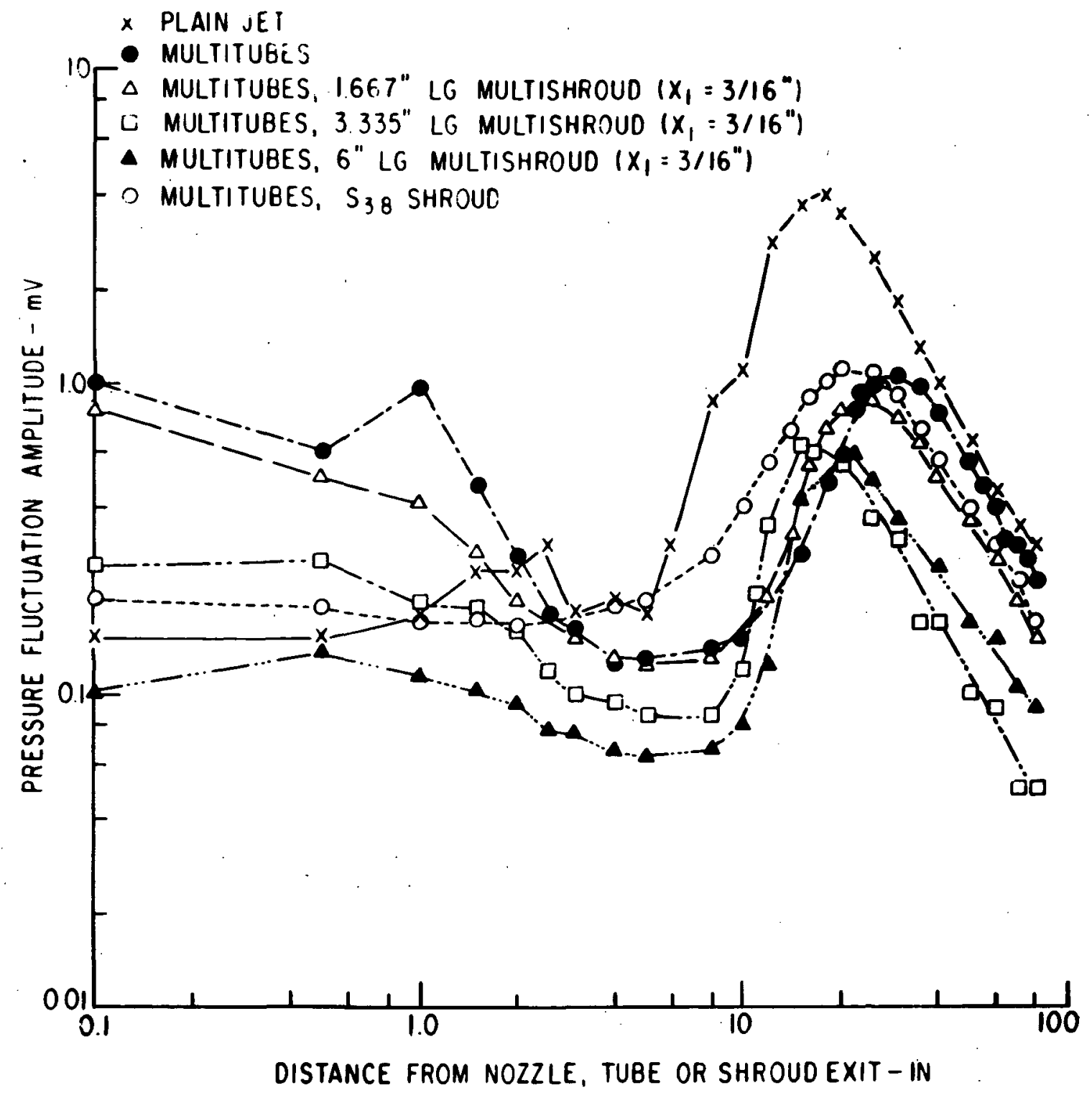

FIG.3IC AXIAL IMPACT PRESSURE FLUCTUATIONS FOR MULTITUBES WITH AND WITHOUT SHROUDS. 


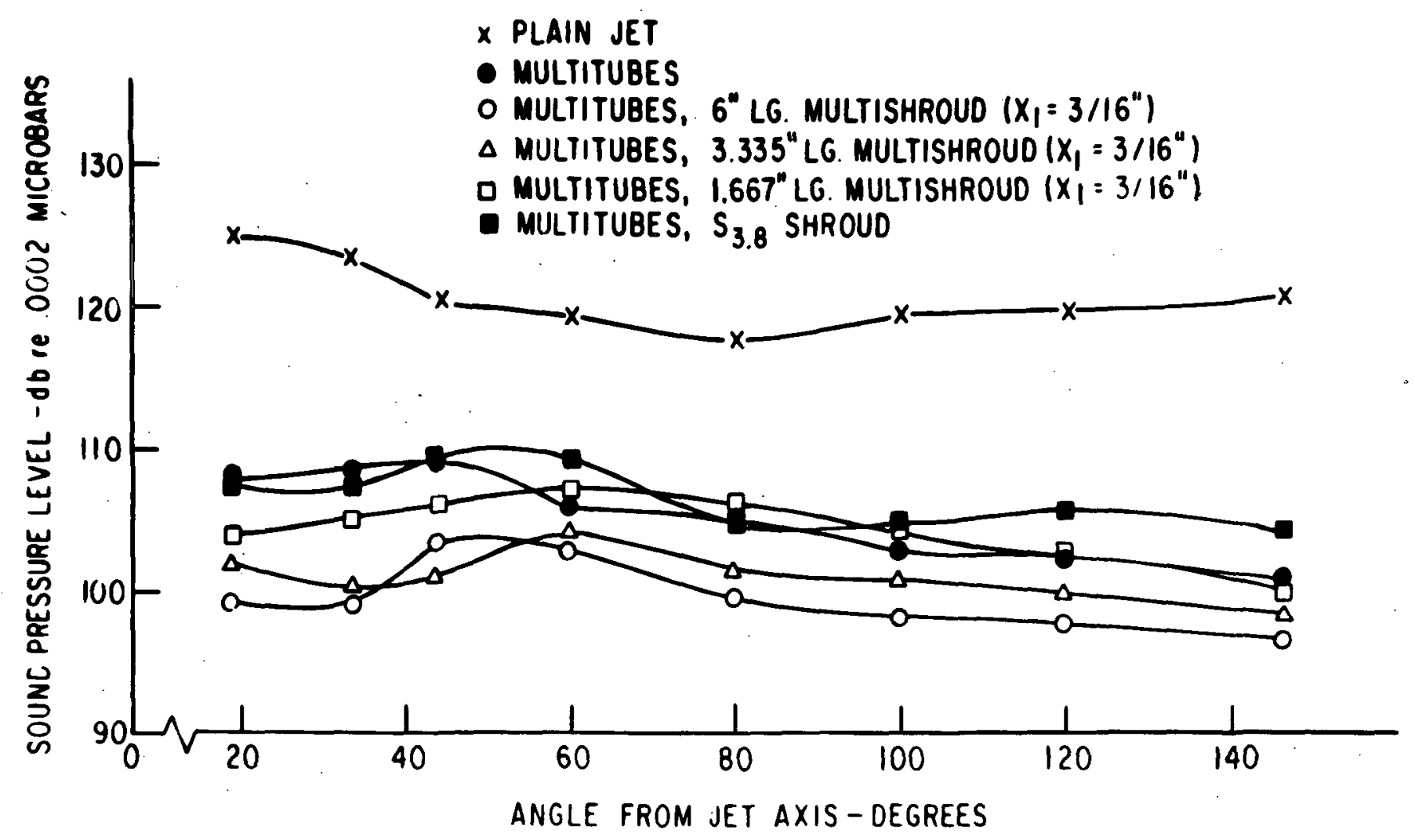

FIG.3Id OVERALL SOUND PRESSURE LEVEL AS A FUNCTION OF ANGULAR POSITION FROM JET AXIS FOR MULTITUBES WITH AND WITHOUT SHROUDS 


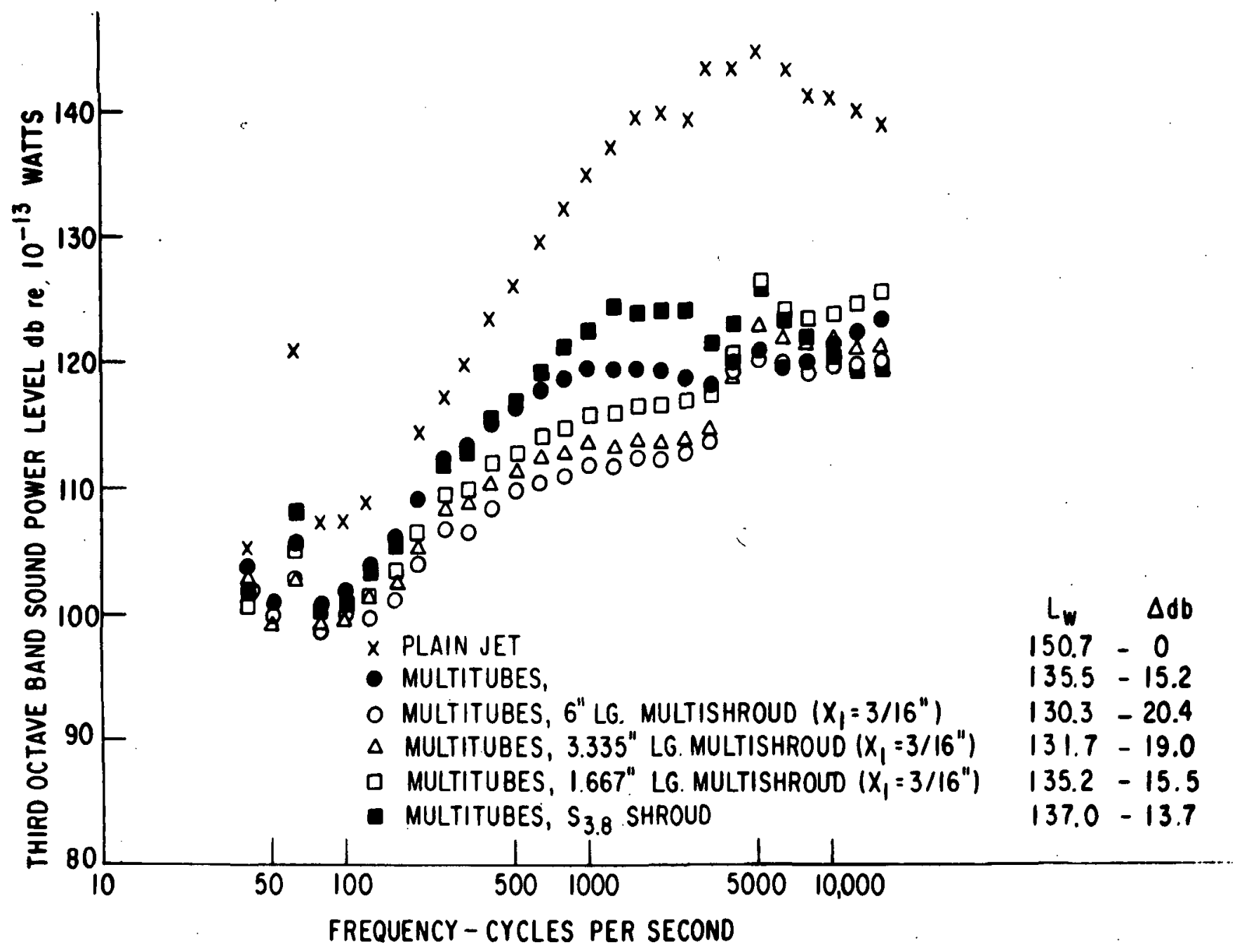

FIG.31 SOUND POWER LEVEL SPECTRA FOR MULTITUBES WITH AND WITHOUT SHROUDS 


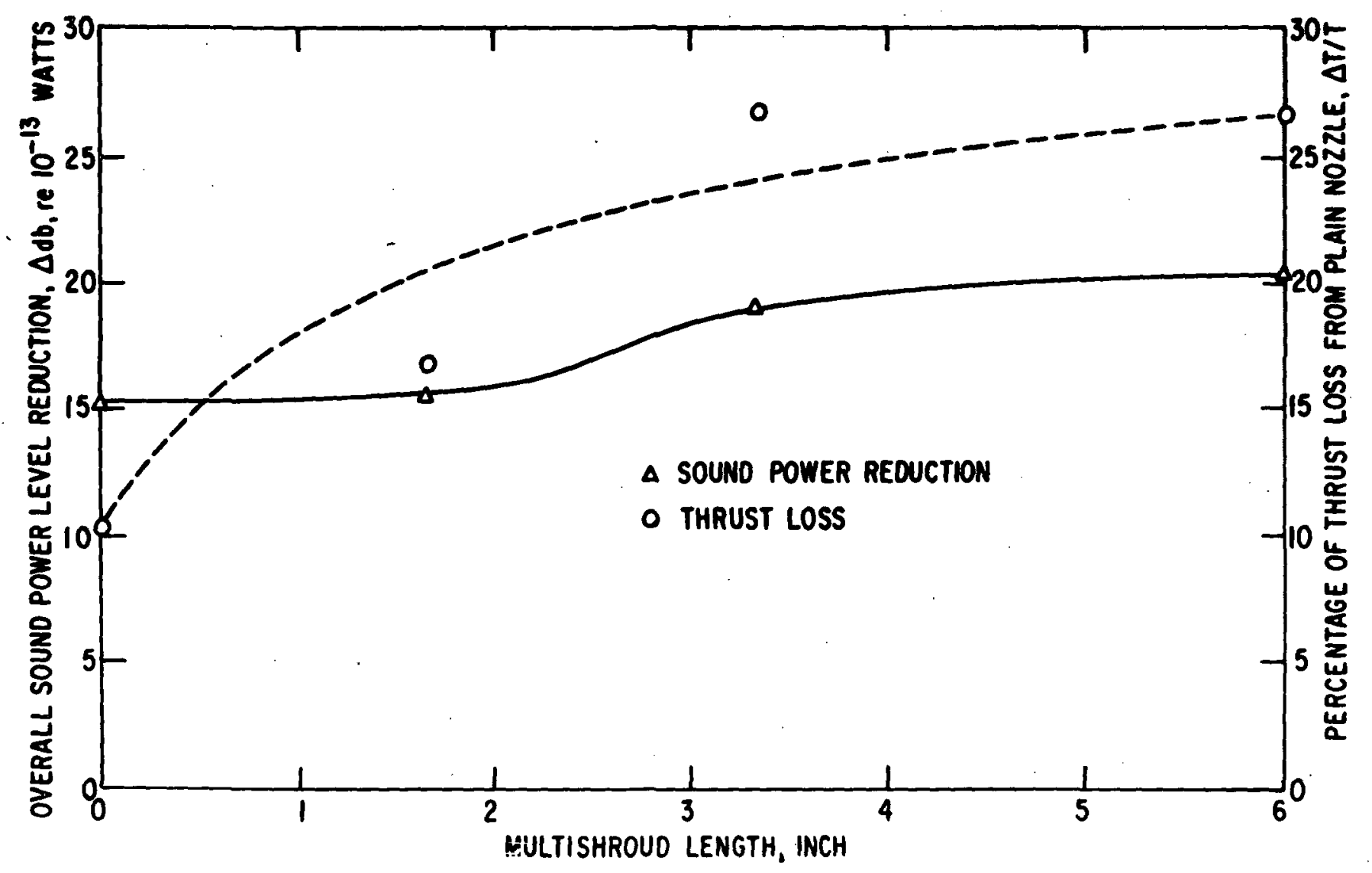

FIG.3If VARIATION OF OVERALL SOUND POWER LEVEL REDUCTION AND PERCENTAGE THRUST LOSS FOR 19| TUBES WITH 191-SHROUD LENGTH 


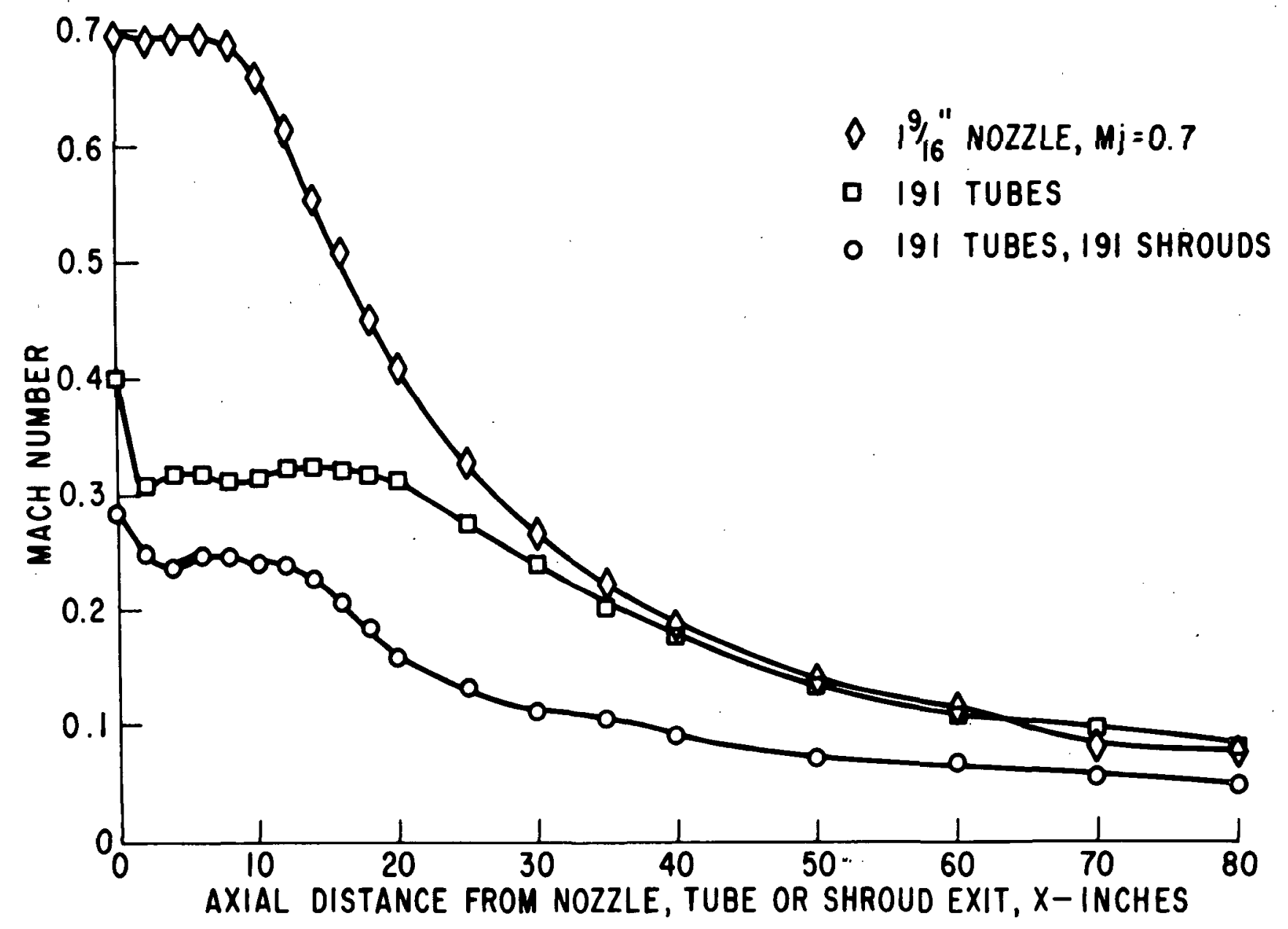

FIG.320 AXIAL MACH NUMBER DISTRIBUTION FOR MULTITUBES WITH AND WITHOUT SHROUDS, $\mathrm{Mj}=0.7$ 


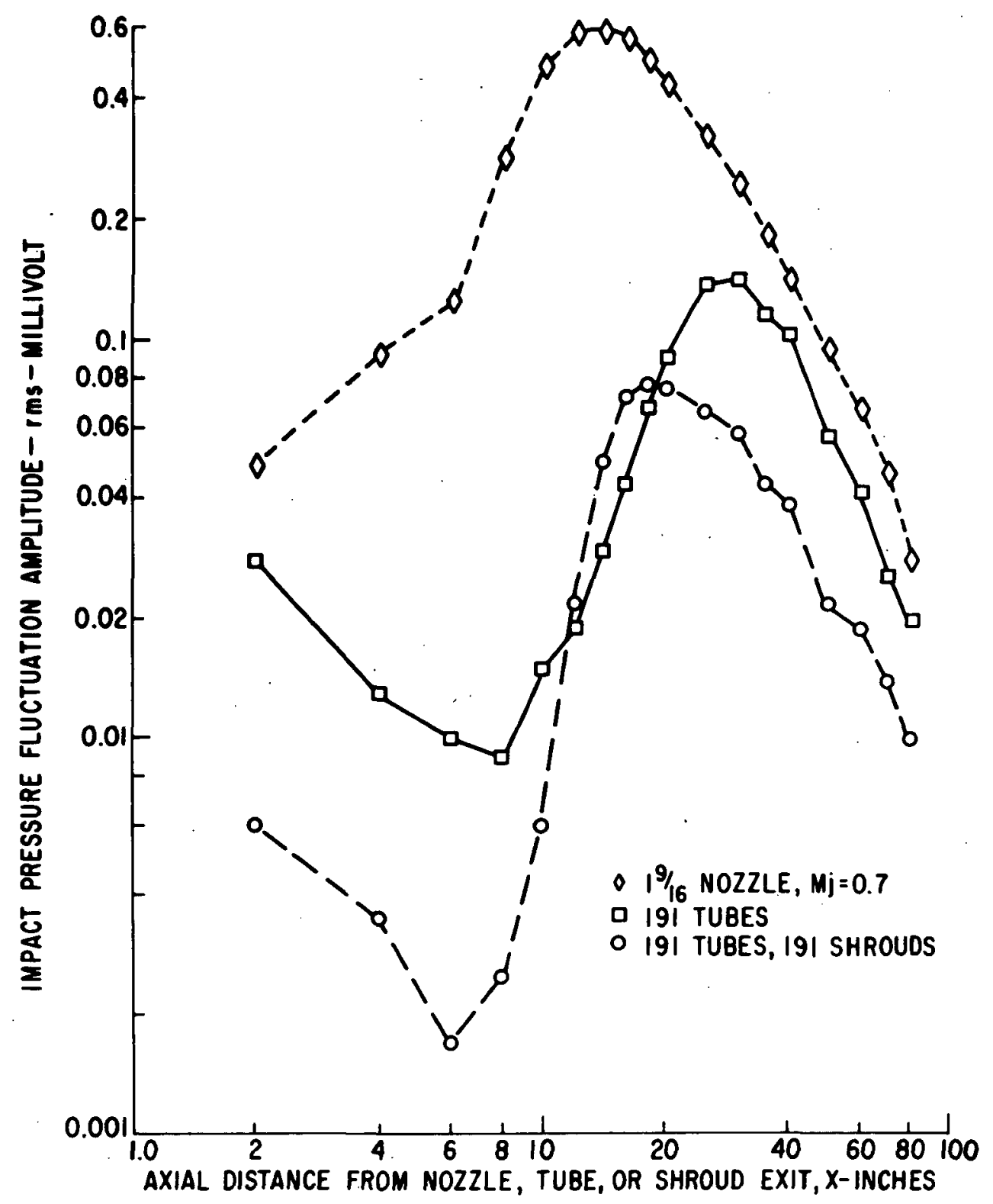

FIG.32b AXIAL IMPACT PRESSURE FLUCTUATIONS FOR MULTITUBES WITH AND WITHOUT SHROUDS, $M j=0.7$ 


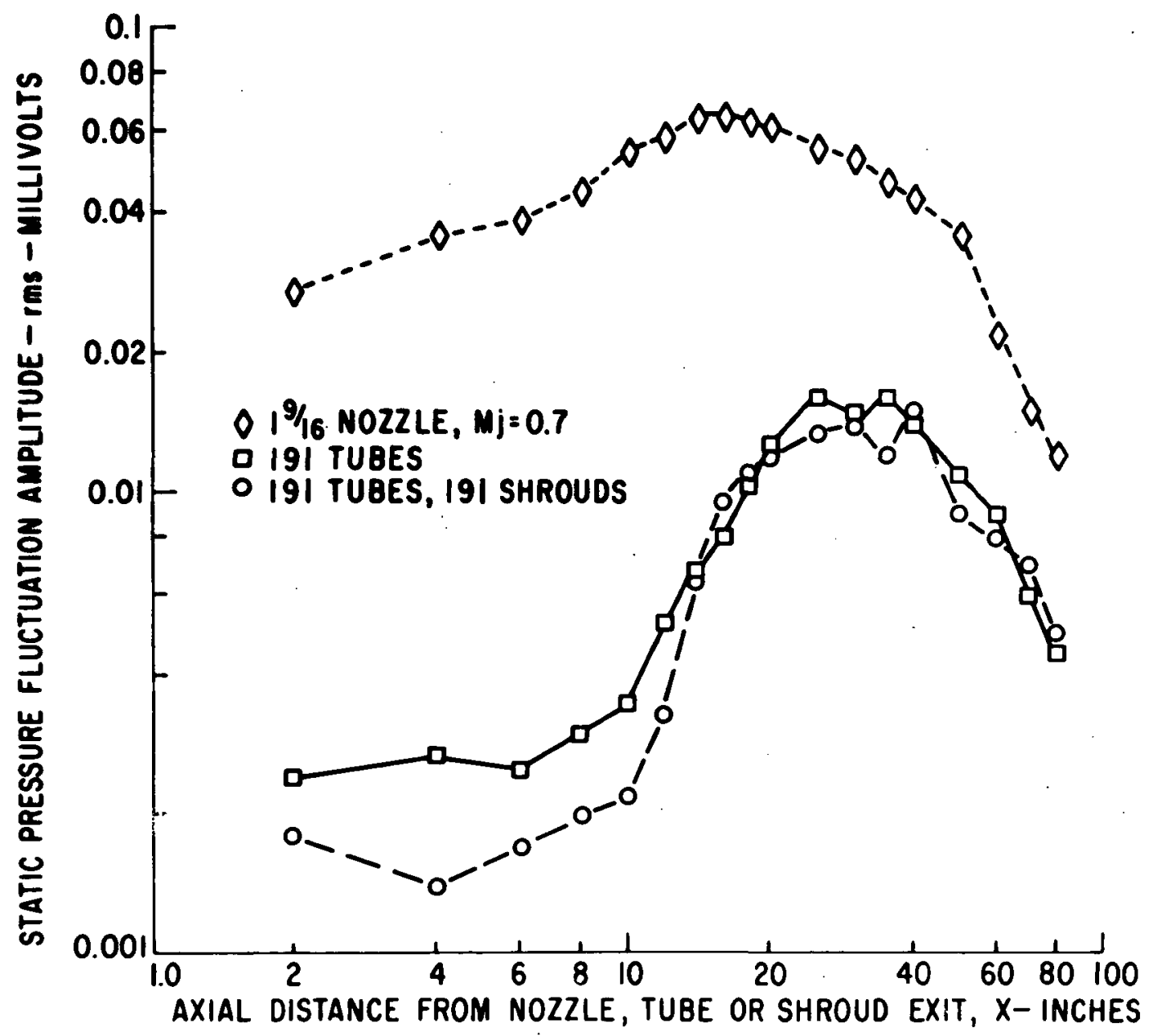

FIG. 32C AXIAL STATIC PRESSURE FLUCTUATIONS FOR MULTITUBES WITH AND WITHOUT SHROUDS, $M j=0.7$ 


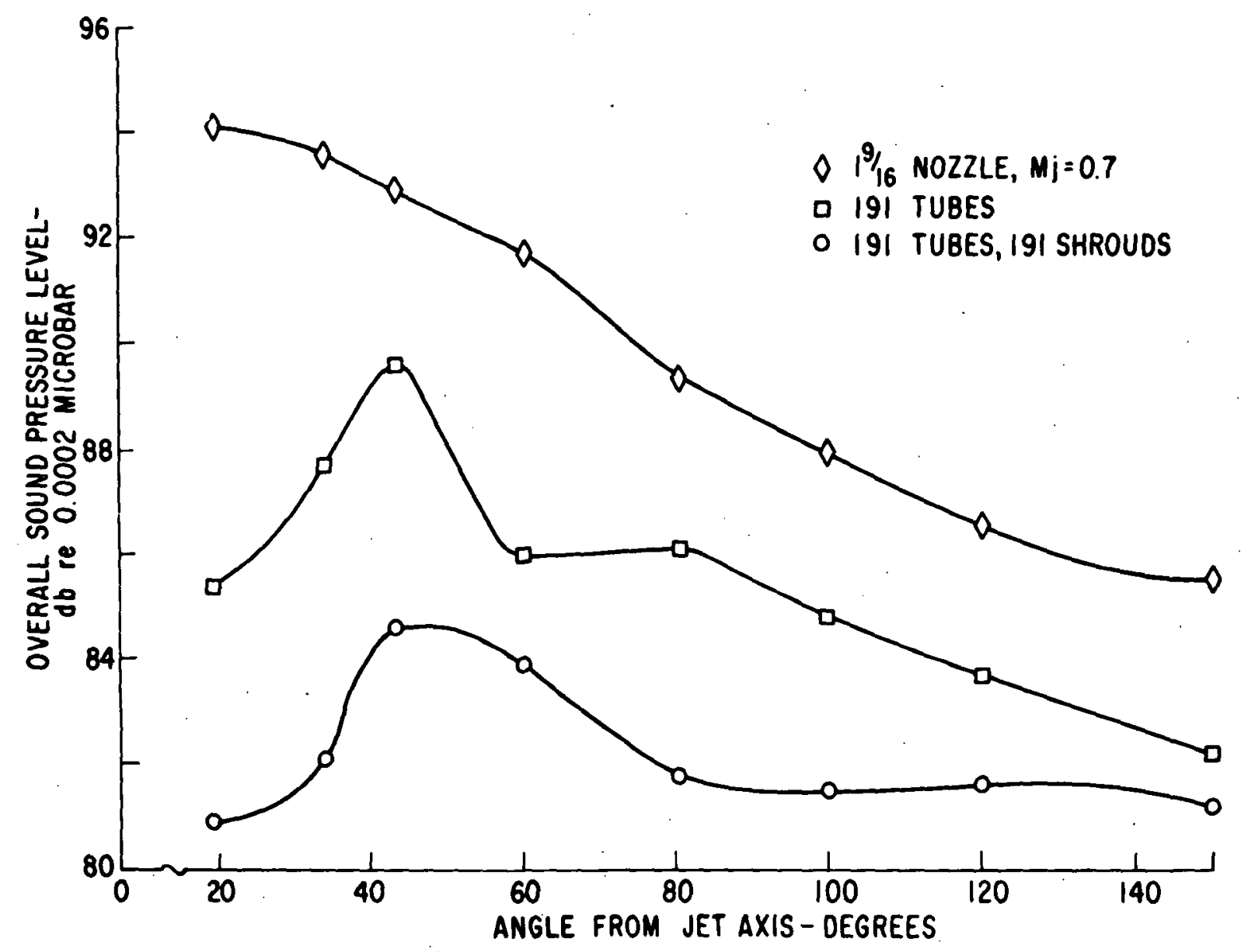

FIG.32d OVERALL SOUND PRESSURE LEVEL AS A FUNCTION OF ANGULAR POSITION FROM JET AXIS FOR MULTITUBES WITH AND WITHOUT SHROUDS, Mj $=0.7$ 


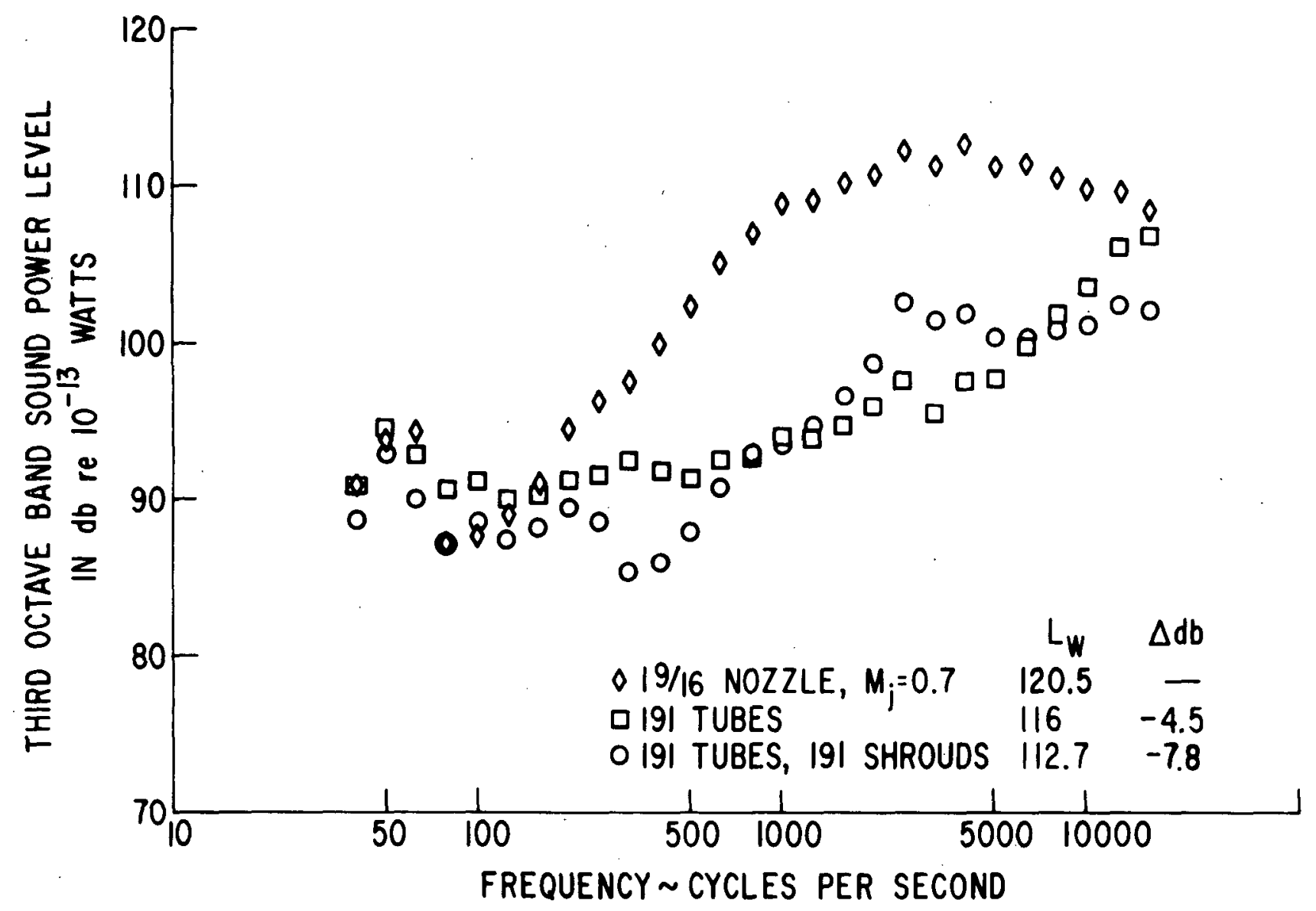

FIG.32e SOUND POWER LEVEL SPECTRA FOR MULTITUBES WITH AND WITHOUT SHROUDS, $M_{j}=0.7$ 